\title{
ACOUstic CHARACTERIStics of MiCROBubbles: EfFeCt OF ACOUSTIC PRESSURE AND PULSE DURATION
}

\author{
by \\ Laxman Subedi \\ Masters of Physics, Tribhuvan University, Nepal, 2006
}

\author{
A thesis \\ presented to Ryerson University \\ in partial fulfillment of the \\ requirements for the degree of \\ Master of Science \\ in the Program of \\ Biomedical Physics
}

Toronto, Ontario, Canada, 2013

(CLaxman Subedi 2013 


\section{AUTHOR'S DECLARATION}

I hereby declare that I am the sole author of this thesis. This is a true copy of the thesis, including any required final revisions, as accepted by my examiners.

I authorize Ryerson University to lend this thesis to other institutions or individuals for the purpose of scholarly research

I further authorize Ryerson University to reproduce this thesis by photocopying or by other means, in total or in part, at the request of other institutions or individuals for the purpose of scholarly research.

I understand that my thesis may be made electronically available to the public. 


\title{
Acoustic characteristics of microbubbles: Effect of acoustic pressure and pulse duration
}

Master of Science 2013

Laxman Subedi

Biomedical Physics

Ryerson University

\begin{abstract}
The acoustic mechanism of ultrasound- Microbubble (MB) mediated sonoporation enhanced permeability of cell membranes has been associated with inertial and stable cavitations. The main objective of this study was to characterise acoustic behaviour of MB, under varying acoustic pressure and pulse duration using passive cavitation detection (PCD) technique. MBs were exposed to $1 \mathrm{MHz}$ ultrasound pulse at varying acoustic pressure (0-1.5 MPa), pulse duration $(5-30 \mu \mathrm{s})$ at pulse repetition frequency of $1 \mathrm{KHz}$ for insonation time of 1 second. The cavitation phenomena of MBs were detected passively using two transducers at center frequencies of 0.5 and $2.25 \mathrm{MHz}$. The MB acoustic response was characterised using FFT algorithm and cavitation dose method. Results indicated that the nonlinear oscillation of MBs increased with PNP and pulse duration. The integrated cavitation dose (ICD) increased with acoustic pressure and decreased with pulse duration. Definity MB showed greater ICD than Artenga MB.
\end{abstract}




\section{Acknowledgements}

I am immensely grateful to my thesis advisor, Dr.Karshafian for his continuous support, encouragement and mentorship for my study and life since the first day I enrolled to Ryerson University. I owe a huge debt of gratitude for taking me on, and giving me freedom to explore and tracking me time to time, toward enjoyable and challenging research. It was a great pleasure to work with him because of providing constructive \& critical responses, being available at need in the lab and on weekends.

I wish to thank Dr.Kolios and Dr.Czarnota my supervisory committee members who gave me a great experience and provided insightful thoughts and discussion. I value their advice and insight for every step of my progress on personal and professional development.

I would also like to thank all the faculty and staff of the Department of Physics at Ryerson University for their help and support.

I am thankful for the work of Adhitya Pandey and Amin Jabari for helping me in Matlab coding and Yevgeniy Davletshin for helping me writing in Latex.

I thank everyone in Ultrasound Therapy Lab (Dr.Karshafian groups), all of whom have been supportive, encouraging, and enjoyable to work with.

Thanks to the Ryerson starting fund for helping this research.

Finally, special thanks to my parents, family members and friends who contributed continuously throughout my academic path. 


\section{Dedication}

To my Family 


\section{Table of Content}

List of Tables $\quad$ ix

List of Figures $x$

$\begin{array}{lll}1 & \text { Introduction } & 1\end{array}$

$1.1 \quad$ Physics of Ultrasound $\ldots \ldots \ldots$

1.2 Ultrasound Contrast agents $\ldots \ldots \ldots \ldots$

1.3 Applications . . . . . . . . . . . . . . . . . . . . . . 4

1.3 .1 Sonoporation $\ldots \ldots \ldots \ldots \ldots \ldots$

1.4 MB Acoustic Behavior and Quantification . . . . . . . . . . . . 5

1.4 .1 Microbubble acoustic behaviour $\ldots \ldots \ldots \ldots \ldots$

$1.4 .2 \quad$ Effect of acoustic parameters on MB behaviour $\ldots \ldots \ldots$

1.4.3 MBs characterization: Passive Cavitation Detection (PCD) . . . . 11

1.4 .4 Microbubble Signals $\ldots \ldots \ldots \ldots$

1.4 .5 Cavitation Dose . . . . . . . . . . . . . . . . . . 13

1.5 Thesis outline $\ldots \ldots \ldots \ldots \ldots$

\begin{tabular}{lll}
\hline 2 & Materials and Methods & 17
\end{tabular}

$2.1 \quad$ Experimental setup and procedures $\ldots \ldots \ldots \ldots \ldots$ 
2.1.1 Ultrasound Exposure Condition and Passive cavitation detection . 17

$2.1 .2 \quad$ Exposure protocol $\ldots \ldots \ldots \ldots$

2.1 .3 Data Acquisition . . . . . . . . . . . . . . . . . . 21

2.1 .4 Transducer Specifications . . . . . . . . . . . . . . . . . . . . . . 22

2.1 .5 Transducer calibration . . . . . . . . . . . . . . . . . . 22

2.2 Microbubble agent $\ldots \ldots \ldots \ldots$. . . . . . . . . . . . . . . . 22

2.3 Experiments performed $\ldots \ldots \ldots \ldots$. . . . . . . . . . . . . 24

2.4 Data Analysis . . . . . . . . . . . . . . . . . . . . . . 25

2.4 .1 Microbubble behaviour . . . . . . . . . . . . . 25

2.4 .2 Cavitation dose . . . . . . . . . . . . . . . . 26

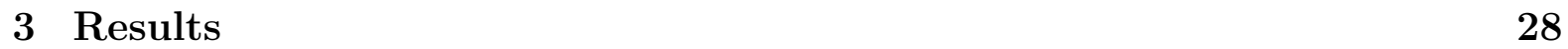

3.1 MB acoustic behaviour . . . . . . . . . . . . . . . . . . . . . . . . . 28

$3.1 .1 \quad$ Effect of acoustic pressure $(\mathrm{PNP})$. . . . . . . . . . . . . . . 28

3.1 .2 Effect of acoustic pressure and pulse duration on MB type . . . . 34

3.2 Cavitation Dose . . . . . . . . . . . . . . . . . . . . . . . . . 38

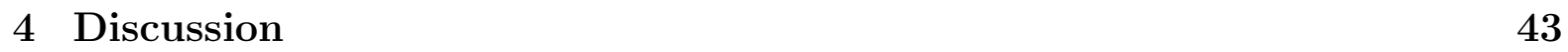

4.1 Nonlinear Oscillation . . . . . . . . . . . . . . . . . . . . . . . 43

4.2 Cavitation dose . . . . . . . . . . . . . . . . . . . 46

4.3 Limitations of this study . . . . . . . . . . . . . . . . . . . . 47

4.4 Possible applications and clinical relevance . . . . . . . . . . . . . . . . 48

5 Conclusion and Future work 44

5.1 Conclusion $\ldots \ldots \ldots \ldots$. . . . . . . . . . . . . . . . . . . 49

5.2 Future work: Effect of Microbubbles Size . . . . . . . . . . . . . . . . . . 49 
5.2 .1 Results and discussion $\ldots \ldots \ldots \ldots$

\begin{tabular}{lr}
\hline Appendices & 54
\end{tabular}

\begin{tabular}{ll}
\hline A Time domain signal & 55
\end{tabular}

\begin{tabular}{ll}
\hline B Experiments Results & 58
\end{tabular}

\begin{tabular}{lr}
\hline References & 72
\end{tabular} 


\section{List of Tables}

1.1 Table for Ostwald coefficient and disapperance time . . . . . . . . . . . . 3

$2.1 \quad$ List of PCD experiments: where $\mathrm{PD}=$ pulse duration, $\mathrm{PNP}=$ Peak negative pressure and FIL/UNF represents filtered/unfiltered bubbles and NOE represents numbers of experiments. . . . . . . . . . . . . . . . 25 


\section{List of Figures}

$1.1 \quad$ Ultrasound microbubble induced sonoporation phenomenon. . . . . . . . 5

1.2 Microbubble Behaviours in an Ultrasound Field. . . . . . . . . . . . . . . . . 7

1.3 Cavitation mechanism inducing bio-effects. . . . . . . . . . . . . . . 9

1.4 MBs signals, including subharmonics, second harmonic, ultraharmobnics

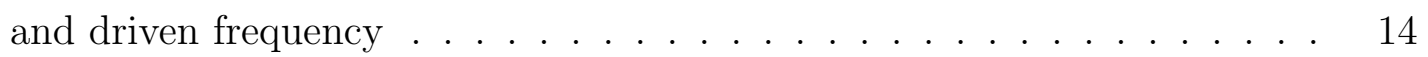

$2.1 \quad$ Schematic diagram of experimental Passive cavitation detection system. . 18

2.2 Transducers alignment at the focus of mylair membraned tube . . . . . . 19

2.3 Diagram showing the exposure pulse sequence and data acquisition timing.

PD : pulse duration (5 and $30 \mu \mathrm{s})$, PRP : pulse repetition period (1ms),

TD : trigger delay $(60 \mu \mathrm{s})$ and AW : acquisition window $(48 \mu \mathrm{s})$. . . . . . 21

$2.4 \quad 1 \mathrm{MHz}$ transducer calibration curve for pressure measurements . . . . . 23

2.5 Volume weighted size distribution measurements with Coulter Counter for Artenga MBs . . . . . . . . . . . . . . . . . . . . . 24

2.6 Data analysis algorithm . . . . . . . . . . . . . . . . . . . . . 27

$3.1 \quad$ TD signals and their corresponding FFT spectra for ART MB at acoustic pressure of 0-1.5 MPa, 30 cycle pulse duration and PCD signals received with $2.25 \mathrm{MHz}$ transducer. . . . . . . . . . . . . . . . . . . . . . . . . . 29 
3.2 TD signals and their corresponding FFT spectra for ART MBs at acoustic pressure of $0.5-1.5 \mathrm{MPa}, 30$ cycle pulse duration and PCD signals received with $0.5 \mathrm{MHz}$ transducer. . . . . . . . . . . . . . . . . . . . . . . . . . . 30

3.3 Spectrograms as a function of PNP for signals received with 0.5 and 2.25 $\mathrm{MHz}$ receiver transducers. . . . . . . . . . . . . . . . . . . . . 32

$3.4 \quad$ FFT spectra at various time points, for the data received with $2.25 \mathrm{MHz}$

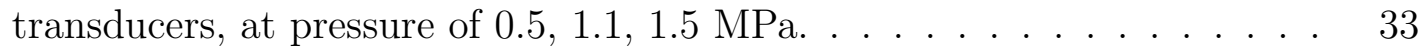

3.5 FFT spectra for ART and DEF MBs, at 5 and 30 cycle pulse duration, and PNP $(0.5,1.1,1.5 \mathrm{MPa})$ for signals received with $2.25 \mathrm{MHz}$ transducer 34

3.6 Spectrograms for ART MB, at 5 cycles pulse duration and PNP $(0.5,1.1$, 1.5) MPa for the signals received with $2.25 \mathrm{MHz}$ transducer . . . . . . . 36

3.7 Spectrograms for DEF MB, at 5 and 30 cycles pulse duration and PNP ( $0.5,1.1,1.5) \mathrm{MPa}$ for the signals received with $2.25 \mathrm{MHz}$ transducer . . 37

3.8 Cavitation dose plots for ART MB at 30 cycles pulse duration at pressure of $0.5,1.1$ and $1.5 \mathrm{MPa}$. . . . . . . . . . . . . . . . . . . . . . . . . . . . 39

3.9 Integrated cavitation dose for the subharmonic frequency spectrum for DEF and ART MBs at acoustic pressure of $0.5,1.1$ and $1.5 \mathrm{MPa}$ and 30

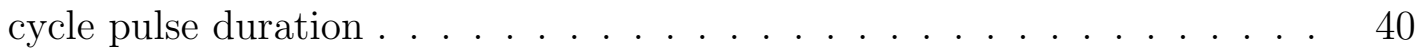

3.10 Integrated cavitation dose for the ultraharmonic and second harmonic frequency spectra for DEF and ART MBs at acoustic pressure of 0.5, 1.1 and $1.5 \mathrm{MPa}$ and 30 cycle pulse duration . . . . . . . . . . . . . . . . . . 41

3.11 Integrated cavitation dose for the subharmonic frequency spectrum for DEF and ART a MBs at acoustic pressure of 0.5, 1.1 and $1.5 \mathrm{MPa}$ and 5 cycle pulse duration . . . . . . . . . . . . . . . . . . . . . . . . 42 
3.12 Integrated cavitation dose for the ultraharmonic and second harmonic frequency spectra for DEF and ART MBs at acoustic pressure of 0.5, 1.1 and $1.5 \mathrm{MPa}$ and 5 cycle pulse duration . . . . . . . . . . . . . . . . . . . . . 42

$5.1 \quad$ Spectrograms for filtered DEF MBs, at 30 cycle pulse duration and PNP $(0.5,1.1,1.5 \mathrm{MPa})$ for the signals received with $2.25 \mathrm{MHz}$ transducer . . 51

5.2 Spectrograms for filtered DEF MBs, at 30 cycle pulse duration and PNP $(0.5,1.1,1.5 \mathrm{MPa})$ for the signals received with $0.5 \mathrm{MHz}$ transducer . . 52

$5.3 \quad$ Integrated cavitation dose for filtered DEF MBs at the subharmonic,ultraharmonics and second harmonic frequency spectra at acoustic pressures of of $0.5,1.1$ and $1.5 \mathrm{MPa} . \ldots \ldots . \ldots . \ldots . \ldots 53$

A.1 Portion of TD signal for Figure 3.1 at $0.5,1.1,1.5 \mathrm{MPa}$. . . . . . . . . . 56

A.2 Portion of TD signal for Figure 3.2 at $0.5,1.1,1.5 \mathrm{MPa}$. . . . . . . . . . 57

B.1 Spectrograms for ART MB as a function of PNP for signals received with $2.25 \mathrm{MHz}$ receiver transducer. . . . . . . . . . . . . . . . . . . . . . 59

B.2 [Spectrograms for DEF MB as a function of PNP for signals received with $2.25 \mathrm{MHz}$ receiver transducer. . . . . . . . . . . . . . . . . . . 59

B.3 Spectrograms for ART MB as a function of PNP for signals received with $2.25 \mathrm{MHz}$ receiver transducer. . . . . . . . . . . . . . . . . . . . . . 60

B.4 Spectrograms for DEF MB as a function of PNP for signals received with $2.25 \mathrm{MHz}$ receiver transducer. . . . . . . . . . . . . . . . . . . . 60

B.5 Spectrograms for ART and DEF MBs as a function of PNP for signals received with $0.5 \mathrm{MHz}$ receiver transducer. . . . . . . . . . . . . . . . . . 61 
B.6 FFT spectra for ART and DEF MBs as a function of PNP for signals received with $2.25 \mathrm{MHz}$ receiver transducers. . . . . . . . . . . . . . . . . 61

B.7 Cavitation dose plots for DEF MB at 30 cycles pulse duration at pressure of $0.5,1.1$ and $1.5 \mathrm{MPa}$. . . . . . . . . . . . . . . . . . . . . . . . . . . . . 62

B.8 Cavitation dose plots for ART MB at 30 cycles pulse duration at pressure

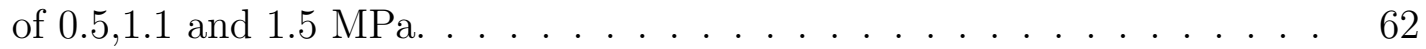

B.9 Cavitation dose plots for DEF MB at 5 cycles pulse duration at pressure

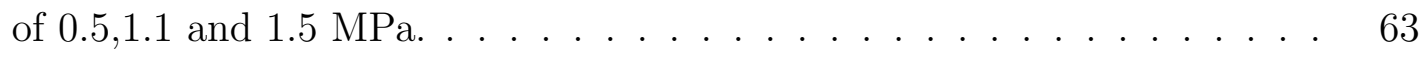

B.10 Cavitation dose plots for ART MB at 30 cycles pulse duration at pressure of $0.5,1.1$ and $1.5 \mathrm{MPa}$. . . . . . . . . . . . . . . . . . . . 63 


\section{List of Abbreviations}

ACD

ART

DEF

FDA

FFT

HMDEF

HMART

ICD

MB

PCD

PNP

PRF

RMS

RA

SHART

SHDEF

TD

UCAs

$\mathrm{UH}$

UHART

UHDEF
Active cavitation detection.

Artenga.

Definity.

Food and drug administration.

Fast Fourier Transfer.

Harmonic for Definity.

Harmonic for Artenga.

Integrated cavitation dose.

Microbubble.

Passive cavitation detection.

Peak negative pressure.

Pulse repitition frequency.

Root mean square.

Receiver amplifier.

Subharmonic for Artenga.

Subharmonic for Definity.

Time domain signal.

Ultrasound contrast agents.

Ultraharmonic.

Ultraharmonic for Artenga

Ultraharmonic for Definity. 


\section{Chapter 1}

\section{Introduction}

\subsection{Physics of Ultrasound}

Ultrasound, sound at frequencies greater than $20 \mathrm{KHz}$, is a mechanical wave that propagates through a medium by the oscillation of its molecules or particles, transferring energy from one to another. In fluids and biological tissues, the oscillation of the particles is parallel to the direction of wave propagation where the propagating disturbances follow the form of pressure (longitudinal) waves with regions of compression (high density) and rarefaction (low density) (1). During propagation, the pressure waves interact with the underlying tissue resulting in scattering and absorption (2). Scattering of the ultrasound wave occurs when the pressure wave encounters regions of different acoustic impedances. The acoustic impedance of a material for a plane wave is given by

$$
Z=\rho c
$$

Where $\rho$ is the density of the medium and $c$ is the speed of sound. The speed of 
sound in liquids is given as

$$
c=\sqrt{\frac{\beta}{\rho}}
$$

where $\left(\beta=\rho \frac{d p}{d \rho}\right)$ is the bulk modulus of the medium.

Moreover the scattering of ultrasound waves from a small spherical scatterer of diameter $a \ll \lambda$ at the far field depends on local variations in density and compressibility (3), given by equation

$$
p_{s}(r, \Theta)=p_{i} e^{-j k r} \frac{k^{2}}{R^{3}} 3 r\left[\frac{\kappa_{\nu}-\kappa_{0}}{\kappa_{0}}+\frac{3\left(\rho_{\nu}-\rho_{0}\right)}{2 \rho_{\nu}+\rho_{0}} \cos \Theta\right]
$$

where $p_{s}$ is the scattered peak pressure, $p_{i}$ is the incident pressure amplitude, $k=2 \pi f / c$ is the acoustic wave number and $R=a / 2$ is the radius of the scatterer. $\Theta$ represents the angle between the incident wave and the radiated sound in dipole fashion and $\kappa$ and $\rho$ represent compressibility and density of the medium. The subscripts o and $\nu$ represents properties of the scatterer and surrounding medium respectively. The fractional change in compressibility given by the first term of the equation is the (monopole) term, which is the result of a pulsating point source while the difference in densities of the medium in the second term contribute scatterer to undergo back and forth motion, called as dipole term. In the case of air the scattering is mainly dominated by the compressibility term and thus air/gas-filled microbubbles have important applications as ultrasound contrast agents. 


\section{$1.2 \quad$ Ultrasound Contrast agents}

Ultrasound contrast agents are comprised of shell-encapsulated gas-filled micron-sized (less than $10 \mu \mathrm{m}$ ) bubbles, which are administered intravenously for imaging applications. From the clinical perspective microbubbles (MBs) have to be biocompatible, capable of crossing the pulmonary capillary bed after injection, and be stable enough to achieve contrast enhancement for the duration of ultrasound imaging. The first generation of MBs were air-filled bubbles without encapsulation which do not persist long enough to be of practical use in the human body. An improvement was made using higher molecular weight gases in a core and encapsulation with the shell. Gases such as perfluorocarbon, nitrogen or sulfur hexafluoride, have a lower Ostwald coefficient (the dimensionless ratio of solubility of the gas in liquid to gas density) and higher molecular weights which diffuse at a slower rate compared to those having a higher Ostwald's coeefficient (4) as shown in Table 1.1.

Table 1.1: Ostwald coefficient and disappearance time (time for diffusion of gas when microbubble is in free gas state) for $3 \mu \mathrm{m}$ diameter bubbles having different gases.

\begin{tabular}{|l|c|c|}
\hline gases & Ostwald's coefficient $\left(10^{6}\right)$ & Disapperance time $(s)$ \\
\hline Air & 23,168 & 0.02 \\
\hline Sulfur hexafluoride $\left(S F_{6}\right)$ & 5,950 & 0.1 \\
\hline Perfluropropane $\left(C_{3} F_{8}\right)$ & 538 & 1.1 \\
\hline Perflurohexane $\left(C_{6} H_{14}\right)$ & 24 & 2 \\
\hline
\end{tabular}

The encapsulation (shell) of the MB is comprised of protein, lipid or polymer layer. The shell affects the acoustic response of the MB, for example, the coating dampens the vibrations of the MBs and changes the microbubble resonance frequency (discussed in section 1.4). 


\subsection{Applications}

MBs were originally developed to enhance the ultrasound scattered signals from blood vessels and the heart chamber. MBs are used clinically to improve imaging of cancerous tissues by detecting the tumor vasculature (5). In therapeutic applications, MBs can enhance the passage of bioactive materials across blood vessels (6), opening the blood brain barrier (BBB) to enhance local delivery of drugs to glioma (7). Moreover, MBs are used for the potential uptake of materials (drugs/genes) into cells or across biological membranes through transient opening of pores called sonoporation (8). In addition, MBs can be loaded with drug and genetic materials (9) and act as a vector of bioactive compounds. MBs can be tagged with ligands on their surface, and bind to specific disease sites in the body (10) that enhance targeted delivery.

\subsubsection{Sonoporation}

Sonoporation is a transient, reversible and nonlethal alteration of cell membrane permeability by ultrasound-MB mediated approach (11). This allows possible transportation of biologically actiive molecules such as DNA (12) chemotherapeutic drugs (13) genetic materials $(14)$ and proteins delivery $(15)$ to cells and tissues. Thus, sonoporation- mediated drug and gene therapy is currently being developed for cancer, and blood-brain-barrier limited treatments $(16,7)$. Figure 1.1 replicated from (11) illustrates how ultrasound and MB exposure enhance delivery of molecules across the cell membrane.

The MBs acoustic behaviour associated with sonoporation includes MB oscillation and disruption in an ultrasound field. Stable cavitation including linear and nonlinear oscillations of microbubbles $(17 ; 18)$ and inertial cavitation $(19)$ which is results of MBs disruption phenomenons have been shown to enhance cell permeability. Expanded 


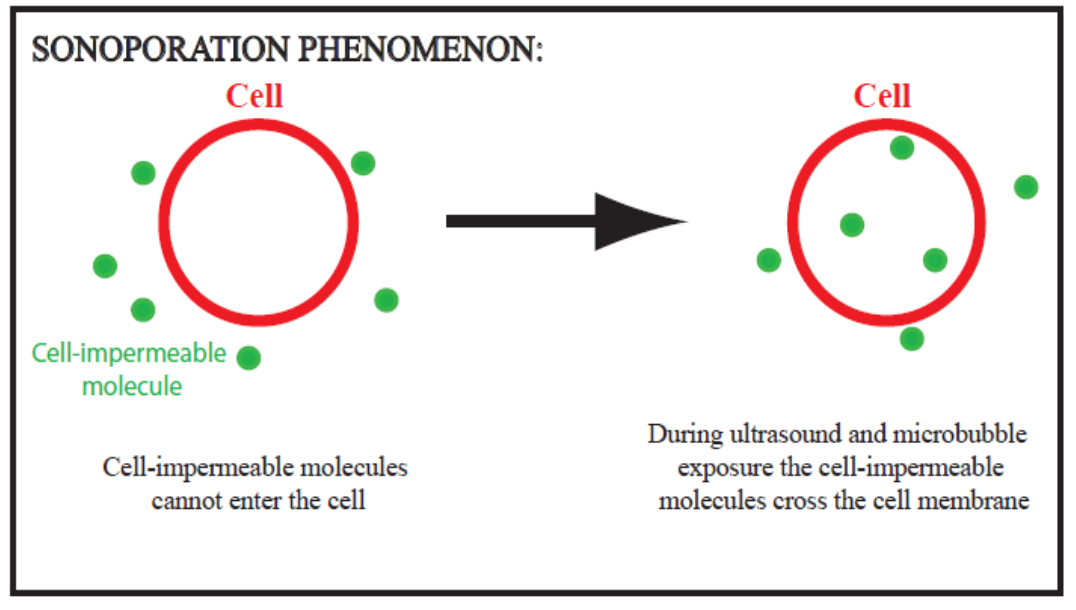

Figure 1.1: Sonoporation phenomenon: The application of ultrasound on MB increases the permeability of cell membranes and allows delivery of molecules into the cell.

knowledge of $\mathrm{MB}$ response may allow us to understand the biophysical mechanisms of snoporation and to improve and potentially control ultrasound-MB mediated therapeutic application.

\subsection{MB Acoustic Behavior and Quantification}

\subsubsection{Microbubble acoustic behaviour}

The acoustic behavior of the MBs depend on ultrasound exposure parameters such as acoustic pressure, frequency, pulse duration and Pulse repition frequency; and physical properties of microbubble such as size, concentration, gas and shell composition; and the surrounding environment (20). In the presence of the time-varying pressure of the US wave, MBs undergo a series of phenomena including formation, expansion or growth by rectified diffusion, and destruction of MBs called as acoustic cavitation (21). This process involves the conversion of low pressure of the ultrasound field into high pressure within 
small MBs (22). This acoustic energy within the MBs causes it to oscillate, expand and finally dissolve or collapse.

Cavitation commonly includes two types of bubble activities: namely, inertial and stable cavitations (Figure 1.2). Stable cavitation refers to prolonged volumetric shape oscillations of a MB, that can persist for several cycles in an ultrasound field (22). At lower acoustic pressure, the microbubble undergoes linear oscillation which is described by the linear oscillator model. The resonance frequency, $\omega_{0}$, of a free gas bubble, assuming a linear and an undamped system, (neglecting surface tension and damping) is given by

$$
\omega_{0}=\frac{1}{2 \pi R_{0}} \sqrt{\frac{3 \kappa_{0} P_{0}}{\rho}}
$$

where $\kappa_{0}$ represents the ratio of heat capacities at constant pressure to volume, $R_{0}$ is equilibrium radius of the $\mathrm{MB}, \rho$ is the density of the medium and $P_{0}$ is the static pressure of the surrounding medium at the bubble surface.

Equation (1.4) shows that the MB experiences a resonance that depends on the size of the bubble. It is believed when a bubble is driven at this resonant frequency, it reaches the highest oscillation amplitude and may provide more effective therapeutic results. In addition, for the case of encapsulated MB the shell increases the stiffness and viscous damping which dampens the motion of MB wall. This decrease the scattering ability of $\mathrm{MB}$ and thus increase the resonance frequency. At high acoustic pressures the microbubbles undergo nonlinear oscillation, which is described by modified Rayleigh Plessettee-type equation. Bubbles undergoing stable cavitation scatter the incident ultrasound beam with energy content at the driven frequency $(f)$, during linear oscillations (expansion and contraction of MB by same amount) and harmonics (nf), subharmonics $(f / 2)$ or ultraharmonics $(\mathrm{m} f / \mathrm{n})$ during nonlinear oscillation (asymmetric expansion and 


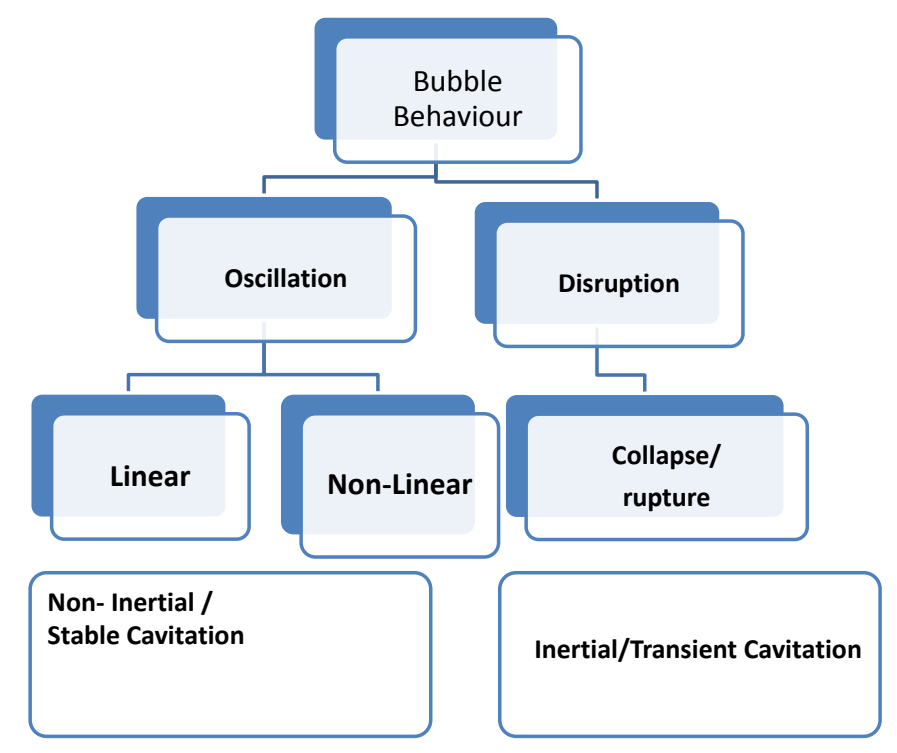

Figure 1.2: Microbubble Behaviours in an Ultrasound Field.

contraction) (23). This nonlinear oscillation has been explored for imaging applications. In addition MB oscillations in this regime produces circulating eddies (microstreaming) in the surrounding area of bubbles (24) with velocities and shear proportional to amplitude of oscillation, which is believed to cause stress on cell membranes and increase the permeability that enhance sonoporation.

Inertial cavitation is the rapid expansion (numbers of times the initial radius) and violent collapse (25) of the gas bubble that is caused at the higher acoustic pressure due to inertial effect of the surrounding liquid. It occurs for microseconds in few acoustic cycles which has limitations on the temporal resolution. Several mechanism of MB destructions including fragmentation into smaller bubbles, gradual gas diffusion and shell breakage are described previously. Destruction of MB is determined by coating of MBs. For rigid shelled MBs such as Quantison and BP127 the destruction is accompanied by shell rupture and gas release while soft shelled MBs such as Definity, Optison and Sonovue 
the disruption may be associated with violent collapse. MB undergoing this phenomenon scatters the acoustic energy in the form of broadband emissions related to post excitation signals (26). Moreover when the microbubble undergoes inertial collapse, this results in microjets $(27)$ when it is close to the boundary that can puncture tissue and cell membranes, shockwaves (28) during implosion that produces stress on biological materials and free radical production have its application in sonoluminiscence. Since MBs collapse occurred suddenly with the large amount of acoustic energy released it is believed to cause lethal damage to biological tissues.

Beside these activities, At certain conditions of ultrasound exposures, the phospolipid coated microbubble exhibits complex phenomena such as buckling and ruptures $(29)$ and compression only behaviours (30).

Stable and inertial cavitation mechanisms enhanced by MB activity play important roles in several ultrasound-based therapeutic applications such as shockwave lithotripsy to fragment kidney stones (31), thrombolysis (32; 33), tissue ablation (34), disruption of the blood brain barrier (BBB ) (16) and targeted drug/gene delivery $(35 ; 36 ; 37)$. In addition, cavitation has been studied for its significant potential in intracellular drug/gene delivery through the sonoporation technique, a medically significant approach that deliver the drugs/genes within the cells of the tissue in an efficient, spatially specific and safe way $(8 ; 38 ; 17)$. Figure 1.3 replicated from $(22)$ shows the different cavitation activities inducing bio-effects.

\subsubsection{Effect of acoustic parameters on MB behaviour}

As mentioned above the acoustic response of a MB depends on a number of factors, including its physical properties (e.g. size, gas and shell), ultrasound exposure parameters, 


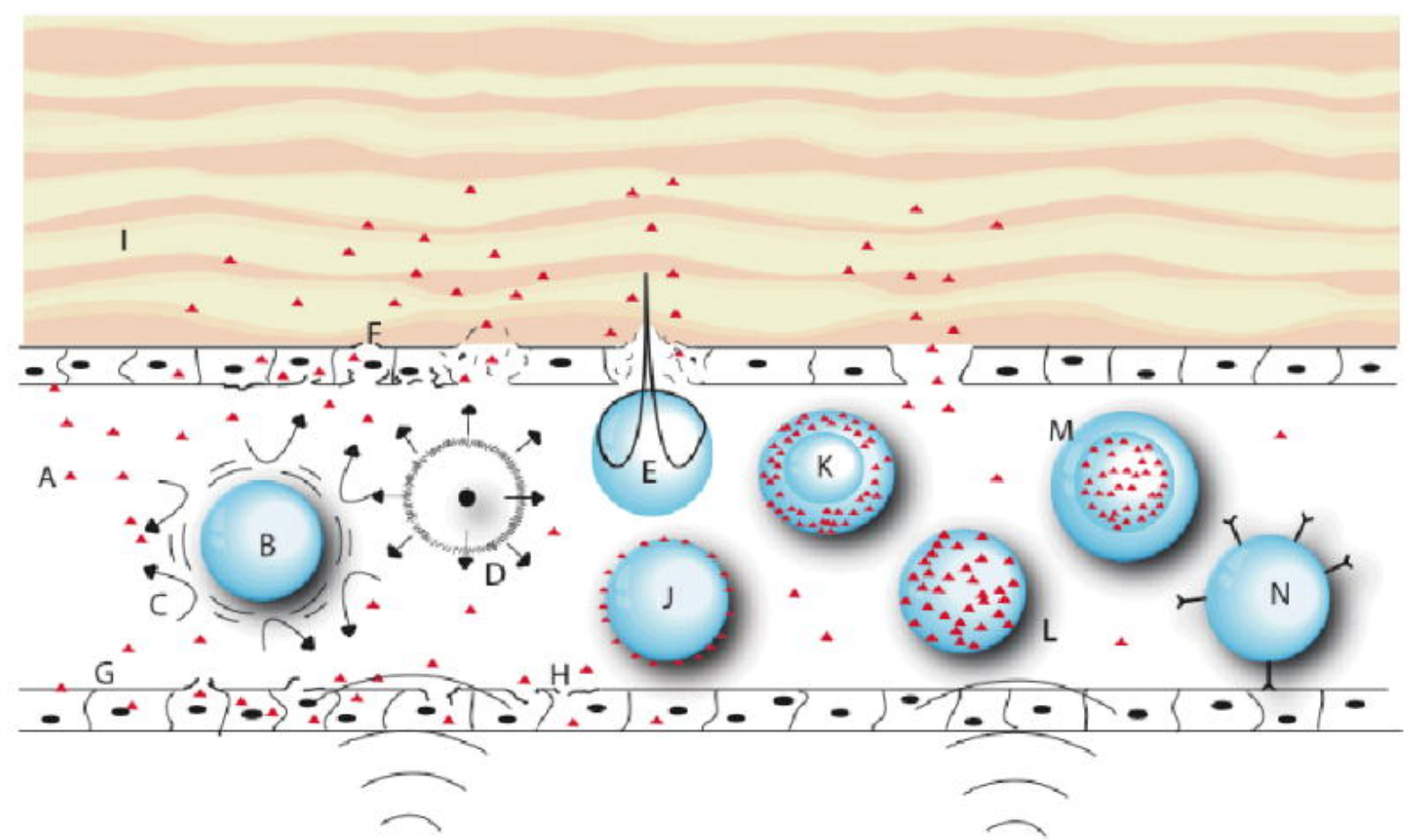

Figure 1.3: illustrate the cavitation mechanism inducing bio-effects.(A: therapeutic agent (triangles); B: gas bubble undergoing stable cavitation; C: microstreaming around cavitating bubble; D. collapse cavitation emitting a shock wave; E: asymmetrical bubble collapse producing a liquid jet that pierces the endothelial lining; F: completely pierced and ruptured cell; G: non-ruptured cells with increased membrane permeability due to insonation; $\mathrm{H}$ : cell with damaged membrane from microstreaming or shock wave; I: extravascular tissue; J: thin-walled microbubble decorated with agent on surface; K. thickwalled microbubble with agent in lipophilic phase; L: micelle with agent in lipophilic phase; M: liposome with agent in aqueous interior; $\mathrm{N}$ : vesicle decorated with targeting moieties attached to a specific target).

and environmental conditions (boundary condition, ambient pressure and temperature) (39; 40). Experiments have been carried out with varying acoustic pressure, frequency and pulse durations using acoustic methods. Experiments providing nonlinear oscillations of Optison MBs insonified at 2 and $4 \mathrm{MHz}$ have shown that the first and second harmonic components increase linearly with acoustic pressure and the subharmonic and ultraharmonic components undergo rapid growth in the intermediate acoustic pressure 
range (1.5MPa) (41). The MB acoustic behaviors of Optison and Definity were studied at peak to peak pressures (1.6, 2.0 and 2.4 MPa) with $2.8 \mathrm{MHz}$ centre frequency transducer (42). It was reported that with increase of pressure the spectral magnitude increases for $f / 2, f / 4$ and for broadband spectra. The rupture threshold for Optison were studied at centre frequencies of $0.9,2.8$ and $4.6 \mathrm{MHz}$, pulse durations of $3,5,7$ cycles of center frequency and peak negative pressures of 0.07 to $5.39 \mathrm{MPa}$. It was found that the rupture threshold increased with frequency and decreased with pulse duration (26). The stable and transient subharmonic emissions for Sonovue MBs, using 30 cycle pulse duration at 3.3 and $5 \mathrm{MHz}$ and acoustic pressures of $40-225 \mathrm{kPa}$, was studied and have shown that emissions time of the stable and transient subharmonic thresholds were different (43). The subharmonic emission of 5 different agents at high frequency $(25 \mathrm{MHz})$ have been investigated and have shown that with increase of pulse duration the subharmonic emissions are enhanced for all agents (44). The subharmonic emissions for Sonovue MB was studied at 3 and 30 cycle pulse duration and have shown that the emissions were higher at longer pulse duration of 30 cycles (45). Beside these the time dynamics of MBs at these varying acoustic conditions hasn't been investigated which is the focus of this thesis.

All of these finding motivate a rigorous investigation on the dependency of MB behavior on acoustic pressure and pulse duration. This may provide an understanding of MBs nonlinear oscillation with respect to time at exposure parameters which has important implication in inducing bioeffects. 


\subsubsection{MBs characterization: Passive Cavitation Detection (PCD)}

Experimentally MBs have been characterized through acoustic emissions $(25 ; 46)$ and optical imaging at high frame rates $(47,48 ; 30)$. Both of these techniques are employed for understanding the oscillation and disruption behaviour of the MBs. The acoustic method involves active cavitation detection (ACD) and passive cavitation detection (PCD) for sensing MBs behaviours. The ACD method is based on the pulse-echo technique, which measures the MB emissions generally with a single transducer. Since focus is the same for pulse and echo detection, with the same transducer, this method has greater spatial and temporal resolution (49). In PCD, the transducers or hydrophones are operated in receiving mode only, where the receiver measures the emissions from MB $(49 ; 50 ; 51$; 52). In this study we measure the bubble emissions using the passive cavitation detection (PCD) technique.

\subsubsection{Microbubble Signals}

Analysis of PCD signals measured from MBs is commonly performed in the frequency domain which exhibits characteristic emissions from microbubbles including the driving frequency, harmonics, subharmonics, ultraharmonics and broadband emissions. The information from the frequency spectrum helps to understand the cavitation phenomenon which is associated with the MB oscillation and disruption. The main characteristics of the frequency spectrum includes:

\section{Fundamental or driven frequency $(f)$}

At low stable cavitation regime when the MB is at rest or undergoing small oscillations, it emits the frequency spectrum of having frequency equal to that of driving frequency called the fundamental frequency. Usually this spectrum can be observed 
with or without MBs (water/tissue). As this signal is emitted from both tissue and $\mathrm{MB}$, it is not useful on characterizing the MB behaviour.

2. Second harmonic $(2 f)$

As the acoustic pressure amplitude increases, the MB oscillates nonlinearly emitting second harmonic (frequency spectrum having frequency twice that of driving frequency) of higher magnitude compared to that of tissue. This spectrum is associated with nonlinear propogation of ultrasound and has been exploited for harmonic imaging applications for the contrast enhancement (53). For therapeutic applications second harmonic emissions has to be explored for its usefulness.

3. Higher harmonics $(\mathrm{n} f)$

At higher acoustic pressures, the MBs signals exhibit higher harmonics at $3 f, 4 f$ and so on for transmitted frequency $f(4)$. The exact cause of these higher harmonics are not well understood, however it has been proposed that the nonlinear oscillation of the bubbles leading to chaotic behavior are associated with emission of harmonics (23).

4. Subharmonic $(f / 2)$ and ultraharmonics $(\mathrm{m} f / 2)$

Oscillating bubbles have potential to emit subharmonic peaks at integer fractions of driving frequency e.g $f / 2$ and ultraharmonic peaks at $(\mathrm{m} f / 2)$ where $\mathrm{m}$ is $3,5 \ldots$ for transmitted frequency $f$, which are not possible in tissue. These unique markers of the MB characteristics are associated with bubble dynamics. Investigations on sub-harmonics imaging $(54 ; 44)$ and in therapy 445$)$ have shown that subharmonic emission can be exploited as indicator of sonoporation. Although the mechanism behind subharmonic-ultraharmonic emissions are not well understood, four possi- 
ble explanation have been suggested for free bubbles: (i) transient cavitation, (ii) subharmonic oscillation of bubbles excited at resonance, (iii) resonant oscillations of bubbles excited at their second harmonic and (iv) non-radial modes of oscillation. These phenomena of subharmonic and ultraharmonics emissions are described using bifurcation diagram (23) where bubble undergoes period doubling, period of four and eight. For better understanding detail investigation on sub-harmonics detection and its application has to be explored.

\section{Broadband signal}

Further increase of the pressure amplitude will cause the MBs to increase its size more than two times its initial radius and finally collapse or rupture. This phenomenon of bubble collapse occurs within a very short period of time where broadband spectra are emitted from MBs which are associated with post excitation signals $(26,55)$. In therapeutic applications this has been studied for inducing bioeffects $(56 ; 57 ; 39)$. Figure 1.4 replicated from $(58)$ represents the FFT response of MBs extract in frequency domain.

\subsubsection{Cavitation Dose}

Bioeffects induced by ultrasonically-stimulated MB have been associated with cavitation dose (57; 56; 39). Cavitation dose represents the amount of acoustic energy that is contained in the desired frequency window and is obtained by plotting the RMS FFT amplitude vs time (56). Furthermore the cumulative/integrated cavitation dose can be obtained from the integration of the entire RMS FFT amplitude vs time curve over the exposure time (decribe detail in section 2.4.2). In this project we estimate the cavitation dose and integrated cavitation dose using equations (2.2) and (2.3). 


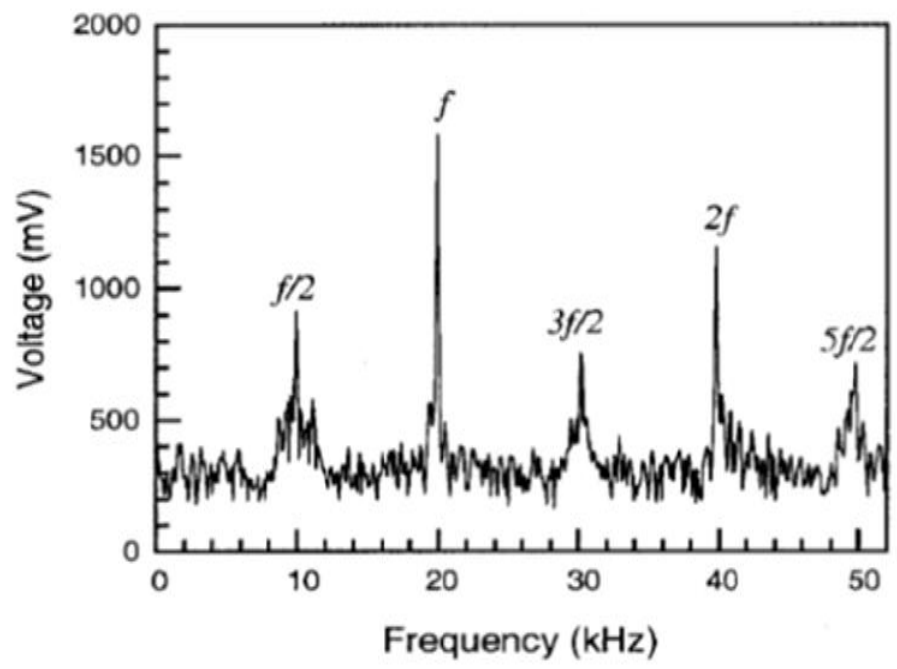

Figure 1.4: Figure: Showing the frequency spectra: fundamental $(f)$, subharmonic $(f / 2)$ ultraharmonics $(3 f / 2,5 f / 2)$ and second harmonic $(2 f)$ from MBs measured with acoustic method.

Numbers of studies were performed in vitro (39) and in vivo (59;60) conditions. The inertial cavitation dose as a function of acoustic pressure, MBs concentration, and pulse length on hemolysis (vitro) and endothelial cell damage (vivo) have been studied. The conclusion was that both hemolysis and endothelial damage incrseases with cavitation dose. Similarly correlation of inertial cavitations as a function of applied pressure, bubble concentration and pulse duration with sonoporation have been investigated by (57; 56) where they have shown the cavitation dose correlates with pore sizes and sonoporation. All these experiments were performed with broad size distribution of MBs which strongly affects their emissions. Moreover, these experiments were performed under different exposure conditions and thus variation in cavitation dose and their resulting bio-effects was observed. Thus rigorous investigation on the MB acoustic behaviors controlling the exposure parameters and corresponding cavitation dose have to be optimized/controlled for producing useful bio-effects to improve sonoporation applications. Therefore the mo- 
tivation behind the study is that the understanding of acoustic response of MB and their quantification at various acoustic pressure and pulse duration may potentially improve and monitor the ultrasound-MB mediated sonoporation applications.

\subsection{Thesis outline}

The hypothesis guiding this research is that the acoustic pressure and pulse duration have significant effect on MB acoustic behaviour and cavitation dose.

The main objective of this thesis is to characterize the acoustic behaviour of MBs at varying exposure conditions using the passive cavitation detection method.

The specific objectives of this study are:

- To measure the acoustic emission from MBs using passive cavitation detection at various pressures and pulse durations and study their response through FFT spectral analysis.

- To investigate the effect of ultrasound pressure and pulse duration on different MBs (Artenga and Definity) acoustic response.

- To quantify these bubble response through cavitation dose method.

The long term goal is to develop a monitoring tool of MBs activities during ultrasound treatment based on the PCD method, with aim to improve applications of drug /gene delivery using ultrasound.

Ultrasound and MB, their applications, MB response and their quantification and an outline of thesis is discussed in first chapter. In the second chapter, the PCD experimental setup and protocal, data acquisition and their analysis is discussed. Results are shown 
for MB behaviours and cavitation dose, as a function of acoustic pressure and pulse duration in third chapter. The fourth chapter contains a discussion, limitation of study and possible clinical relevance. The fifth chapter includes conclusions and directions for future work, with results for effect of MB sizes on their acoustic behaviours and appendix includes additional data to support results in chapter 3. 


\section{Chapter 2}

\section{Materials and Methods}

PCD experiments were conducted with MBs (Artenga and Definity) in suspensions exposed to ultrasound at various acoustic pressure and pulse duration. The MBs response were analyzed in terms of frequency contents, and their quantification was performed through cavitation dose methods.

\subsection{Experimental setup and procedures}

\subsubsection{Ultrasound Exposure Condition and Passive cavitation detection}

A schematic diagram of the experimental setup for passive cavitation measurement is shown in Figure (2.1).

It consist of a programmable function generator (WW5062 Tabor Electronic Ltd, NY, USA) that transmits the short sequenced trigger controlled sine pulses (5 and 30 cycles)

for insonation time of $1 \mathrm{~s}$ at a constant pulse repetition frequency (PRF) of $1 \mathrm{KHz}$. 


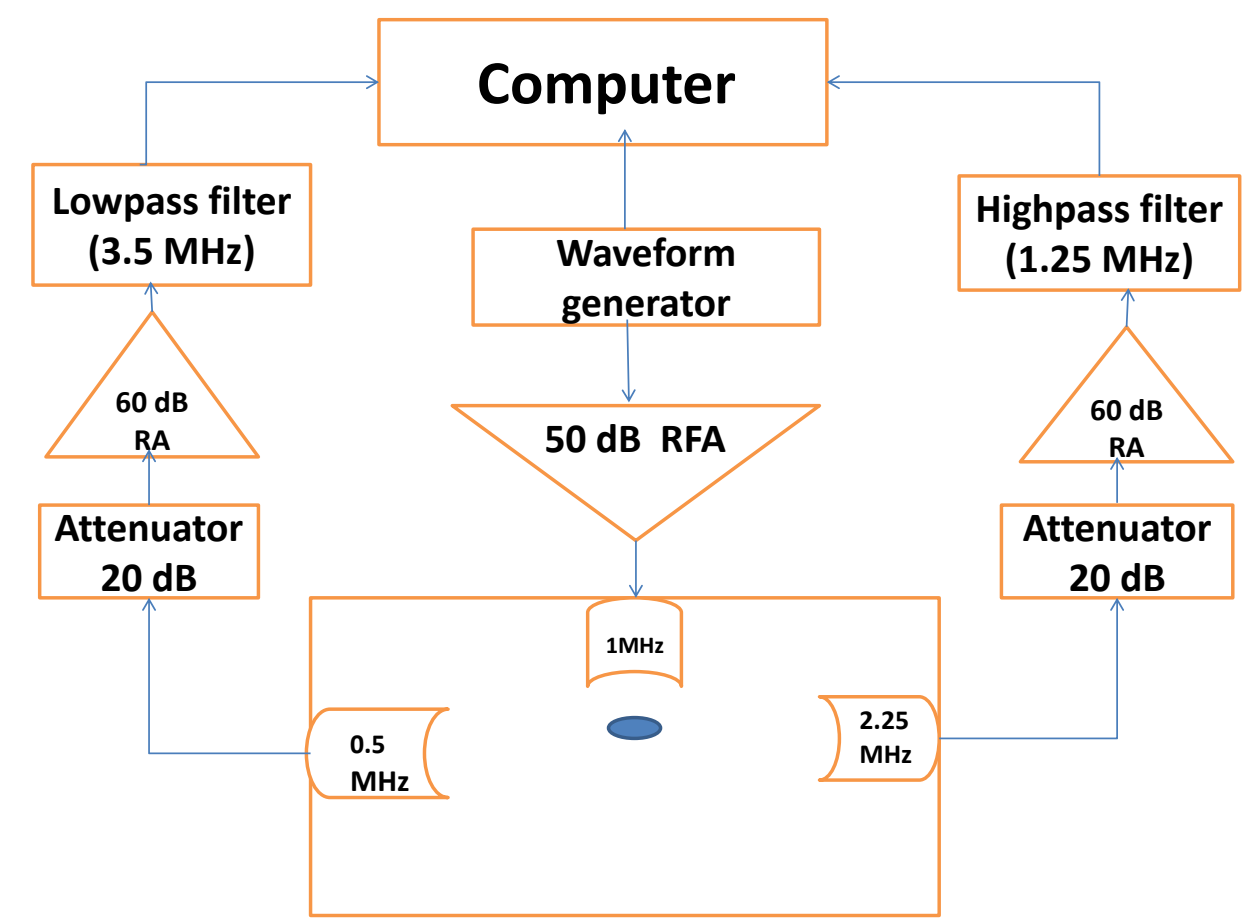

Figure 2.1: Schematic diagram of the PCD experimental setup. The bubbles emissions were received passively with 0.5 and $2.25 \mathrm{MHz}$ transducers with an idea of detecting subharmonic and harmonics. The pulses were transmitted with $1 \mathrm{MHz}$ transducer.

The pulses were transmitted to the RF power amplifier (240L, 50dB, ENI Inc. NY, Rochester, USA) that amplified the signals and drove a single-element spherically focused 1MHz transducer (IL0108HP, Valley Fisher Inc, Hopkinton, MA). PCD experiments were performed at room temperature in degassed water and with 3D micro-positioning systems graduated in mm scale for transducer positioning and 3D movable sample holder to ascribe its position. The sample was placed in a cylindrical tube at the focal volume which had dimensions of $10 \mathrm{~mm}$ in length and $8 \mathrm{~mm}$ in diameter. It was sealed with the mylair membrane and a magnetic stirrer was placed underneath for uniform mixing of the MBs suspension. The bubble emissions were collected passively by two PCD transducers at $2.25 \mathrm{MHz}$ and $0.5 \mathrm{MHz}$ (Valley Fisher Inc., Hopkinton, MA), that were positioned 
at 90 degrees to the treatment transducer at the focus as shown in the Figure 2.2. The PCD transducers were aligned perpendicular to the treatment transducer that enabled high spatial control over the region for which signals were collected because scattered signals only came from the MBs in the small overlapping confocal region. The acoustic emission signals collected from the MB suspension were amplified with a low noise (RF) amplifier (60dB, Miteq, USA) and passed through the attenuator (NAT-20, 20dB) and low pass filter $(3.5 \mathrm{MHz})$ or high pass filter $(1.25 \mathrm{MHz})$ to the computer.

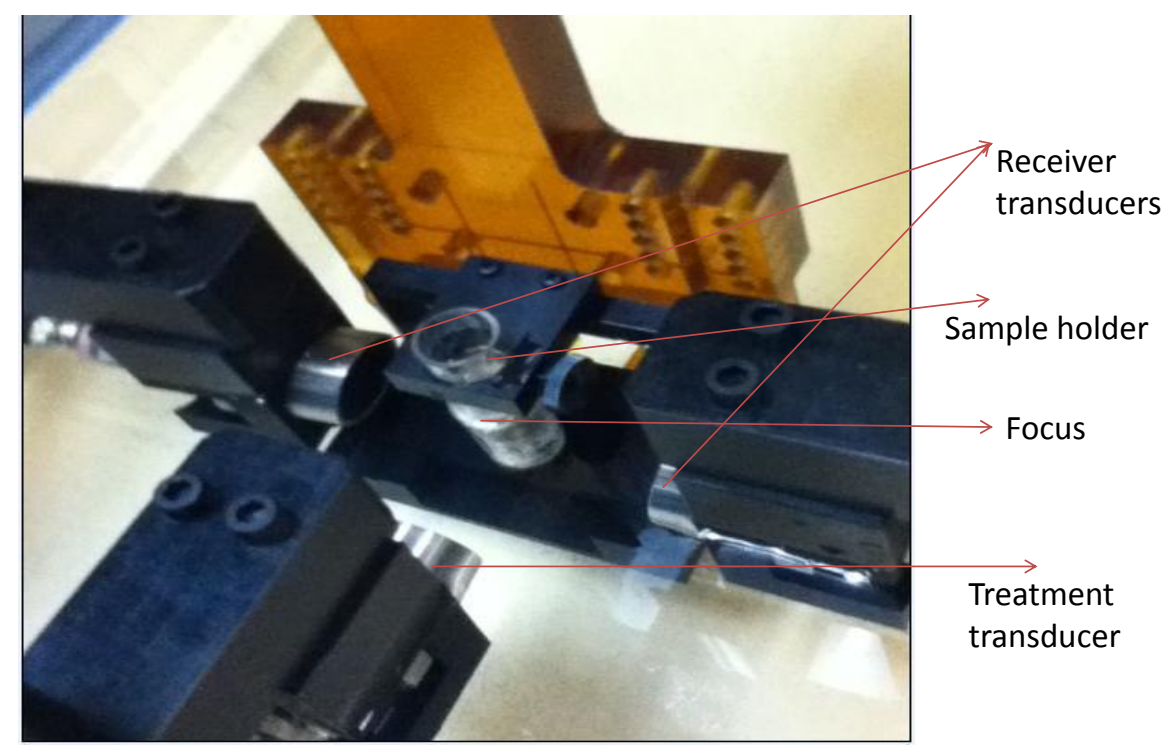

Figure 2.2: A photograph showing the transducer alignment at the focus. The two receiver transducers on left and right were perpendicular to the source transducer. The sample holder was sealed with mylair membrane. All the devices had 3D micropositioning system graduated in $\mathrm{mm}$ scale for their positioning. 


\subsubsection{Exposure protocol}

Before any experiments, a water tank of dimension $(50 \times 30 \times 20) \mathrm{cm}^{3}$ was half filled with degassed water. The calibration for the focus and positioning of transducers was performed using the 3D positioning calibrated system and with standard technique of cross needle measurement. The maximum value of the pulse-echo signal from needle, seen on the oscilloscope when the ultrasound is on, determines the centre of the focus. The focus was at a distance of $4.9 \mathrm{~cm}$, and $4.3 \mathrm{~cm}$ away from 2.25 and $0.5 \mathrm{MHz}$ receiver transducers and $4.5 \mathrm{~cm}$ away from $1 \mathrm{MHz}$ treatment transducer. The magnetic stirrer was placed underneath sample holder for the uniform mixing of the MB suspension. Water sample $(1 \mathrm{ml})$ in the absence of MBs was placed with syringe, inside the sample holder tube which was constructed with mylair membrane sealed around it at the focus. The cylindrical shaped focal volume has a dimension of $10 \times 8 \mathrm{~mm}$. The power amplifier was turned on and computer program was initiated to record the acquired signals in acquisition card. The exposure was started by triggering the function generator with sequenced pulse containing PRP of $1 \mathrm{~ms}$, repeated 1000 times for the total exposure time of $1 \mathrm{~s}$ (Figure 2.3). Experiments were conducted varying acoustic pressure (PNP) of $0,0.5,1.1,1.5 \mathrm{MPa}$ and pulse duration of $5 \mu \mathrm{s}$ and $30 \mu \mathrm{s}$. The transmit pressure amplitude was varied by controlling the function generator output voltage $(0-600 \mathrm{mV})$ through Arb connection software (Tabor Electronic Ltd, NY, USA). After water signals (reference acquisition data) recording were completed the MB samples were prepared at $1 \%$ concentration and were placed in the focal volume and the signals were recorded for the given exposure conditions. For each of the exposure conditions the sample was replaced with new MBs sample and the signal received with transducers were acquired in data acquisition system. 


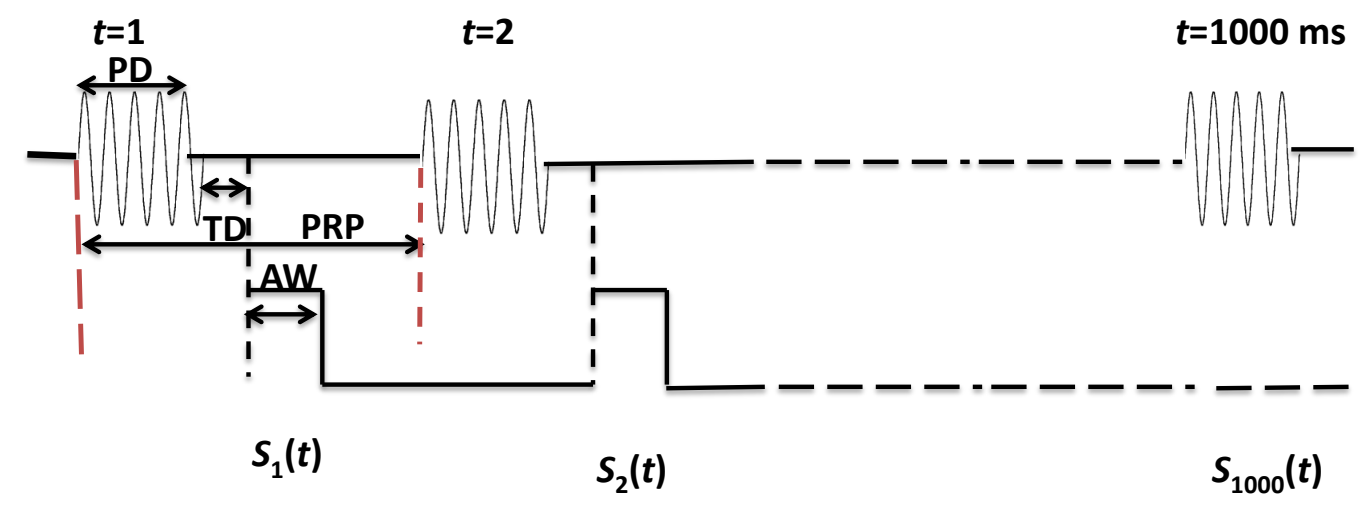

Figure 2.3: Diagram showing the exposure pulse sequence and data acquisition timing. PD : pulse duration (5 and $30 \mu \mathrm{s}), \mathrm{PRP}:$ pulse repetition period $(1 \mathrm{~ms})$, TD : trigger delay $(60 \mu \mathrm{s})$ and $\mathrm{AW}:$ acquisition window $(48 \mu \mathrm{s})$

\subsubsection{Data Acquisition}

The acoustic signals emitted by MBs and water were recorded in $1 \mathrm{~ms}$ time intervals, after the sequenced pulse was initiated (Figure 2.3). A total of 1000 signals (each having 6000 samples) were acquired at a sampling rate of $125 \mathrm{MHz}$ in an Alazar DSO acquisition system (ATS460-128M-S600726) with an acquisition window of length $48 \mu$ s for given exposure time of 1 second. Each acquisition, $s_{i}(t)$ where $\mathrm{i}=1$ to 1000 represents the acquisition events for each time points. The time delay of $60 \mu$ s was applied with respect to the trigger signal, which was necessary to account for the travel time from the source to the receiver transducers. The acquisition was stopped after acquiring the data for $1 \mathrm{~s}$ and the data was saved as MATLAB file for further analysis. 


\subsubsection{Transducer Specifications}

Three transducers were used in this project. A spherically focused single element $1 \mathrm{MHz}$ transducer (IL0108HP, Valpey Fisher Inc, Hopkinton, MA) with a diameter of $25 \mathrm{~mm}$, focal length of $4.5 \mathrm{~cm}$ and bandwidth of $\pm 6 \mathrm{~dB}$ was used for transmitting ultrasound pulse to the MB suspension while a $0.5 \mathrm{MHz}$ (IL0508HP, Valpey Fisher Inc, Hopkinton, MA) and a $2.25 \mathrm{MHz}$ (IL0208HP, Valpey Fisher Inc, Hopkinton, MA) transducers were used for measuring the acoustic signals from MBs. Both have a diameter of $25 \mathrm{~mm}$, focal lengths of 4.3 and $4.9 \mathrm{~cm}$ respectively and the bandwidth of $\pm 6 \mathrm{~dB}$.

\subsubsection{Transducer calibration}

All transducers were characterised using a calibrated needle hydrophone (Precision Acoustics Ltd, Dorchester, and Dorset, UK). In absence of an exposure chamber the pressure was measured at the focus of the transducer beam. The pressure field was characterized based on the graph shown below for $1 \mathrm{MHz}$ transducer (Figure.2.4). The peak negative pressure was used to characterize the acoustic amplitude.

\subsection{Microbubble agent}

Two MB agents (Definity and Artenga) were used in this study. Definity, Perflutren lipid microspheres, (Lantheus Medical Imaging, Billerica, MA, USA) are FDA approved ultrasound imaging contrast agents composed of the octofluoropropane (C3F8) gas core stabilised by phospolipid shells. The container vials have a concentration of $1.2 \times 10^{10}$ bubbles $/ \mathrm{mL}$, and average diameters of $1.1-3.3 \mu \mathrm{m}$, with $98 \%$ less than $10 \mu \mathrm{m}$ according to the manufacturer. The Definity vial was activated by shaking it with Vial-Mix (Lan- 


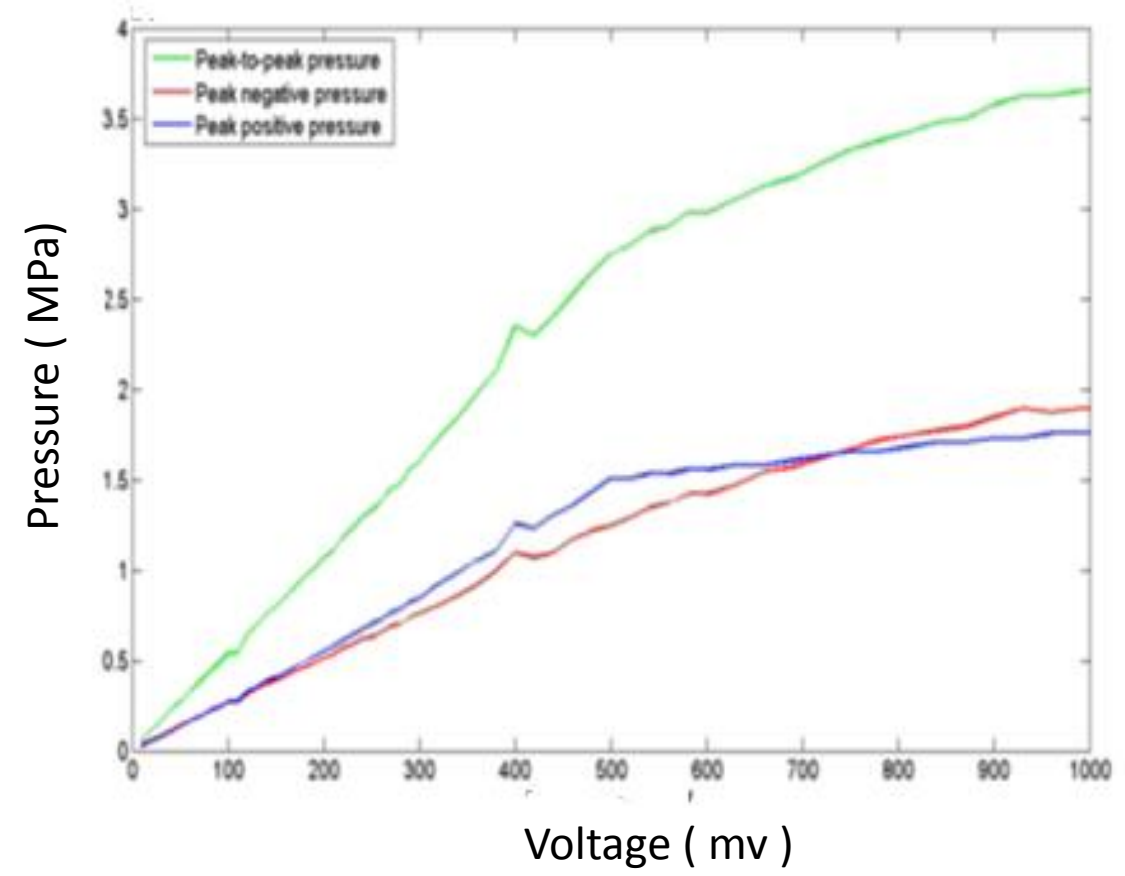

Figure 2.4: $1 \mathrm{MHz}$ transducer calibration curve. Y- axis representing pressure in MPa. The $\mathrm{X}$-axis represents voltage in $\mathrm{mV}$. The Green curve is peak-to-peak pressure, the red curve absolute value of peak negative pressure and the blue curve peak positive pressure.

theus Medical Imaging, Billerica, MA, USA) for 45 seconds and was equilibrated for 5 minutes at room temperature. The vial was agitated for 10 seconds by hand, inverted for 30 seconds and was vented with an 18-gauge needle. Finally MBs were transferred to a syringe and were diluted in water at the desired concentration. Artenga MB, an ultrasound contrast agent, currently in clinical trials contains perflurocarbon gas enclosed in a lipid shell. It has a mean bubble diameter of 2 microns and a concentration of $1 \times 10^{9}$ microbubbles/mL (Artenga Inc, Ottawa, canada). The MB suspension was prepared according to the manufacturer instructions (Artenga Inc, 2008).

A Coulter Counter System (Multisizer IV, Beckman Coulter Inc, Fullerton, CA) was used for the MB size distribution measurements. The size distribution of MBs in a $5 \mu \mathrm{L}$ 
volume were measured using a $20 \mu \mathrm{m}$ aperture tube. Measurements were performed for Artenga MB having volume weighted diameter of 1-20 $\mu \mathrm{m}$ in range. Figure 2.5 shows the Coulter Counter results where the average MBs size distribution was approximately $2-4 \mu \mathrm{m}$.

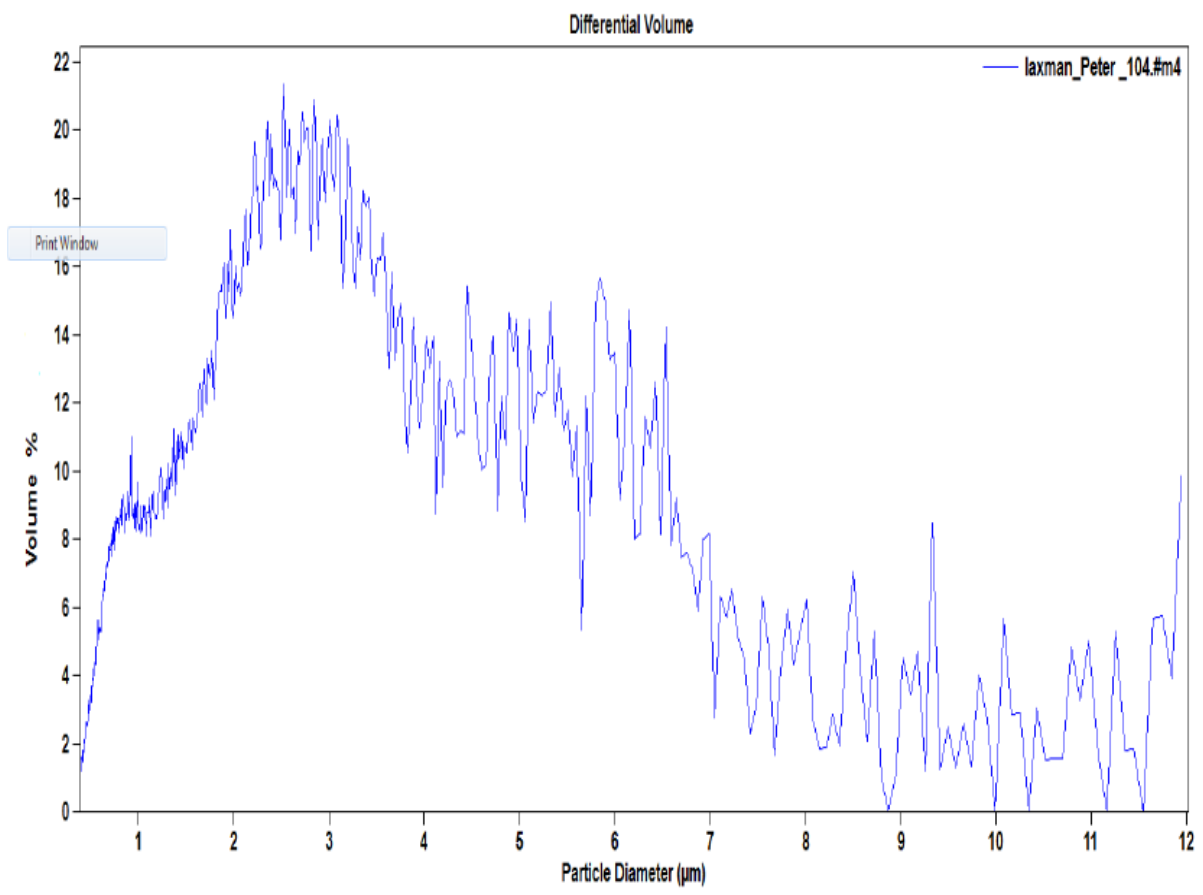

Figure 2.5: Volume weighted size distribution measurements with Coulter Counter for Artenga MBs .

\section{$2.3 \quad$ Experiments performed}

PCD experiments were performed with two MBs (Artenga and Definity) at various acoustic pressure, pulse duration and microbubble sizes. The parameters used in the experiments are discussed in previous subsection (2.1.1). The list of PCD experiments performed during study period of 2 years with some specifications are shown in Table 2.1 
Table 2.1: List of PCD experiments: where $\mathrm{PD}=$ pulse duration, $\mathrm{PNP}=$ Peak negative pressure and FIL/UNF represents filtered/unfiltered bubbles and NOE represents numbers of experiments.

\begin{tabular}{|l|c|c|c|c|c|c|}
\hline Exp.No & MB type & PD(us) & PNP(MPa) & Receiver(MHz) & FIL/UNF & NOE \\
\hline 1 & ART & 30 & $0-1.5$ & 2.25 and 0.5 & UNF & 3 \\
\hline 2 & DEF & 30 & $0-1.5$ & 2.25 and 0.5 & UNF & 3 \\
\hline 3 & ART & 5 & $0-1.5$ & 2.25 and 0.5 & UNF & 3 \\
\hline 4 & DEF & 5 & $0-1.5$ & 2.25 and 0.5 & UNF & 3 \\
\hline 5 & DEF & 30 & $0-1.5$ & 2.25 and 0.5 & FIL & 1 \\
\hline
\end{tabular}

\subsection{Data Analysis}

\subsubsection{Microbubble behaviour}

The acquired acoustic signals $\left(s_{i}(\mathrm{t})\right.$ from MBs at $1 \mathrm{~ms}$ time interval were analyzed using MATLAB (Mathworks, Natick, MA). The post triggered samples (24-48us) were analyzed using the rectangular window for the frequency response. The frequency response were obtained with FFT algorithm in MATLAB using the transformation equation $\left[S_{i}(f)\right]=$ $20 \log _{10} \operatorname{FFT}\left[s_{i}(t)\right]$. At $10 \mathrm{~ms}$ after exposure the equation used was

$$
\left[S_{10}(f)\right]=20 \log _{10} \operatorname{FFT}\left[\left(\mathrm{s}_{10}(t)\right]\right.
$$

Moreover the MB acoustic response was analysed by plotting spectrograms which showed the time dynamics of MB emissions and their activities in term of frequency content over insonation time. Furthermore cavitation MB activities were measured in terms of cavitation dose. 


\subsubsection{Cavitation dose}

The response of MBs varied with insonation time. The cavitation dose of MB response was calculated based on the RMS FFT Algorithm as described by (39). The mathematical expression for calculating cavitation dose in this project is given as

$$
C D_{R M S}=\int_{f-\triangle f}^{f+\triangle f}\left|S_{i}(f)\right|^{2} d f
$$

where $\mathrm{i}=1-1000$ represents the acquisition events for each time points, $S_{i}(f)$ represents the FFT of $s_{i}(t) . \triangle f=0.2 \mathrm{MHz}$ and $\mathrm{f}=(0.5,1.5,2 \mathrm{MHz}$ for the subharmonic, ultraharmonic and second harmonic frequency spectrum respectively). The interval between $\mathrm{f}-\triangle f$ and $\mathrm{f}+\triangle f$ represents the frequency window for calculating the cavitation dose.

In this project the RMS FFT amplitude vs time plot (cavitation dose) was calculated for 1000 signals each acquired at $1 \mathrm{~ms}$ time interval at desired frequency windows of sub-

harmonic $(0.5 \pm 0.2 \mathrm{MHz})$ second harmonic $(2 \pm 0.2 \mathrm{MHz})$ and ultraharmonic $(1.5 \pm 0.2 \mathrm{MHz})$ frequencies. In addition the integrated cavitation dose (ICD) at given frequency windows over the exposure time of $1 \mathrm{~s}$ were obtained following an idea as discussed by (56; 39). The ICD for each spectrum (subharmonic, harmonic, and ultra-harmonics response) was defined as the area under the RMS FFT amplitude curve over the exposure time of $1 \mathrm{~s}$. The following mathematical expression was used to calculate the integrated cavitation dose

$$
I C D=\sum_{i=1}^{1000}\left|C D_{R M S}\right| \triangle t
$$

where $\triangle t$ represents the time difference between two acquisition events which is $1 \mathrm{~ms}$. A schematic diagram representing data analysis algorithm is shown in Figure 2.6 
a

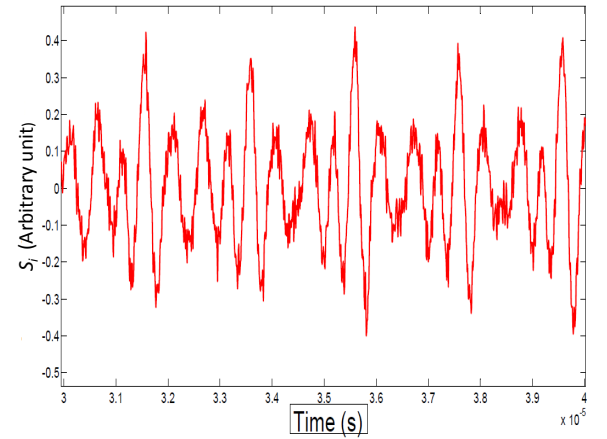

C

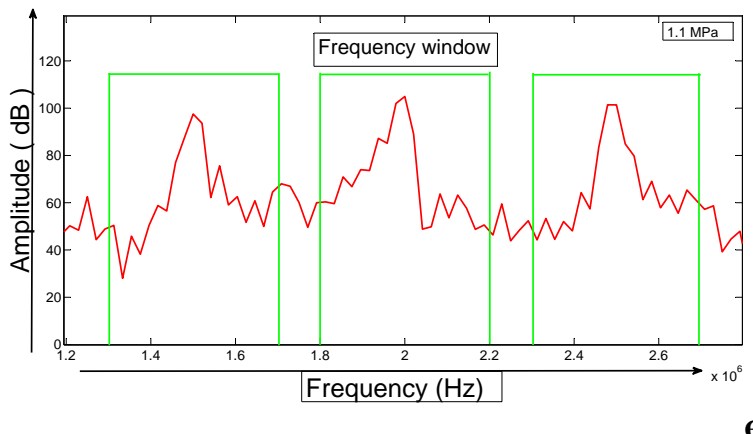

b

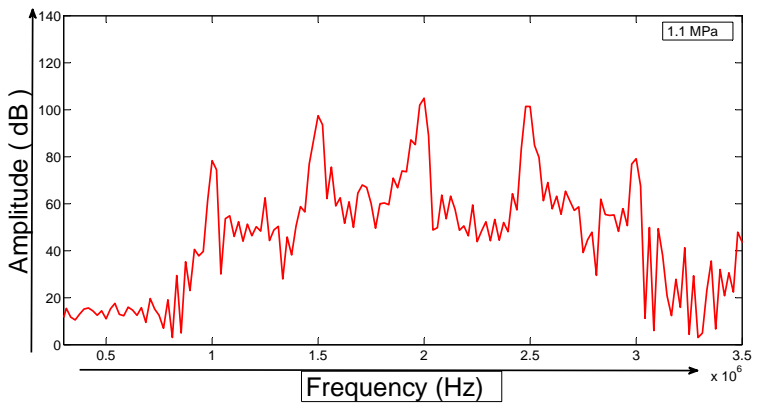

d

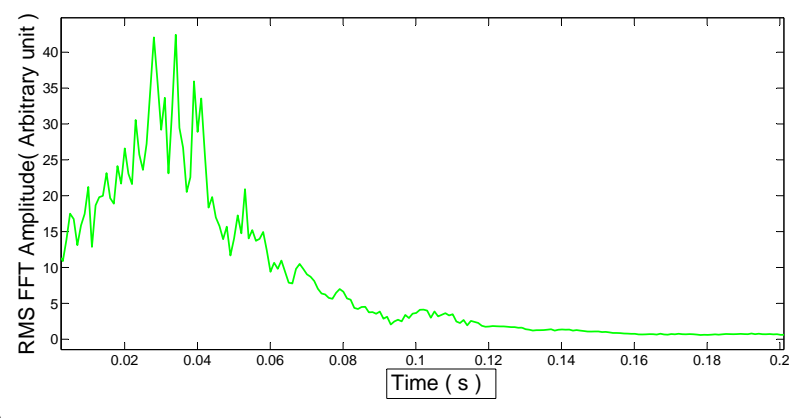

e

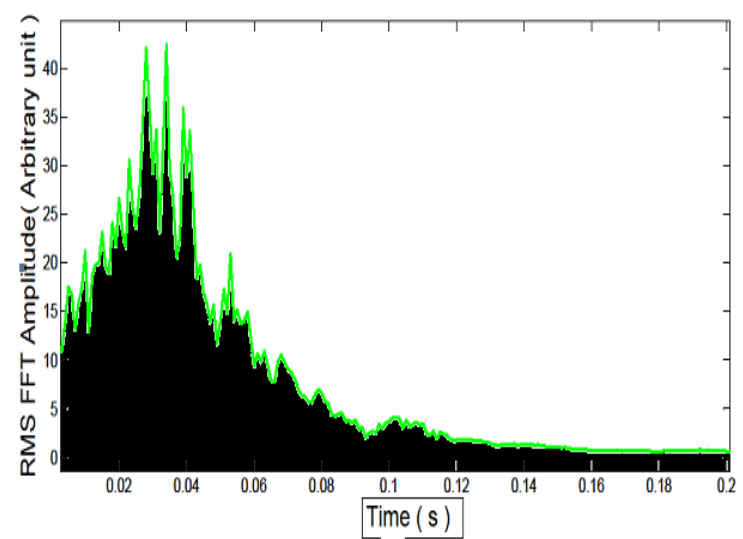

Figure 2.6: Data analysis algorithm: (a) Time domain signal at $10 \mathrm{~ms}\left[s_{10}(t)\right]$. (b) represents frequency response of TD signal obtained using equation 2.1. (c) showing the frequency window at ultraharmonics and second harmonic frequency spectra for calculating the cavitation dose. (d) graph representing cavitation dose obtained using equation 2.1. (e) ICD was obtained integrating the cavitation dose from the shaded region over the exposure time of $1 \mathrm{~s}$ using equation 2.2 . 


\section{Chapter 3}

\section{Results}

In this chapter, the acoustic characteristics of the two MBs (DEF and ART) exposed at varying acoustic pressures (0-1.5 MPa) and pulse duration (5 and 30 cycles) for insonation time of 1s was investigated. The MB acoustic response are shown in Section 3.1 and the cavitation dose results in Section 3.2.

\subsection{MB acoustic behaviour}

\subsubsection{Effect of acoustic pressure (PNP)}

The effect of acoustic pressure (PNP) on acoustic MB behaviours are shown in Figures 3.1 to 3.4 which presents the PCD results for different PNP. MBs exhibit nonlinear and chaotic behaviours (Figure 3.1 and 3.2). Spectrograms associated with the acoustic response of the MB are presented in Figure 3.3 and FFT spectra at various time points are shown in Figure 3.4.

The acoustic behaviour of ART MB exposed to 30 cycle pulses at PNP (0-1.5 MPa) depends on the applied PNP (Figure 3.1 and 3.2). The time domain signals received 

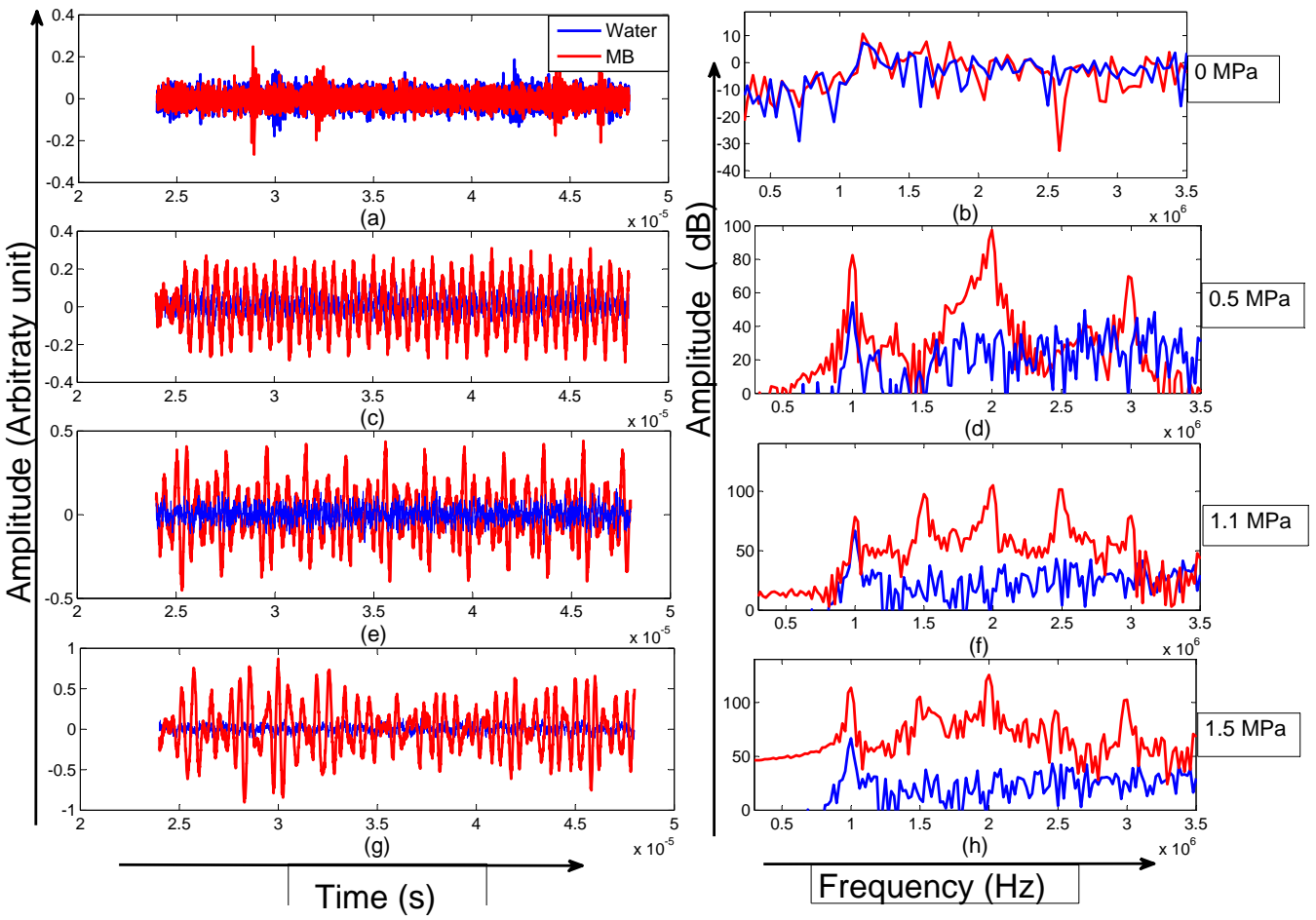

Figure 3.1: ART MB behaviours (red) and water (blue) as a function of PNP at $10 \mathrm{~ms}$ after exposure. Figures $(\mathrm{a}, \mathrm{c}, \mathrm{e}, \mathrm{g})$ and $(\mathrm{b}, \mathrm{d}, \mathrm{f}, \mathrm{h})$ represent the time domain signals and their corresponding FFT response at $(0,0.5,1.1,1.5) \mathrm{MPa}$ respectively. The vertical axis and horizontal axis for TD signal represents amplitude (arbitrary units) and time (s) while it is pressure amplitude $(\mathrm{dB})$ and frequency $(\mathrm{Hz})$, for FFT figures. MBs and water were insonated with 30 cycle $1 \mathrm{MHz}$ pulse at $\mathrm{PRF}$ of $1 \mathrm{KHz}$ and were received with $2.25 \mathrm{MHz}$ transducers with highpass filter of $1.25 \mathrm{MHz}$.

with the $2.25 \mathrm{MHz}$ transducer and their corresponding FFT spectra are shown in Figure 3.1 at10 ms after MB were exposured to ultrasound. At $0 \mathrm{MPa}$, the MB exhibits no sign of oscillation and its corresponding FFT spectrum is featureless Figure 3.1(a,b). At 0.5 $\mathrm{MPa}$, the MB shows weak non-linear oscillation with an emission of the fundamental $(f)$, second harmonic $(2 f)$ and third harmonic $(3 f)$ Figure 3.1(c, d). The pressure amplitude are higher than water by $67 \mathrm{~dB}$ for $(2 f)$ and $45 \mathrm{~dB}$ for $(3 f)$. At $1.1 \mathrm{MPa}$, the MBs undergo strong nonlinear oscillation with an emission of second harmonic $(2 f)$, ultra- 
harmonics $(1.5 f, 2.5 f)$ and third harmonic $(3 f)$ greater than water by $73,76,91$, and 52 dB respectively Figure $3.1(\mathrm{e}, \mathrm{f})$. At 1.5 MPa, the MBs exhibit chaotic behavior with an emission of broadband spectra containing second harmonic $(2 f)$, ultra-harmonics (1.5f, $2.5 f$ ) and third harmonics $(3 f)$ by $98,83,66$ and $67 \mathrm{~dB}$ greater than water respectively (Figure $3.1(\mathrm{~g}, \mathrm{~h})$ ). More detail analysis of the time domain signal is discussed in the appendix (Figure A.1).
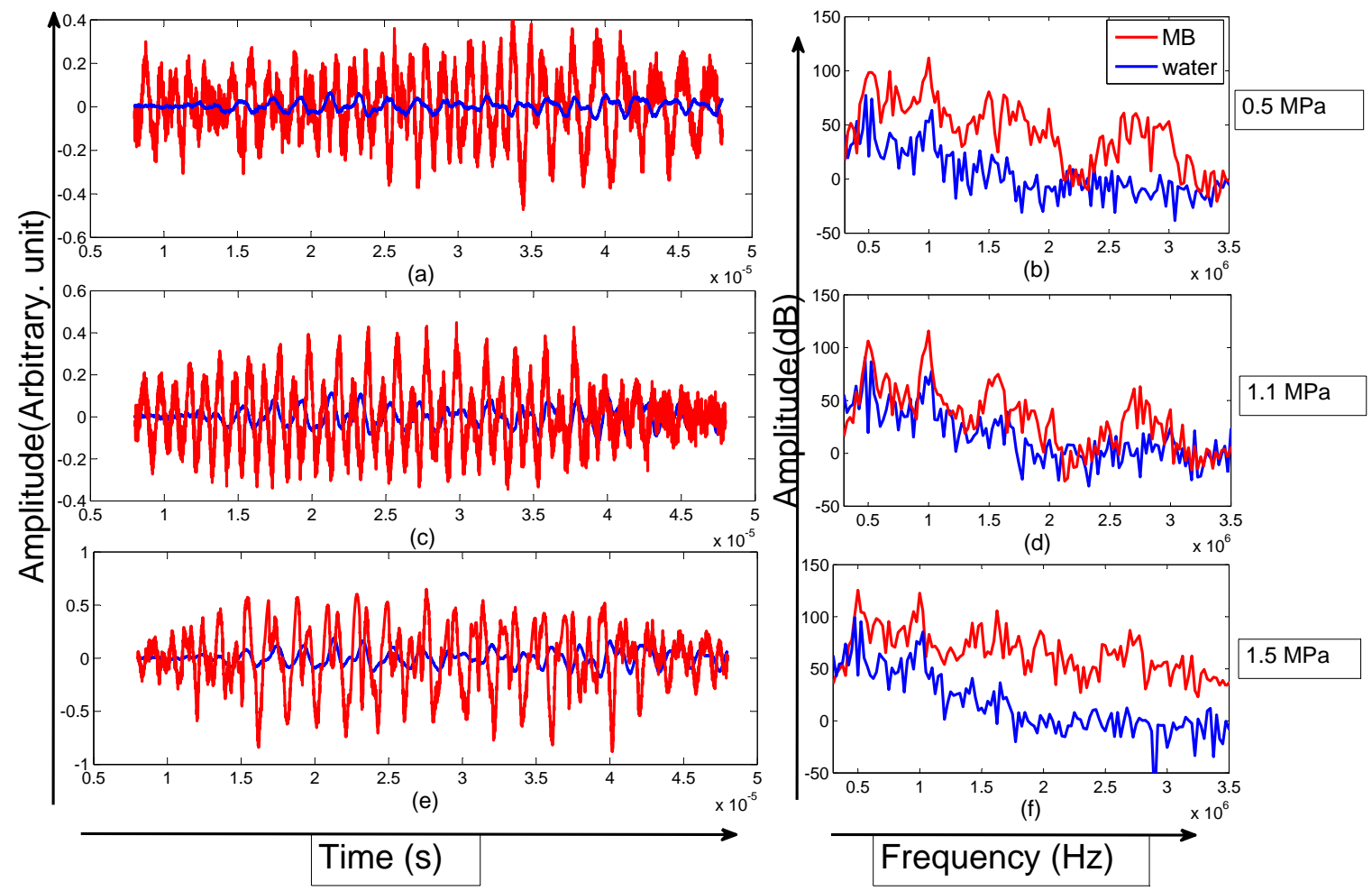

Figure 3.2: ART MB behaviours (red) and water (blue) as a function of PNP at $10 \mathrm{~ms}$ after their exposure. Figures $(\mathrm{a}, \mathrm{c}, \mathrm{e})$ and $(\mathrm{b}, \mathrm{d}, \mathrm{f})$ represent the time domain signals and their corresponding FFT spectra at $(0.5,1.1,1.5) \mathrm{MPa}$ respectively. The vertical axis and horizontal axis for TD signal represents amplitude (arbitrary unit) and time (s) while it is pressure amplitude $(\mathrm{dB})$ and frequency $(\mathrm{Hz})$, for FFT figures. MBs and water were insonated with 30 cycle $1 \mathrm{MHz}$ pulse at $\mathrm{PRF}$ of $1 \mathrm{KHz}$ and were received with $0.5 \mathrm{MHz}$ transducers with lowpass filter of $3.5 \mathrm{MHz}$.

The time domain signals received with the $0.5 \mathrm{MHz}$ transducer for $10 \mathrm{~ms}$ insonation 
time after exposure of MB to ultrasound and their corresponding FFT spectra are shown in Figure 3.2. At 0.5 MPa, the MBs exhibit weak non-linear oscillation (unequal and irregular expansion and contraction) with an emission of the subharmonic $(f / 2)$ of magnitudes $21 \mathrm{~dB}$ higher than water Figure 3.2 (a, b). At $1.1 \mathrm{MPa}$, the MBs undergo asymmetric oscillations which exhibit expansion-like behaviour and emit the subharmonic frequency spectrum of magnitude $18 \mathrm{~dB}$ greater than water Figure $3.2(\mathrm{c}, \mathrm{d})$. At $1.5 \mathrm{MPa}$, the MB exhibits compression-like behavior and emit the subharmonic frequency spectrum of magnitude $30 \mathrm{~dB}$ higher than water Figure $3.2(\mathrm{e}, \mathrm{f})$. More detail analysis of the time domain signal is discussed in the appendix (Figure A.2).

The time dynamics of ART MB acoustic behaviour, received with $0.5 \mathrm{MHz}$ and 2.25 $\mathrm{MHz}$ transducers, are presented in spectrograms Figure 3.3. It is seen that with an increase of pressure, nonlinear behaviour of the MBs increases. The time dynamic results indicate that MBs undergo large scale oscillations with emission of the higher amplitude frequency spectra up to $200 \mathrm{~ms}$ following the lower amplitude signals with longer insonation times Fig 3.3 (b, d, f). At $1.1 \mathrm{MPa}$, the frequency spectra from the MBs nonlinear oscillation with respect to insonation time are observed to be very strong during the first $200 \mathrm{~ms}$. At $1.5 \mathrm{MPa}$, the MBs exhibit spectra of higher magnitude for the first $100 \mathrm{~ms}$. Following this, the signals disappear for $200 \mathrm{~ms}$ and reappear at 300 to $500 \mathrm{~ms}$ Figure 3.3 (f) as shown by the arrow. The spectrograms for water at $1.1 \mathrm{MPa}$ Figure $3.3(\mathrm{~h})$ show no other frequency spectrum except the fundamental frequency $(f)$. Similar results from experiment 2 and experiment 3 are shown in appendix (Figure 5.8). Spectrograms with the $0.5 \mathrm{MHz}$ transducer have shown that the MBs frequency spectra for subharmonic have greater amplitude as the pressure increases. These results are from different experiments than that received with $2.25 \mathrm{MHz}$ transducers.

The frequency spectra from ART MB, detected with the $2.25 \mathrm{MHz}$ transducer at 


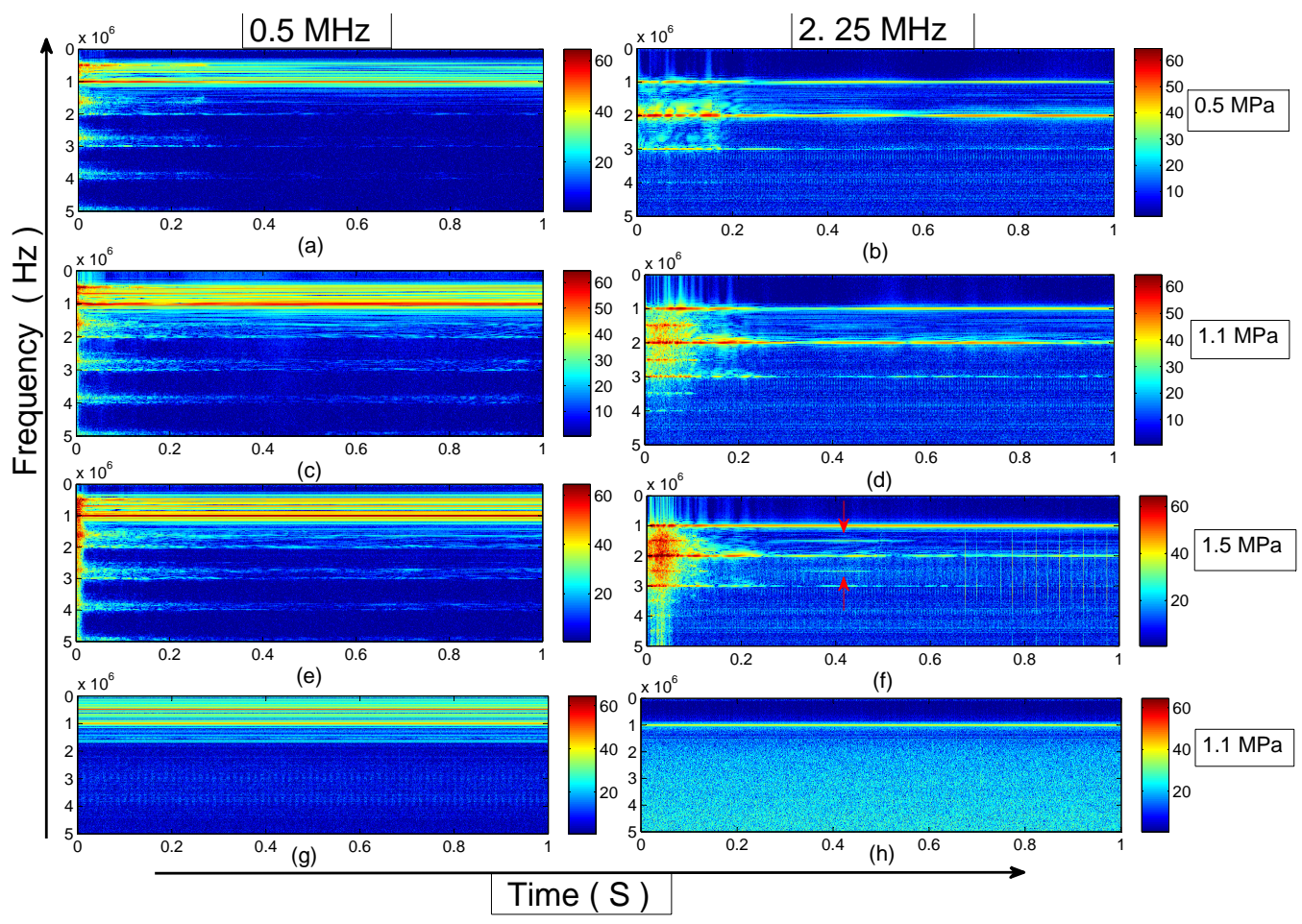

Figure 3.3: ART MB spectrograms as a function of PNP for signals received with 0.5 and $2.25 \mathrm{MHz}$ receiver transducers. Results are shown for $(0.5,1.1,1.5) \mathrm{MPa}$ for MB Figure 3.3 (a to f), and water at $1.1 \mathrm{MPa}(\mathrm{g}, \mathrm{h})$. The vertical axis on the spectrogram represents frequency $(\mathrm{Hz})$ and horizontal axis represents time $(\mathrm{s})$. The color bar represents the frequency spectral amplitude, with red the highest and blue the lowest.

different time points (Figure 3.4) demonstrate that the FFT peak pressure amplitude increases with the increase of pressure while it decreases with insonation time. The time points were chosen based on the result of the spectrogram Figure 3.3. At 0.5 MPa, the peak pressure amplitude of the second $(2 f)$ and third harmonic $(3 f)$ decreases from 101 to $55 \mathrm{~dB}$, and 70 to $43 \mathrm{~dB}$ respectively, within $240 \mathrm{~ms}$ insonation time. At $1.1 \mathrm{MPa}$, it decreased from 103 to 15, 110 to 74,101 to 13 and 98 to $38 \mathrm{~dB}$ within an insonation time of $300 \mathrm{~ms}$, for the ultraharmonic $(1.5 f)$, second harmonic $(2 f)$, ultraharmonic $(2.5 f)$ and third harmonic $(3 f)$ frequencies respectively. At 1.5 MPa, the peak pressure amplitude of 

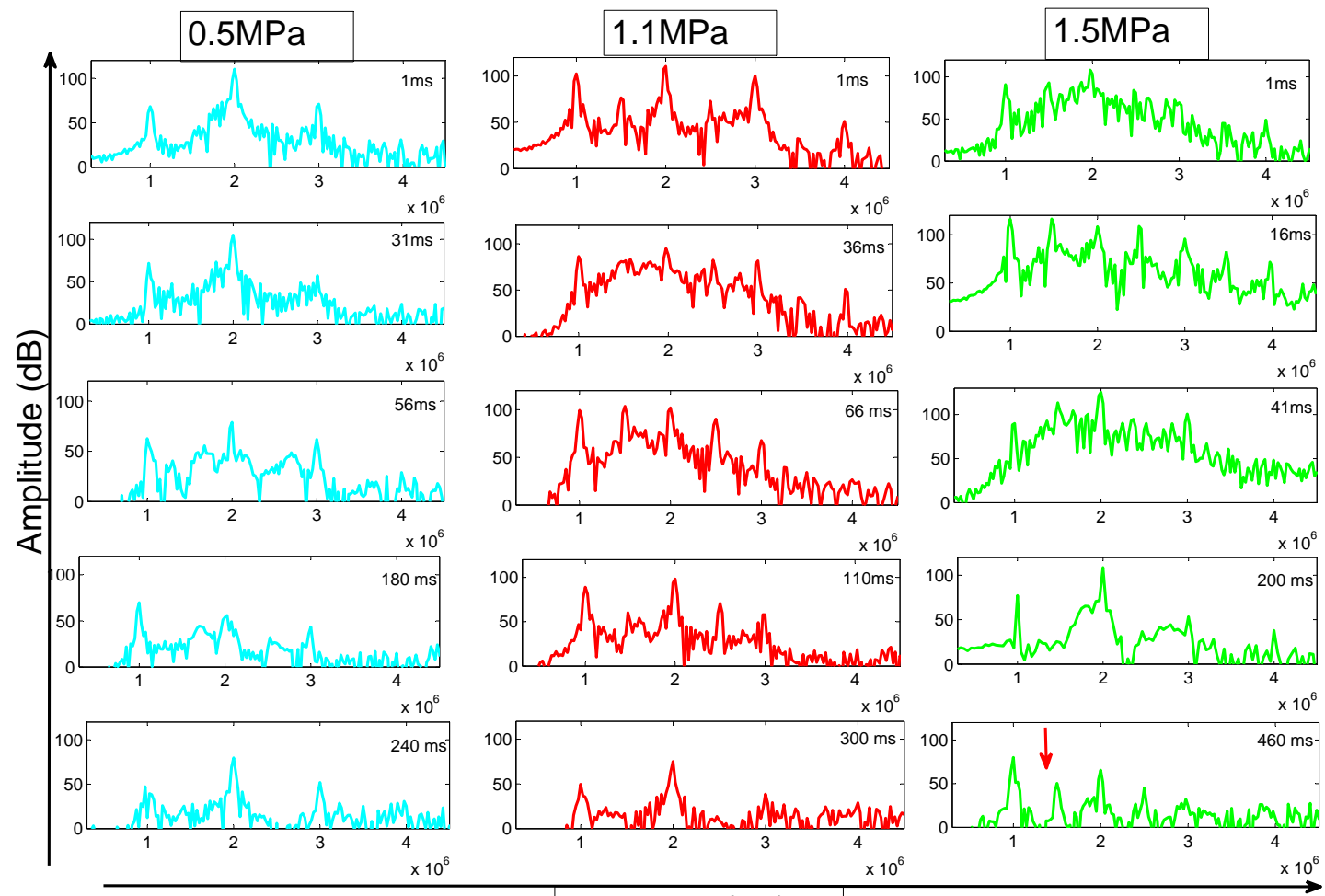

Frequency $(\mathrm{Hz})$

Figure 3.4: FFT spectra of ART MB at various time points for the data received with $2.25 \mathrm{MHz}$ transducers. The signals in blue colour represents FFT spectra at various time points for $0.5 \mathrm{MPa}$, red for 1.1 $\mathrm{MPa}$ and green for 1.5 MPa.The vertical axis represents pressure amplitude $(\mathrm{dB})$ and horizontal axis represents frequency $(\mathrm{Hz})$.

the ultraharmonic $(1.5 f)$, second harmonic $(2 f)$, ultraharmonic $(2.5 f)$ and third harmonic (3f) decreased from 116 to 50,127 to 65,108 to 45, 95 to $32 \mathrm{~dB}$ respectively within 500 ms insonation time. 


\subsubsection{Effect of acoustic pressure and pulse duration on MB type}

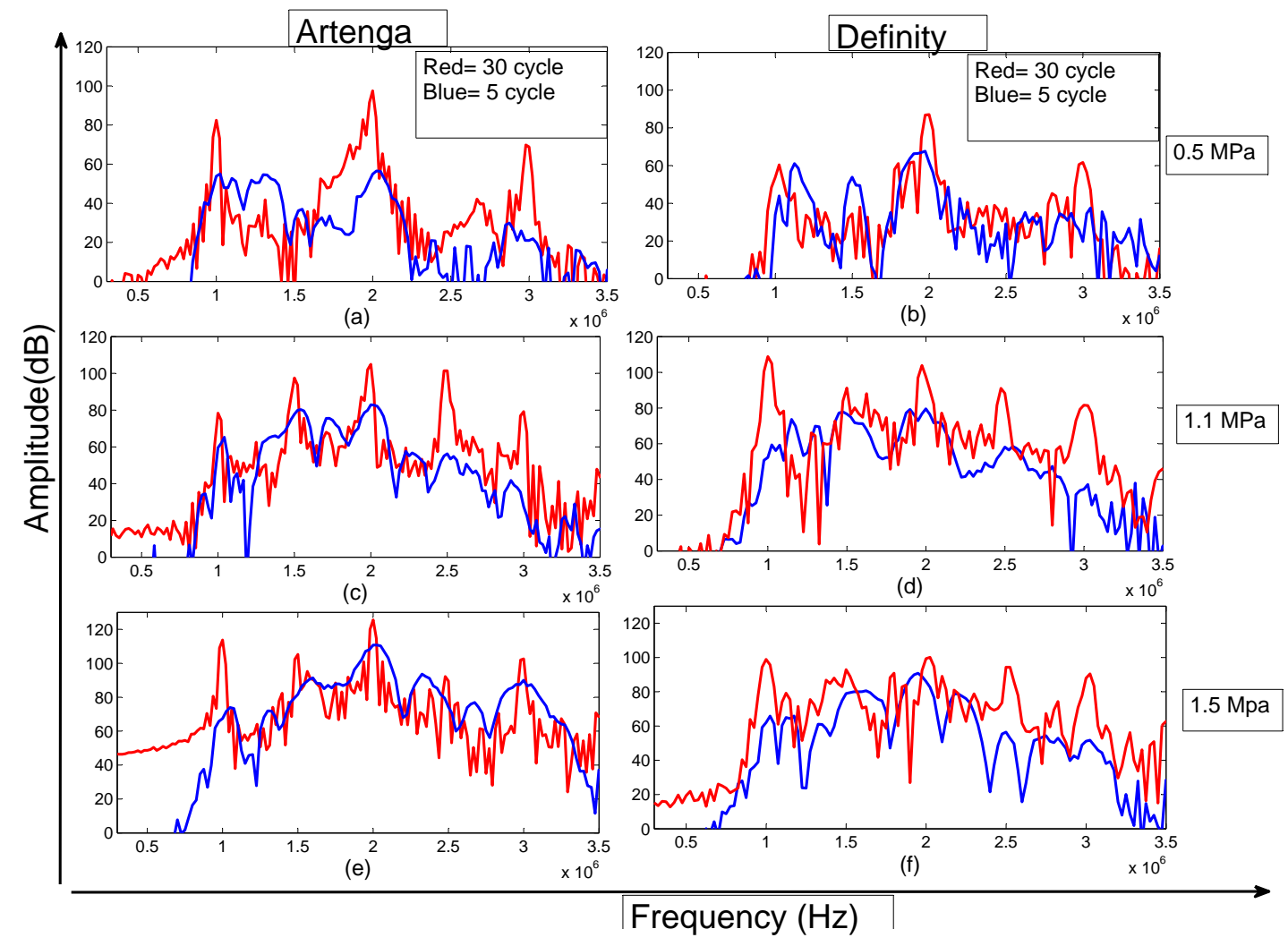

Figure 3.5: FFT spectra for ART MB at $10 \mathrm{~ms}$ insoonation time and DEF MB at $43 \mathrm{~ms}$ insonation time (for 30 cycles) and $210 \mathrm{~ms}$ insonation time (for 5 cycles) after exposure and $\mathrm{PNP}$ of $0.5,1.1,1.5 \mathrm{MPa}$. Blue color represent 5 cycles and red colour represents 30 cycles pulse duration, and the signals were received with $2.25 \mathrm{MHz}$ transducer. The vertical axis represents pressure amplitude $(\mathrm{dB})$ and the horizontal axis represents frequency $(\mathrm{Hz})$.

The frequency spectra of both MBs (Artenga and Definity) exposed to 30 cycle and 5 cycle pulse duration at acoustic pressure of $0.5,1.11 .5 \mathrm{MPa}$ are shown in Figure 3.5. ART MB FFT spectra for both pulse duration are shown at $10 \mathrm{~ms}$ insonation time after exposure while DEF MB FFT spectra are shown at $43 \mathrm{~ms}$ for 30 cycle pulse duration and $210 \mathrm{~ms}$ for 5 cycle pulse duration after exposure. These time points for DEF MB were chosen based on the results shown in Figure 3.7 where the MB activities were significant 
to analyse and $10 \mathrm{~ms}$ was choosen based on Figure 3.3 and 3.6. Results demonstrate that the FFT spectrum of 5 cycle exposure were different from 30 cycle exposure. Similar results from second set of experiment are shown in appendix (Figure B.6).

The ART and DEF MBs, both at 5 cycles pulse duration have broader FFT spectra with lower pressure amplitudes compared to 30 cycles frequency responses Figure 3.5. Large difference in the pressure amplitude can be seen with a range of 8 to $47 \mathrm{~dB}$ among the harmonics and ultraharmonics. At 5 cycle pulse duration ultraharmonic signal (1.5 $\mathrm{MHz}$ ) of lower amplitude was seen at $0.5 \mathrm{MPa}$ (Figures 3.5 and B.6). This can be clearly seen from the spectrograms results (Figures 3.7, B.3 and B.4) where lower amplitude frequency spectrum is visible at $1.5 \mathrm{MHz}$. For 30 cycle pulse duration results, this signal was neither seen in FFT spectra (Figures 3.5 and B.6) nor in spectrograms (Figures 3.3, B.1 and B.2).

In addition ART and DEF MBs at 5 and 30 cycles pulse duration have different frequency response (Figure 3.5). The frequency spectra for Definity MB (5 cycle) have the higher peak pressure amplitude of ultraharmonic $(1.5 f)$, second harmonic $(2 f)$, ultraharmonic $(2.5 f)$ and third harmonic $(3 f)$ than the corresponding Artenga MBs spectra at 0.5 and $1.1 \mathrm{MPa}$. The difference in the peak pressure amplitude was 17, 11, aand 16 $\mathrm{dB}$ at $0.5 \mathrm{MPa}$ (no 2.5f) and 3, 4, 5 , and $1 \mathrm{~dB}$ at $1.1 \mathrm{MPa}$. However at $1.5 \mathrm{MPa}$ it was opposite and the difference in peak pressure amplitude were 3, 20, 18, and $39 \mathrm{~dB}$ higher for ART MBs.

For 30 cycle pulse duration, ART MBs have higher peak pressure amplitudes than Definity for most of the FFT spectra at all pressures (Figure 3.5). The peak pressure amplitude differences were 11 and $8 \mathrm{~dB}$ for second $(2 f)$ and third harmonics $(3 f)$ at 0.5 MPa. At 1.1 MPa the differences were 7, 1, 11, and $2 \mathrm{~dB}$ for the ultraharmonic (1.5-emphf), second harmonic $(2 f)$, ultraharmonic $(2.5 f)$ and third harmonic $(3 f)$ respectively, 


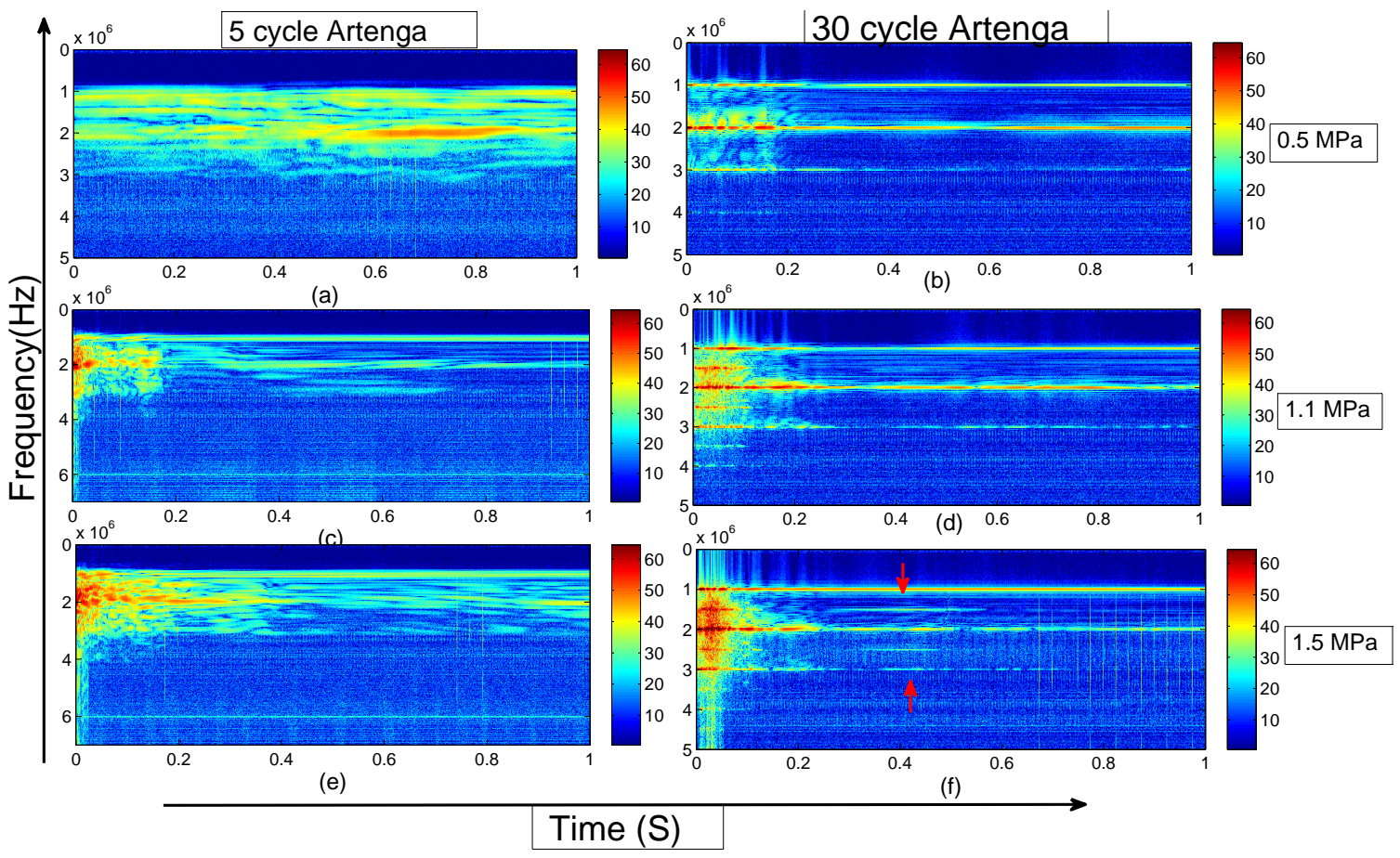

Figure 3.6: Spectrograms for ART MB, at 5 cycles pulse duration and PNP (0.5, 1.1, 1.5 MPa) for the signals received with $2.25 \mathrm{MHz}$ transducer. The vertical axis represents frequency $(\mathrm{Hz})$ and horizontal axis represents time $(\mathrm{s})$. The color bar represents the frequency spectral amplitude, with red the highest and blue the lowest.

while they are 13, 25, 3 and $12 \mathrm{~dB}$ for the ultraharmonic $(1.5 f)$, second harmonic $(2 f)$, ultraharmonic $(2.5 f)$ and third harmonic $(3 f)$ respectively at $1.5 \mathrm{MPa}$. In addition the spectrograms comparision between DEF and ART at 30 cycle (Figures 3.3, 3.7, B.1, B.2) have shown the frequency amplitude are greater for ART which support our results that ART have greater peak pressure amplitudes than DEF in FFT plots (Figure 3.5 and B.6)

The spectrogram (Figure 3.6) shows the time dynamic behaviour of the ART MBs for five cycles exposure, which were received with $2.25 \mathrm{MHz}$ transducer. It exhibits a wide range of frequency spectra distribution for first $200 \mathrm{~ms}$ of insonation time at 1.5 and 1.1 Mpa. At 0.5 Mpa, the frequency contents are of low magnitude and greatly varies 
with time. As insonation time increases, the spectra $(f, 2 f, 3 f)$ have very low frequency amplitude compared to that of 30 cycles results shown in Figure 3.3 (b, d, e). Similar results from experiment 2 and experiments 3 are shown in appendix (Figure B.3).

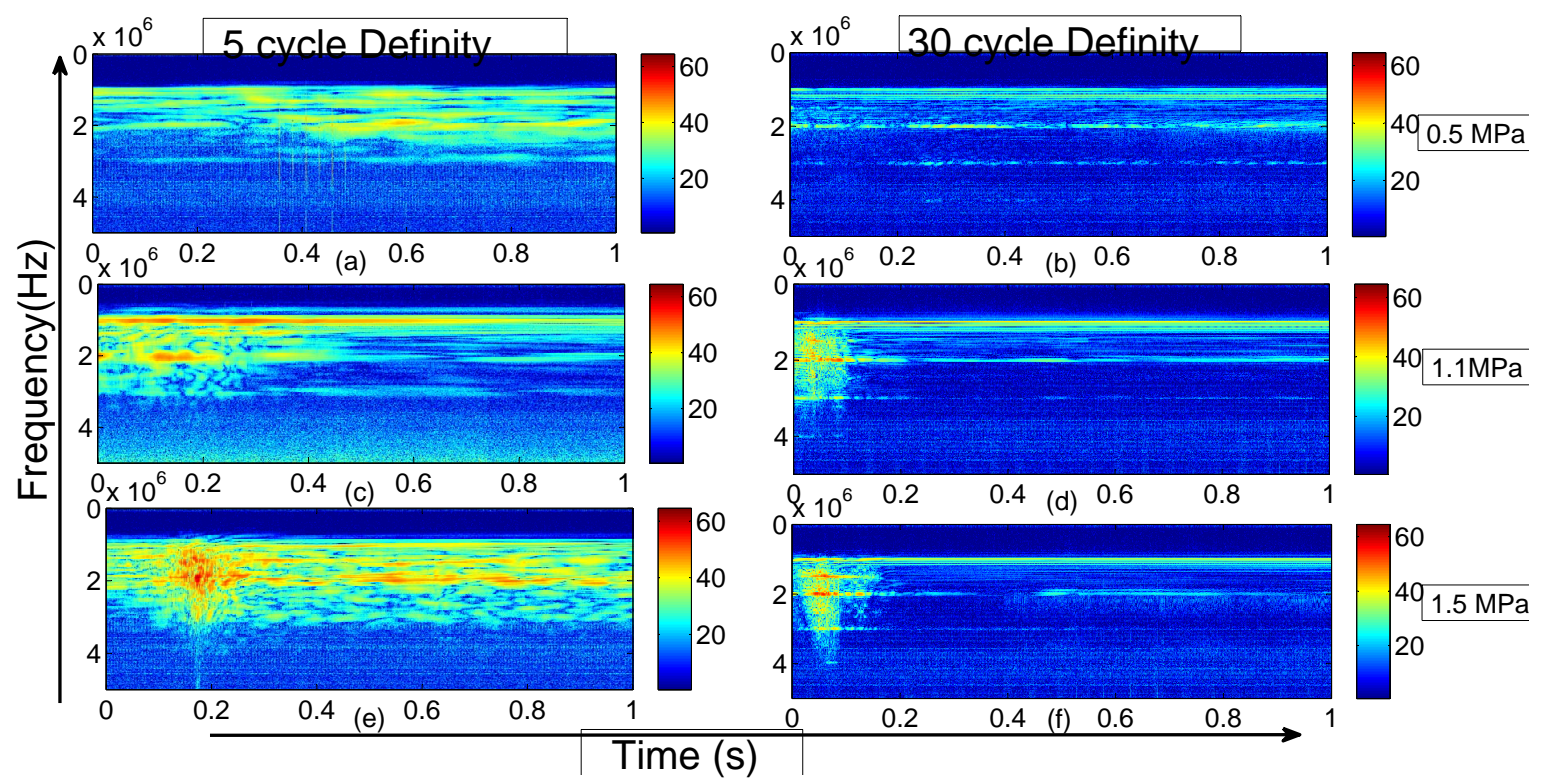

Figure 3.7: Spectrograms for DEF MB, at 5 and 30 cycles pulse duration and PNP ( 0.5, 1.1, 1.5) MPa for the data received with $2.25 \mathrm{MHz}$ transducer. The vertical axis represents frequency $(\mathrm{Hz})$ and horizontal axis represents time $(\mathrm{s})$. The color bar represents the frequency spectral amplitude, with red the highest and blue the lowest.

The time dynamics of frequency spectra for the DEF MB at 5 cycles and 30 cycles pulse duration are shown in Figure 3.7. The spectrograms demonstrate that the signal from the MBs when exposed to 5 cycles, have variable frequency amplitude for total exposure time of one second, with signals of higher magnitude at around $200 \mathrm{~ms}$ while the signals for 30 cycles pulse duration are observed with higher magnitude at 45 to 80 ms and there are no signals after $200 \mathrm{~ms}$ except $f$ and $2 f$ of small magnitude. Similar results from experiment 2 and experiment 3 are shown in appendix (Figure B.2 and B.4). 


\subsection{Cavitation Dose}

The cavitation dose plots (RMS FFT amplitude vs time) for ART MB at 30 cycles insonation pulse and PNP of 0.5, 1.1 and 1.5 MPa are shown in Figure 3.8. Cavitation doses are calculated at the desired frequency windows $(0.5 \pm 0.2,2 \pm 0.2,1.5 \pm 0.2,2.5 \pm 0.2$ $\mathrm{MHz})$ as the subharmonic cavitation dose, second harmonic cavitation dose and ultraharmonics cavitation dose respectively. Results demonstrate that the cavitation dose increases as a function of pressure. At $1.5 \mathrm{MPa}$, the subharmonic $(f / 2)$ cavitation dose is greater (40 units) at $5 \mathrm{~ms}$ and decreases significantly to 2 units at $100 \mathrm{~ms}$, following the lower values with increase of insonation time (Figure 3.8 (a)). The ultraharmonic (1.5f) cavitation dose has shown that it grows with insonation time, attains a maximum value of 43 units at $34 \mathrm{~ms}$ and decays slowly to 2 units at $120 \mathrm{~ms}$ (Figure 3.8 (b)). Further, the second harmonic cavitation dose plots have indicated that it grows to 74 units at $30 \mathrm{~ms}$ and decreases to 5 units at $200 \mathrm{~ms}$ (Figure 3.8 (c)). The ultraharmonic cavitation dose plot demonstrate that the cavitation dose suddenly grows higher at $10 \mathrm{~ms}$, maintains its amplitude up to $50 \mathrm{~ms}$ (average of 17 units) and finally decreases to 2 units at $120 \mathrm{~ms}$. Similar decrease of cavitatitation dose with increase of insonation time can be seen in Figure 3.8 at 1.1 and $0.5 \mathrm{MPa}$. The cavitation dose follows the similar trend of increase with PNP and decrease with insonation time for all others experimental results.Some

of the results are shown in appendix (Figure B.7 and B.8). The water signals (blue in colour) at $1.1 \mathrm{MPa}$ at each frequency window were shown as a reference signal.

Integrated cavitation doses for DEF and ART MBs at 30 cycle pulse duration are shown in Figures 3.9 and 3.10. The error bar represents the mean \pm standard deviation for signals from 3 experiments. The ICD increases with increase of PNP for each spectral feature. The subharmonic ICD is saturated for DEF MB at 1.1 MPa while large growth 

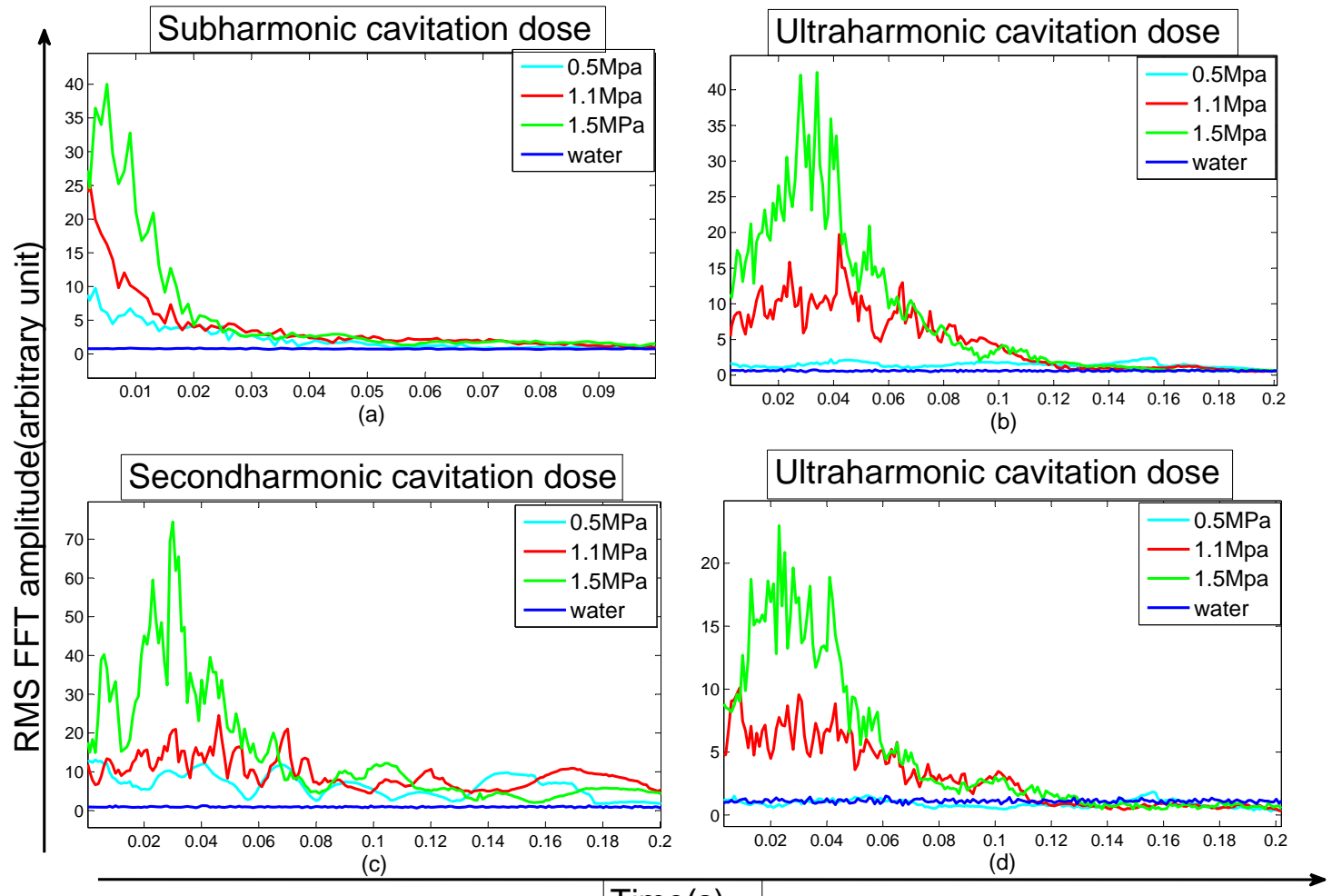

Time(s)

Figure 3.8: RMS FFT amplitude vs time plots for ART MB at 30 cycles pulse duration. (a), (b), ( c) and (d) represent the cavitation dose for subharmonic $(0.5 \pm 0.2 \mathrm{MHz})$, ultraharmonic $(1.5 \pm 0.2 \mathrm{MHz})$, harmonic $(2 \pm 0.2 \mathrm{MHz})$ and ultraharmonic $(2.5 \pm 0.2 \mathrm{MHz})$ respectively.The vertical axis represents the RMS FFT amplitude (arbitrary unit) and horizontal axis represents the time (s).

of subharmonic at 1.1 MPa can be seen for ART MB. In addition the ICD values at each pressures for subharmonics are greater for DEF MBs (Figure 3.9). The cavitation dose for ultraharmonic and second harmonic are greater for DEF MBs. The cavitation dose for DEF MBs are saturated at 1.1 MPa while strong growth of ultraharmonic cavitation dose for ART MBs is observed (Figure 3.10).

Integrated cavitation doses for $\mathrm{DEF}$ and $\mathrm{ART}$ MBs at 5 cycle pulse duration are shown in Figure 3.11 and Figure 3.12. The error bar represents the mean \pm standard deviation for signals from 3 experiments. The ICD increases with increase of PNP for 


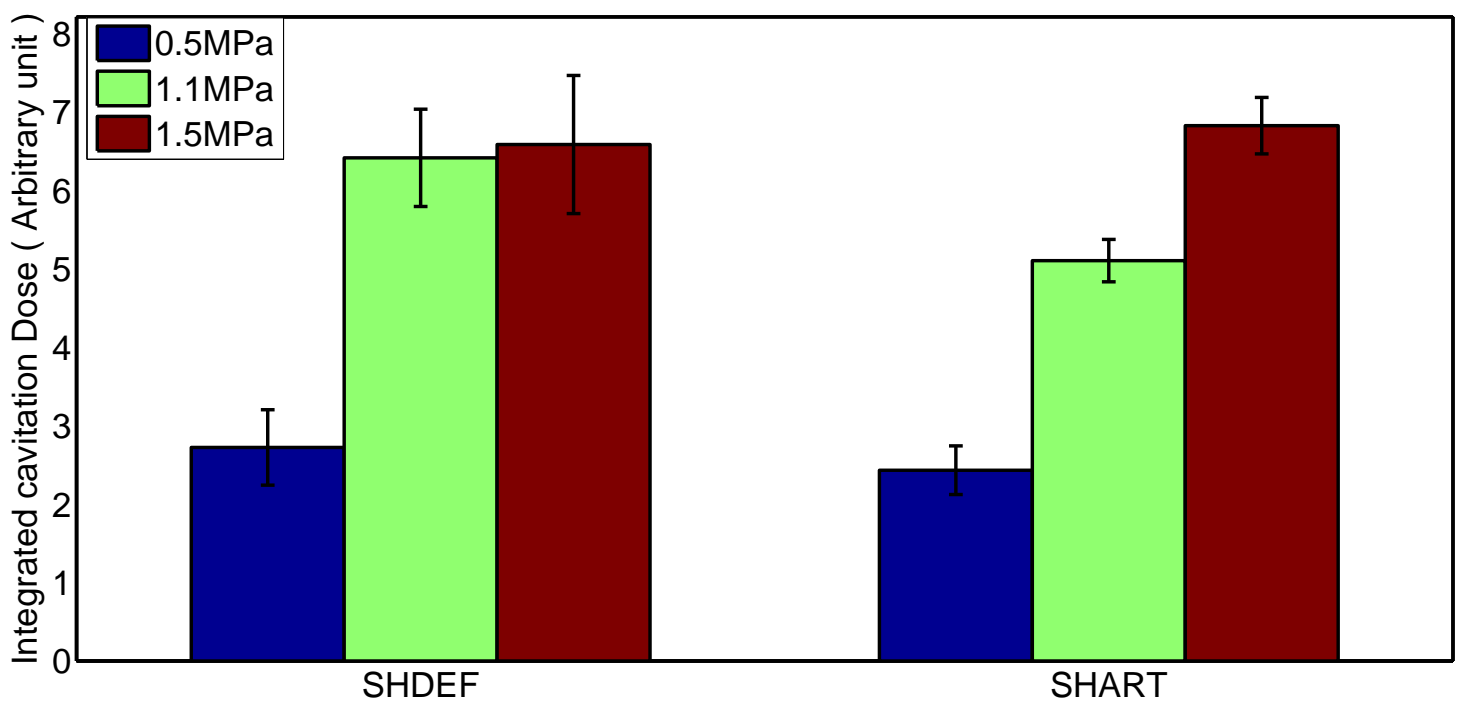

Figure 3.9: Integrated cavitation dose for DEF and ART MBs for subharmonic $(0.5 \pm 0.2$ $\mathrm{MHz}$ ) frequency spectrum for signals received with $0.5 \mathrm{MHz}$ transducer, at acoustic pressure of $0.5,1.1$ and $1.5 \mathrm{MPa}$ and 30 cycle pulse duration. The vertical axis represents the ICD (arbitrary unit) and horizontal axis represents subharmonic frequency spectra at different pressures. The error bar represents the mean \pm standard deviation for signals from 3 experimentals.

each spectral feature. The subharmonic ICD is close to saturation level for DEF MB at 1.1 MPa while large growth of subharmonic ICD is seen for ART MB between 1.1 and 1.5 MPa. In addition the ICD values at all pressures are greater for DEF MBs except at 1.5 MPa (Figure 3.11). The cavitation dose for ultraharmonic and second harmonic are greater for DEF MBs. The harmonic cavitation dose for DEF MBs is saturated at 1.1 MPa . The small growth of ultraharmonic cavitation dose for ART and DEF MBs is observed at 1.1 and $1.5 \mathrm{MPa}$ (Figure 3.12).

Results demonstrate that the integrated cavitation dose values are greater for DEF MB for most of the frequency components than corresponding frequency spectra for ART MB. This implies that the higher cavitation dose of DEF at 5 cycle pulse duration and 


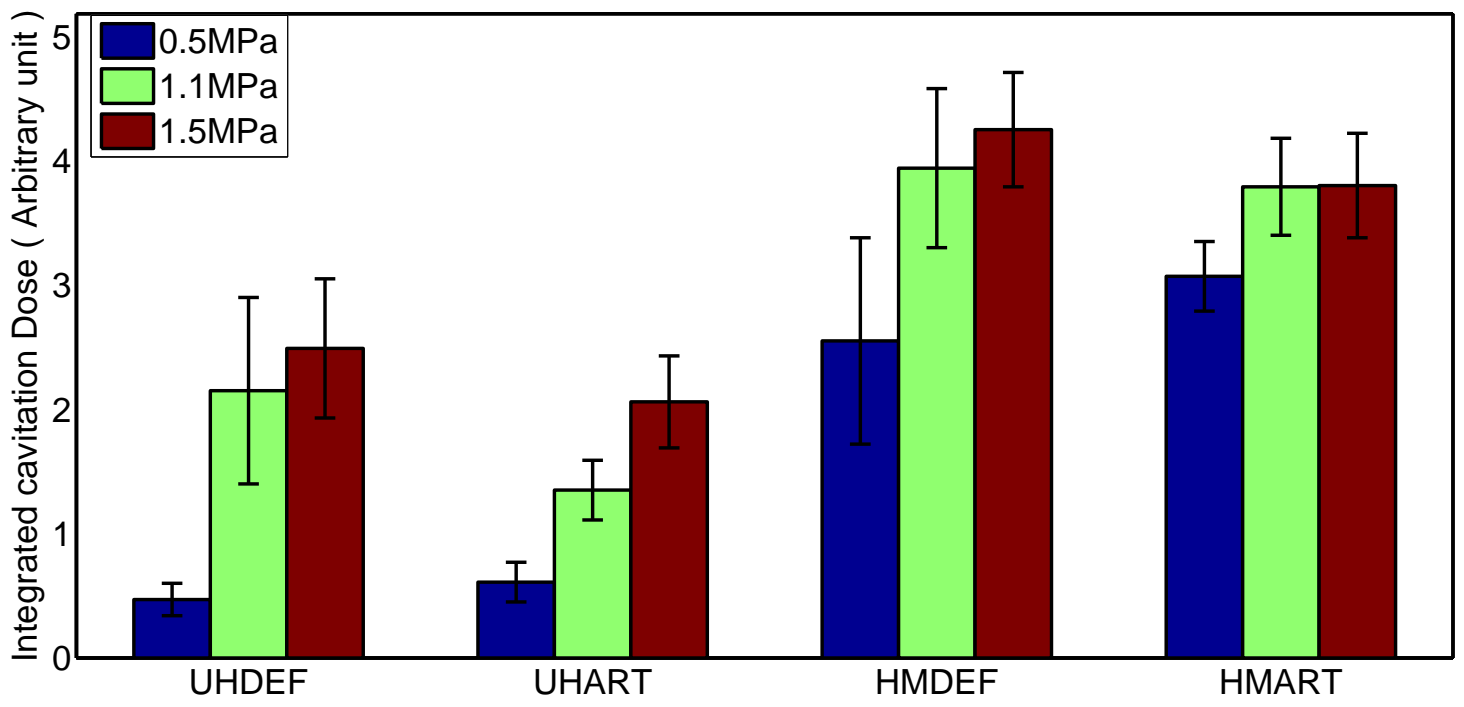

Figure 3.10: Integrated cavitation dose for DEF and ART MBs for ultraharmonic $(1.5 \pm 0.2 \mathrm{MHz})$ and second harmonic $(2 \pm 0.2 \mathrm{MHz})$ frequency spectra for signals received with $2.25 \mathrm{MHz}$ transducer, at acoustic pressure of $0.5,1.1$ and $1.5 \mathrm{MPa}$ and 30 cycle pulse duration. The vertical axis represents the ICD (arbitrary unit) and horizontal axis represents frequency spectra at different pressures.The error bar represents the mean \pm standard deviation for 3 experiments.

higher pressure may be useful for inducing bio-effects while higher and more pronounced acoustic response from ART MBs at 30 cycle pulse duration may be a good choice for MB activities monitoring purpose. 


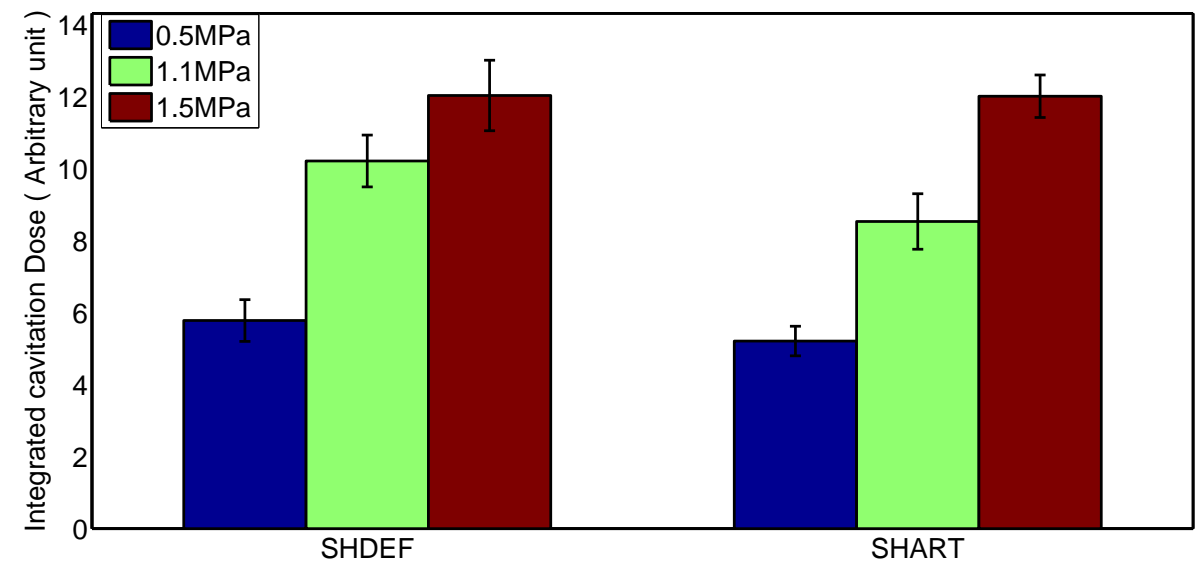

Figure 3.11: Integrated cavitation dose for DEF and ART MBs for subharmonic $(0.5 \pm 0.2$ $\mathrm{MHz}$ ) frequency spectrum for signal received with $0.5 \mathrm{MHz}$ transducer, at acoustic pressure of $0.5,1.1$ and $1.5 \mathrm{MPa}$ and 5 cycle pulse duration. The vertical axis represents the ICD (arbitrary unit) and horizontal axis represents frequency spectra at different pressures. The error bar represents the mean \pm standard deviation for 3 experiments.

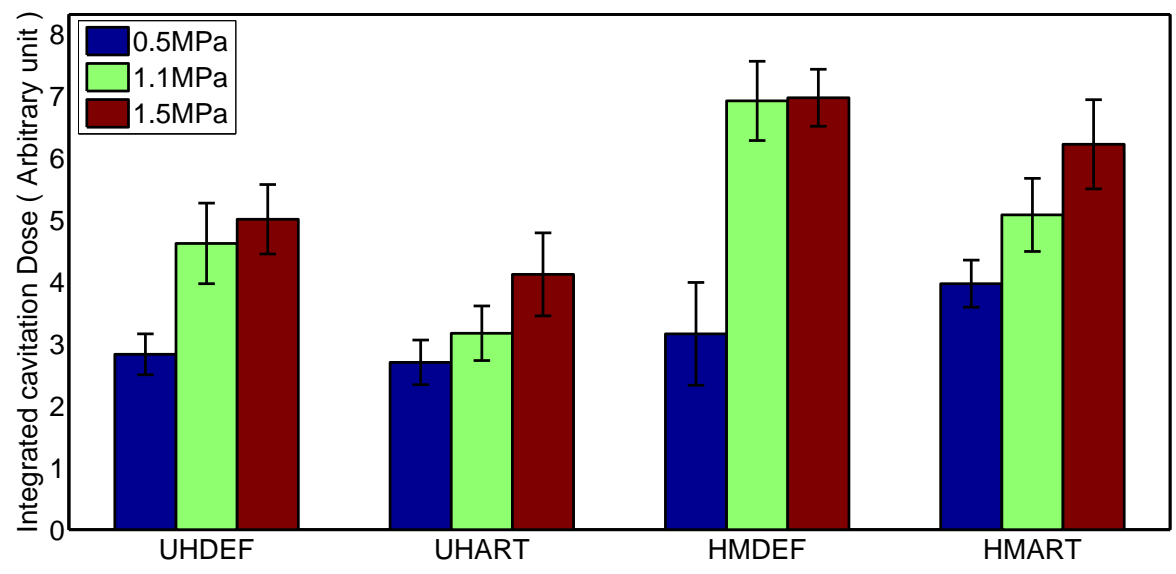

Figure 3.12: Integrated cavitation dose for DEF and ART MBs for ultraharmonic $(1.5 \pm 0.2 \mathrm{MHz})$ and second harmonic $(2 \pm 0.2 \mathrm{MHz})$ frequency spectra for signals received with $2.25 \mathrm{MHz}$ transducer, at acoustic pressure of $0.5,1.1$ and $1.5 \mathrm{MPa}$ and 5 cycle pulse duration. The vertical axis represents the ICD (arbitrary unit) and horizontal axis represents frequency spectra at different pressures.The error bar represents the mean \pm standard deviation for 3 experiments. 


\section{Chapter 4}

\section{Discussion}

The MB acoustic behaviours of DEF and ART were studied for various acoustic pressures and pulse durations using passive cavitation detection measurements. The time domain signals and their corresponding FFT spectra were analysed to characterise their acoustic response. Spectrogram analysis was employed to investigate their time dynamic response to ultrasound and a cavitation dose technique was used for their quantification.

\subsection{Nonlinear Oscillation}

Results have demonstrated that the nonlinear response of the MBs increases with the PNP. At $0 \mathrm{Mpa}$, the MB exhibited no sigh of oscillation (Figure 3.1) which demonstrated that certain pressure threshold is requided for its oscillation. The non-linear oscillation regime of the $\mathrm{MB}$ was identified for the acoustic pressure between 0.5 to $1.5 \mathrm{MPa}$. It was shown that the asymmetric, irregular and aperiodic oscillations of the MB increased with PNP, that emitted frequency spectra at the subharmonic, ultraharmonics, harmonics, and broadband spectra (Figures 3.1 and 3.2). Moreover, only above certain 
pressure thresholds, the characteristic markers of the nonlinear oscillation of MB such as sub-harmonics and ultra-harmonics are detected. These MBs characteristics frequency spectra (subharmonics and ultraharmonics) are believed to be associated with the bubble motion undergoing period doubling, period of four and period of eight as shown by using the bifurcation diagram analysis $(23)$.

We have shown that with pulse duration, the acoustic behaviour of MBs greatly vary. Five cycle pulse duration results had very broad and lower peak pressure amplitude spectra by 8-47 dB than 30 cycles pulse duration. This is likely due to reason that the wide range of MBs are oscillating emitting the broad frequency spectrum from a broadband pulse (5 cycle). The narrowband pulse (30 cycle) is more specific to certain sizes of MBs, due to its small bandwidth. In addition, the damping and attenuation is greater for 5 cycle pulse as more MBs are responding to ultrasound pulse which cause the peak pressure amplitude are lower for 5 cycle pulse than that of 30 cycle pulse. It was observed that the ultraharmonic frequency spectrum (1.5f) appear at $0.5 \mathrm{MPa}$ for the 5 cycle pulse duration (Figures 3.5 and B.6) and spectrograms (Figures 3.6, 3.7, B.1, B.2, B.3 and B.4) while there are no such signal for 30 cycle pulse duration. Moreover, the FFT response (Figures 3.5 and B.6) agree with the results shown by (45) where the effect of pulse duration on emissions of sub-harmonic spectra was investigated and has shown that higher spectral emission (subharmonic) was achieved for higher pulse duration. These results have demonstrated that the choice of 30 cycle pulse duration is better for the monitoring purpose, as the FFT signals for 30 cycles are very high and clear from noise level.

The effect of acoustic pressure and pulse duration on MB acoustic response has shown the FFT spectra for ART are wider while spectra for DEF are narrower. This could possibly happen because of difference in encapsulation (elasticity and viscosity), sizes 
and gas contents which may results different scattered strength.

Spectrogram results have shown the time dynamics of the MB frequency spectra. The strong MB non linear activities at 30 cycle pulse duration are detected for $200 \mathrm{~ms}$ and no signals except fundamental and second harmonic are seen for longer insonation time (Figure 3.3, 3.7, B.1 and B.2).The subsequent decrease in spectral signals after $200 \mathrm{~ms}$ is believed to indicate the loss of bubbles that are destroyed or loss of stability of MB due to diffusion of the gas, which reduce both scattering and emissions from MBs. Thus, fewer bubbles may not be efficient enough to show the significant bubble response. This provides us an idea about the life span of MB during insonation.

Spectrograms (Figures 3.6 and 3.7) demonstrate that the pulse duration effects the MB behaviours, i.e the broadband frequency spectra showing the MBs disruption phenomena at some time points were distinctly observed for 5 cycle pulse duration compared to 30 cycles pulse duration (Figures 3.6, 3.7, B.3 and B.4). In addition the MBs activities persisted for longer insonation time for 5 cycle pulse duration, which is the result of MBs oscillating continuously for longer period of time before get disrupted.

We have shown that there is significant difference in the strength of MB frequency spectral emissions and their time dynamics for the DEF (FDA approved) and ART (clinical trial) agents (Figures 3.6 and 3.7). The frequency spectra amplitude was higher for ART MB for first $200 \mathrm{~ms}$ while they delayed by 70 to $200 \mathrm{~ms}$ in case of DEF MBs. In addition, it is shown that DEF oscillates for longer time and get dusrupted, while ART have shown oscillation at the beginning of insonation time points and persists for almost $200 \mathrm{~ms}$ for 5 cycle pulse duration. This could possibly happen because of difference in encapsulation (elasticity and viscosity), sizes and gas contents which may results different scattered strength and at different time points. Similar information can be seen in appendix Figures B.3 and B.4. 


\subsection{Cavitation dose}

The cavitation dose (Figures 3.8, B.7, B.8, B.9, B.10) and integrated cavitation dose (Figures 3.9, 3.10, 3.11, 3.12) for each of the frequency content (subharmonic, second harmonic and ultraharmonics) increases with pressure which agrees with the published results shown by $(57 ; 56 ; 39)$ where the integrated cavitation dose in the certain frequency window for broadband emissions were investigated and have shown that it increases with the pressures. As the pressure increases the MBs oscillates nonlinearly in large scale and at same time large numbers of the MBs get disrupted emitting the sound which give rise to higher integrated cavitation dose. The integrated cavitation dose was found saturated for second harmonic at $1.1 \mathrm{MPa}$ which is due to the energy redistribution to the ultraharmonics spectra.

The integrated cavitation dose was obtained greater for 5 cycles pulse duration which contradicts the results of $(60)$ where they have shown that with increase of pulse length integrated cavitation dose was higher. This could have occurred because it has been shown that the integrated cavitation dose for broadband emissions associated with the MBs destruction phenomenons was investigated by (60) while in our experiments the investigation was on the non-linear regimes where most of the bubbles undergoes nonlinear oscillating rather than destruction. The reason of having higher integrated cavitation dose at 5 cycles is that the spectra are broader at 5 cycles which content more acoustic energy, which was emitted from wide range of MBs. The longer MB activities seen in spectrogram at 5 cycles pulse duration support the results of having higher integrated cavitation dose. The cavitation dose was greater for DEF MBs than that of ART which possibly be the concentration and size effects which give more MB activities as seen for longer time as demonstrated in Figures(3.6, 3.7, B.3, B.4). This suggest higher number 
density of Definity with smaller sizes may oscillates at large scale compared to Artenga MB and thus give higher integrated cavitation dose.Therefore, Definity might be a right choice for inducing bioeffects because it has been shown that higher the cavitation dose more is the induced bioeffects.

Finally this work suggests two different future directions: In order to develop frequency spectra as monitoring tools, we require high amplitude acoustic spectra, that was achieved at intermediate (1.1 MPa) acoustic pressure and higher (30 cycle) pulse duration and for ART MBs while the higher integrated cavitation dose might correlates to enhance permeability which can improve sonoporation applications that was obtained at higher pressure but lower pulse duration and was greater for DEF MBs.

\subsection{Limitations of this study}

The MB preperation and conducting experiments for long hours was challanging. Using MBs suspension at time of generation and after half an hour make a difference of few $\mathrm{dB}$ in emissions spectrum which may influence the results of peak amplitude of frequency spectra. PCD experiments were performed throughout the period of two years and ecah

time the MB viais used in the experiements were different. Since the MB vial kept for longer time from date of manufacture have shown weaker response compared to the fresh MBs, this might have influence on our results. Moreover the PCD experiments were conducted in water at room temperature which may not represent the complexity of the vivo tissue models. In addition, the water was used as a reference which cannot account for nonlinear propogation effects on MBs response. The bandwidth of the receiver trnsducers was not taken into account during the analysis of PCD data. 


\subsection{Possible applications and clinical relevance}

Improving our nderstanding of MB behaviors and correlating them with induced bioeffects may enable us to increase efficacy of ultrasound and MB mediated applications. This project has potentially open a way of enhancing sonoporation activities and monitoring it based on the MB acoustic characterization. The acoustic methods developed in bench top system can be translated and tested in vivo models. 


\section{Chapter 5}

\section{Conclusion and Future work}

\subsection{Conclusion}

The non-linear oscillation of MBs increases with acoustic pressure and pulse duration. MBs exhibits strong subharmonics, and ultraharmonics at $1.1 \mathrm{MPa}$ at 30 cycle pulse duration while and at $0.5 \mathrm{MPa}$ for 5 cycle pulse duration. MBs activities (oscillation and disruption) appear significant up to $200 \mathrm{~ms}$ for 30 cycle pulse duration, and appear longer for 5 cycle pulse duration. The integrated cavitation dose increased with acoustic pressure for all frequency components including subharmonic, ultraharmonics and second harmonic, and decreases with pulse duration. The ICD was greater for DEF MBs compared to ART MBs.

\subsection{Future work: Effect of Microbubbles Size}

In this section, the acoustic characteristics of the filtered DEF MBs exposed at varying acoustic pressure (0.5-1.5 MPa), pulse duration $30 \mu$ s and different MB sizes for 
insonation time of 1 second was investigated under the same exposure conditions of PCD experiments that was described in chapter two. Methods includes the MBs size isolation through centrifuge which was performed based on the migration of MB in the centrifugal field(61). The MB suspension was centrifuged and MBs were acquired from different depths of a tube corresponding to small and large bubbles. Large MBs were at the top while smaller ones were at the bottom of the tube. Analysis of data was perfoemed for ectracting spectrogram and cavitation dose methods. Spectrogram results are shown in Figures 5.1 and 5.2 and integrated cavitation dose in figure 5.3

\subsubsection{Results and discussion}

The acoustic behaviours of filtered DEF MBs exposed to 30 cycles pulse at acoustic pressure of $0.5,1.1,1.5 \mathrm{MPa}$ and measured with $2.25 \mathrm{MHz}$ transducer are shown in spectrograms Figure 5.1. The MBs exhibit different acoustic responses depending on size. Results demonstrate that the smaller size MBs exhibit higher frequency amplitude than the larger sizes. The time dynamic response is variable (appears and disappears) for filtered MBs within insonation time of $1 \mathrm{~s}$ while it is less than $100 \mathrm{~ms}$ for unfiltered MB and after that no signals are seen except fundamental and second harmonic (Figure $5.1)$.

The signals are received with $0.5 \mathrm{MHz}$ transducer, where we are interested at subharmonic frequency response. At $0.5 \mathrm{Mpa}$, the frequency amplitude for subharmonic spectrum was lower for large MBs compared to smaller one while at $1.1 \mathrm{MPa}$ it was greater than for larger MBs. At 1.5 MPa the frequency amplitude for subharmonic was greater for the smaller MBs for $200 \mathrm{~ms}$ and slowly decays.

The ICD are plotted for the subharmonic $(0.5 \pm 0.2 \mathrm{MHz})$, ultraharmonic $(1.5 \pm 0.2$ 


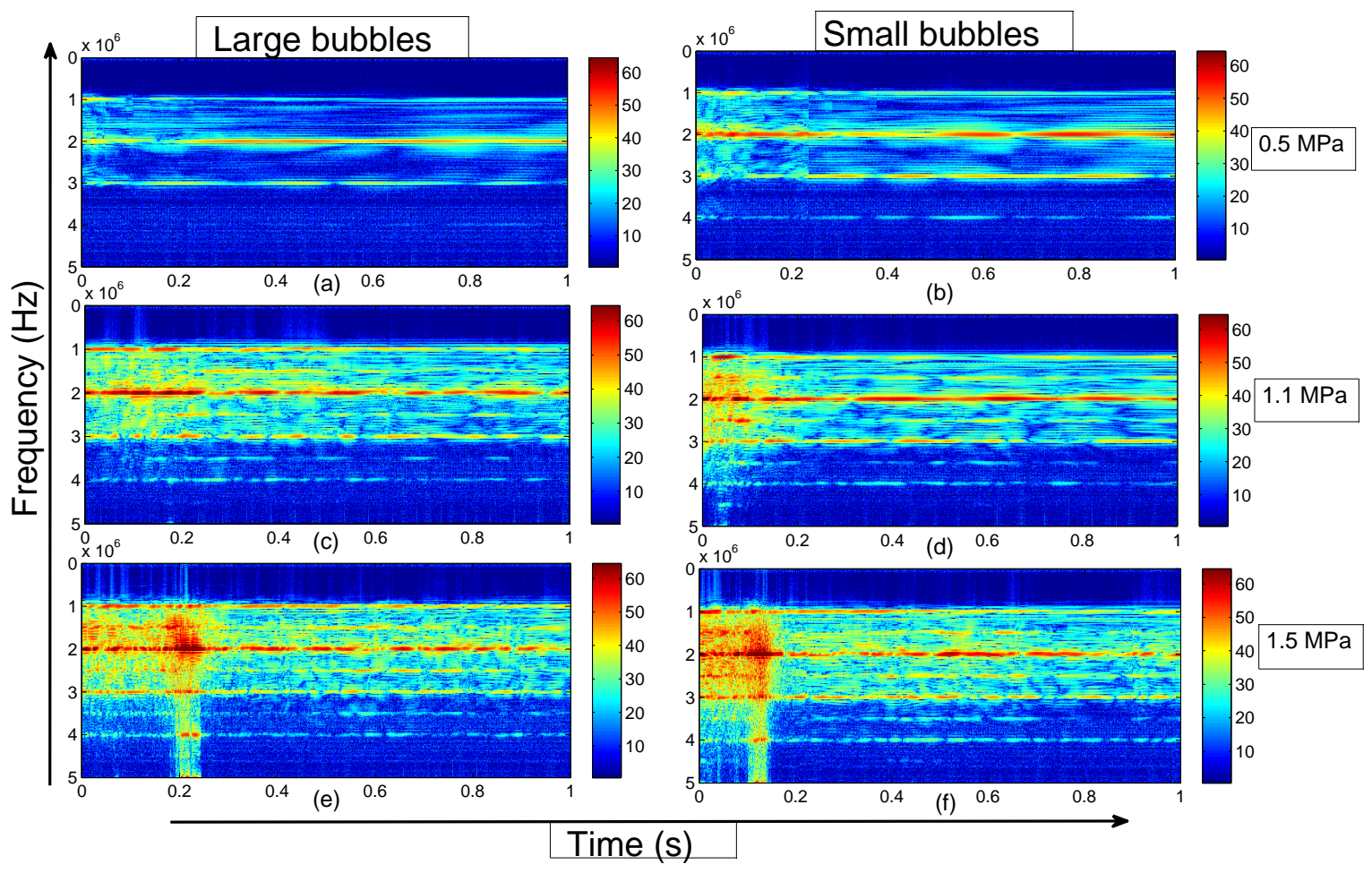

Figure 5.1: Spectrograms for filtered DEF MBs, at 30 cycle pulse duration and PNP $(0.5,1.1,1.5 \mathrm{MPa})$ for the signals received with $2.25 \mathrm{MHz}$ transducer. The vertical axis represents frequency $(\mathrm{Hz})$ and horizontal axis represents time $(\mathrm{s})$. The color bar represents the frequency spectral amplitude, with red the highest and blue the lowest.

$\mathrm{MHz})$, and second harmonics $(2 \pm 0.2 \mathrm{MHz})$ as a function of acoustic pressure and $\mathrm{MB}$ sizes (Figure5.5). It was observed that the cavitation dose for all frequency components increases with acoustic pressure. Large scale subharmonic, ultraharmonic and second harmonic growth is observed at 1.1 and $1.5 \mathrm{MPa}$ for large, small and unfiltered MBs. The ICD growth is relatively greater for larger MBs at 1.1 MPa while it is greater for small MB for all others exposure pressures. Moreover the ICD for second harmonics are greater than subharmonics followed by ultraharmonics.

Spectrograms have shown that the time dynamics greatly varies for different bubbles sizes. Smaller the MBs higher are the spectral amplitude and persisted for longer in- 


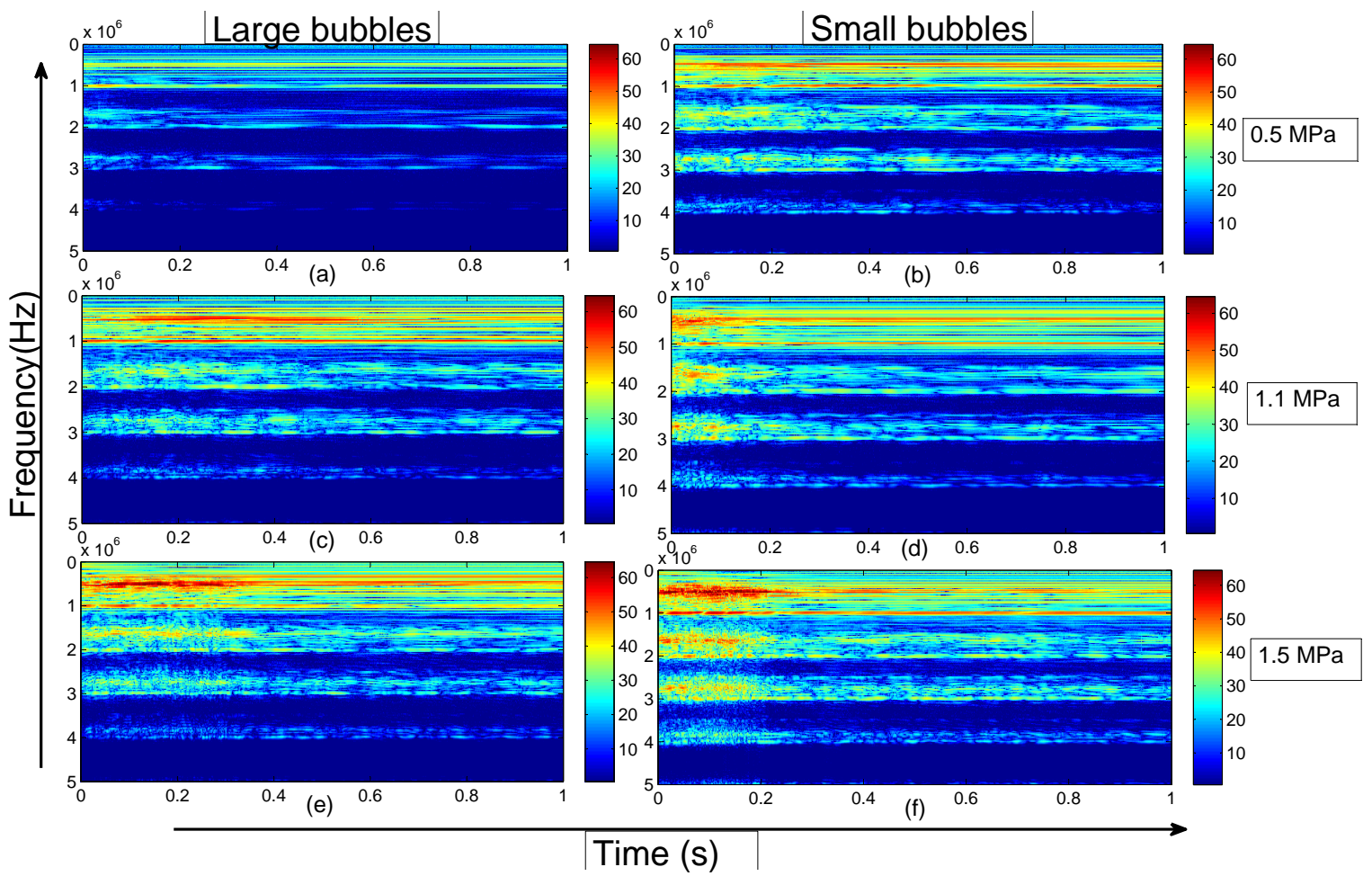

Figure 5.2: Spectrograms for filtered DEF MB, at 30 cycle pulse duration and PNP (0.5, 1.1, 1.5 MPa) for the signals received with $0.5 \mathrm{MHz}$ transducer. The vertical axis represents frequency $(\mathrm{Hz})$ and horizontal axis represents time $(\mathrm{s})$. The color bar represents the frequency spectral amplitude, with red the highest and blue the lowest.

sonation time (Figure 5.1). The integrated cavitation dose increases with PNP and was higher for smaller microbubbles for most of pressures compared to large and unfiltered MBs.

The influencing factors may be the stiffness of the MBs and surface tension which affects smaller microbubbles, while the larger MBs are inertia controlled. In addition, the number density is high for smaller MBs compared to larger bubbles which might produce more scattering and higher integrated cavitation dose.

To sum up, this future work results has shown that the size isolation is very important for understanding various MB behavior and their quantification that could measure 


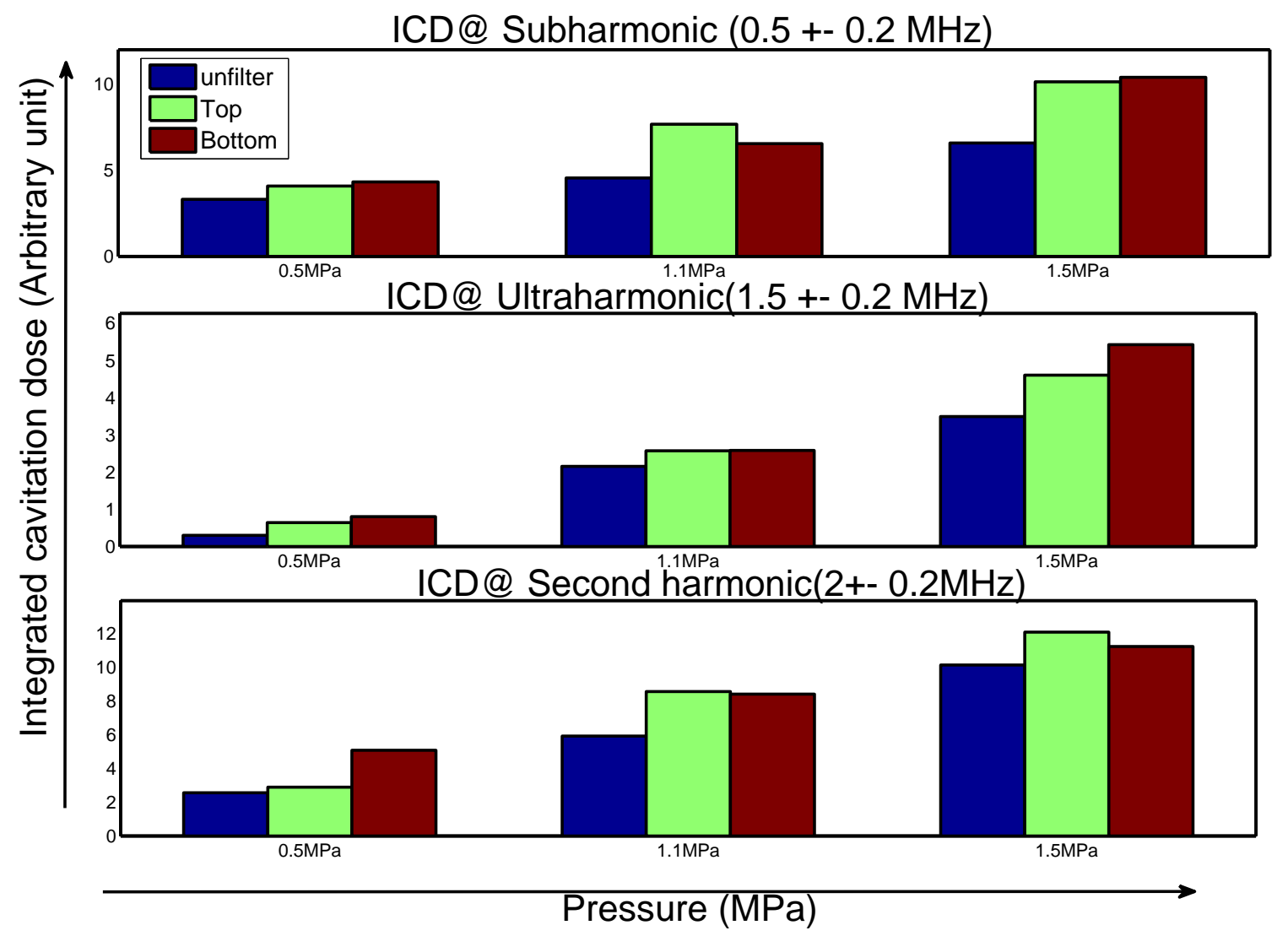

Figure 5.3: Integrated cavitation dose for DEF MBs at 30 cycle pulse duration and acoustic pressure of $0.5,1.1,1.5 \mathrm{MPa}$. PLots represents the cavitation dose for subharmonic $(0.5 \pm 0.2 \mathrm{MHz})$, ultraharmonic $(1.5 \pm 0.2 \mathrm{MHz})$, and harmonic $(2 \pm 0.2 \mathrm{MHz})$ for unfiltered, (blue) large (green) and small bubbles (red) respectively. The vertical axis represents the RMS FFT amplitude (arbitrary unit) and horizontal axis represents the time $(\mathrm{s})$.

sonoporation outcomes at narrow size distribution. The conclusive results for MB acoustic behaviours at narrow size distribution could be achieved estimating the resonance bubble sizes for insonation frequency and isolate bubble sizes below and above that size. More experiments should be performed at same conditions to verify these results. Finally, future work should correlate the integrated cavitation dose with cell permeability and viability. 
Appendices 


\section{Appendix A}

\section{Time domain signal}

The linear oscillation and nonlinear oscillation of the MBs are distinguished based on the frequency contents on the FFT plots. The linear oscillation exhibits fundamental $(f)$ and second harmonics $(2 f)$ while the nonlinear oscillation exhibits subharmonic $(f / 2)$, ultraharmonic $(3 f / 2,5 f / 2)$ and higher harmonics.

In term of oscillation of MBs (time domain signals) it has been mentioned that the symmetric, regular and periodic oscillation give rise to linear oscillation while asymmetric, irregular and aperiodic oscillation results nonlinear oscillation of MBs. Our results demonstrated that there is a nonlinear regimes at 0.5 to $1.5 \mathrm{MPa}$ where different patterns

of the oscillation from MBs can be seen Figure 3.1 and 3.2. The portion of the TD signal at $30-40 \mu$ s, for Figure 3.1 is shown in Figure A.1.

At $0.5 \mathrm{MPa}$, every $\mu$ s (e.g $31,32,33)$ repetitions of the one kind of oscillation pattern was seen while another kind of oscillation pattern was reported every $\mu$ s ( e.g 31.5, $32.5,33.5)$. The difference between two patterns was of 0.05 units in average toward the expansion while it was 0.11 units at contraction side which is an example of asymmetric oscillation. We define this as weak nonlinear oscillation where the fundamental, second 


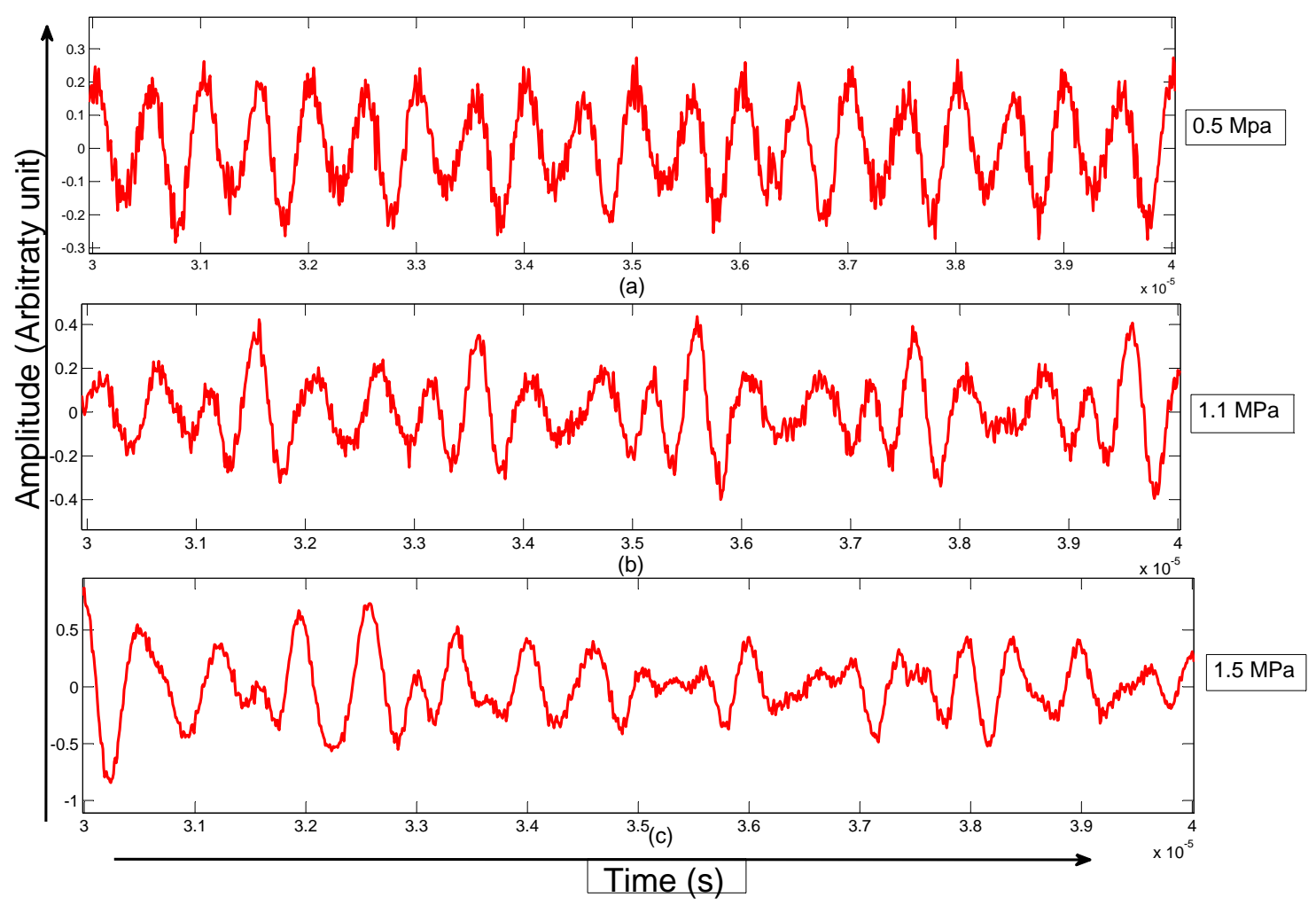

Figure A.1: Portion of the TD signal at $30-40 \mu \mathrm{s}$, at a pressure of $0.5,1.1$ and $1.5 \mathrm{MPa}$ shown by a, b, and c respectively. The vertical axis represent amplitude (Arbitrary unit) and horizontal axis represent time (s).

harmonic and third harmonics were emitted shown in Figure 3.1. At 1.1 MPa, repetition of the patterns of oscillation was seen every $2 \mu$ s (e.g 31.5, 33.5) with three small oscillation in a pattern, of lower amplitude in between which represents very strong nonlinear oscillation with an emissions of additional ultraharmonics, second and third harmonics of higher magnitudes. At 1.5 MPa the oscillation is very irregular, aperiodic and asymmetric which is difficult to predict its behaviour. This is called chaotic oscillation and its frequency content consists of broadband emissions.

Figure A.2 demonstrated that at $1.1 \mathrm{MPa}$, the MBs show expansions like behaviour where the average amplitude for expansion was greater by 0.12 units than the average 


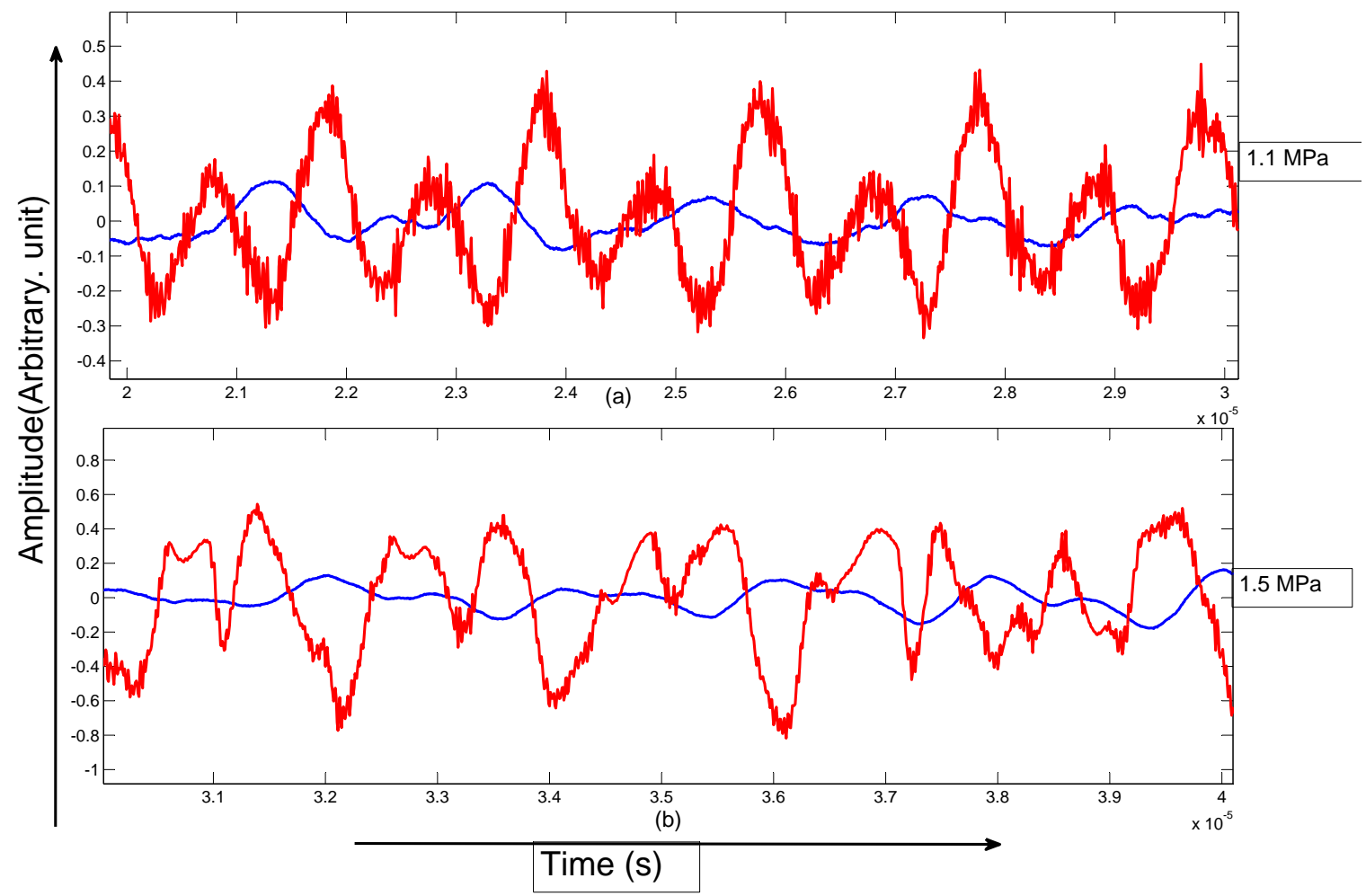

Figure A.2: Portion of the TD signal for $10 \mu \mathrm{s}$, at a pressure of 1.1 and $1.5 \mathrm{MPa}$ shown by $\mathrm{a}$, and $\mathrm{b}$ respectively. The vertical axis represent amplitude (Arbitrary unit) and horizontal axis represent time (s).

amplitude for contractions. At 1.5 MPa, the MBs show contraction like behaviour where the average amplitude for contraction was greater by 0.25 units than the average amplitude for expansion. This kind of behaviours are described by $(62)$ following the bubbles buckling and ruptured regimes as described by (29). It was mentioned at low amplitude, the compression -only behaviour was attributed to a reversible buckling of the lipid shell while the expansion only behaviours are attributed to rupturing regimes. 


\section{Appendix B}

\section{Experiments Results}

The PCD experiments results for ART and DEF MBs at PNP of 0, 1.1 and 1.5 MPa and 30 cycle pulse duration received with $2.25 \mathrm{MHz}$ transducer are shown in Figures (B.1 and B.2). Similar results for ART and DEF MBs at PNP of 0, 1.1 and 1.5 MPa and 5 cycle pulse duration received with $2.25 \mathrm{MHz}$ are shown in Figures (B.3 and B.4). In addition the spectrograms received with $0.5 \mathrm{MHz}$ transducers for DEF and ART MBs for 5 cycle pulse duration are shown in Figure B.5. The FFT spectra from second set of experiment for both MBs are shown in Figure B.6. Moreover the results for cavitation dose from second set of experiments are shown in Figures (B.7,B.8, B.9 and B.10). These results have demonstrated similar behaviours of microbubbles that are discussed in result chapters and support the conclusion drawn in this projects. 


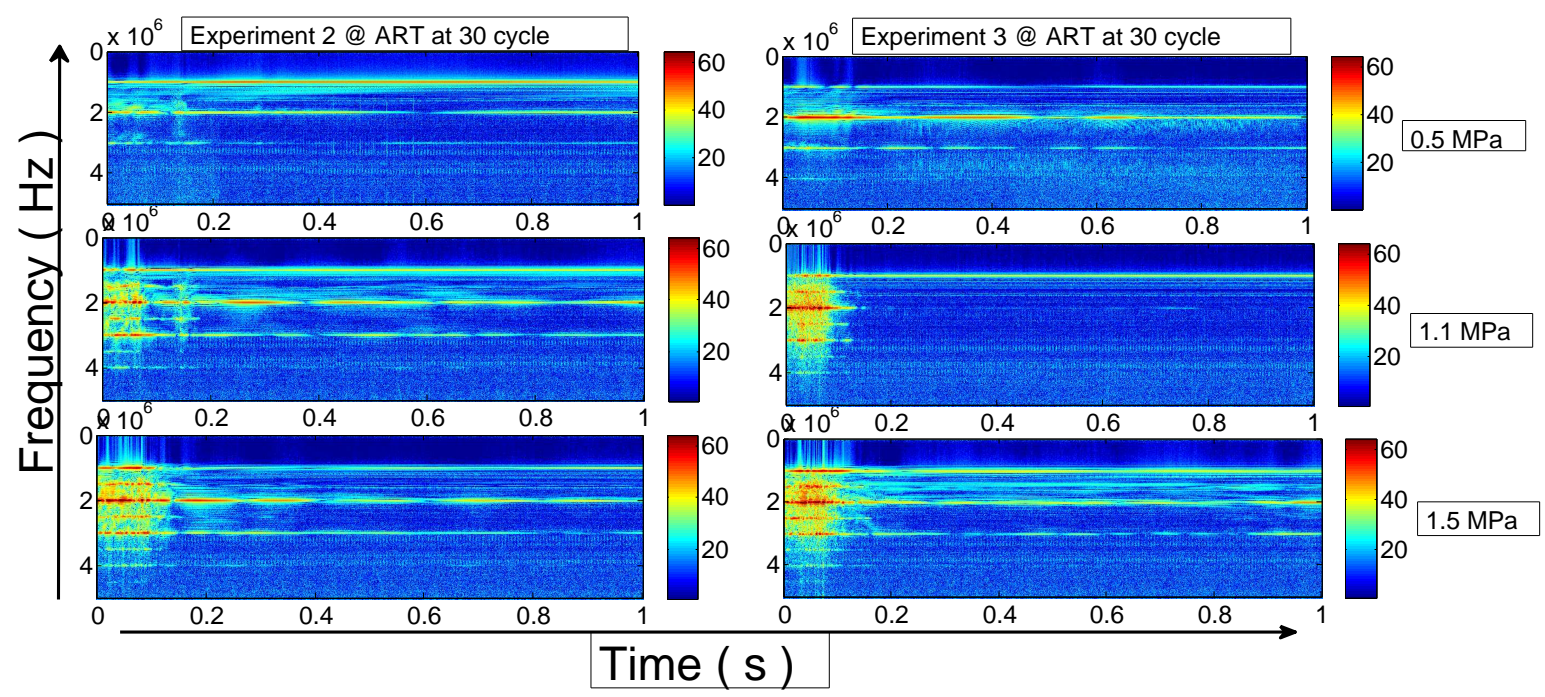

Figure B.1: ART MB spectrograms as a function of PNP for signals received with 2.25 $\mathrm{MHz}$ receiver transducer. Results of two different experiments are shown for $0.5,1.1,1.5$ $\mathrm{MPa}$ at 30 cycle pulse duration. The vertical axis on spectrogram represents frequency $(\mathrm{Hz})$ and horizontal axis represents time $(\mathrm{s})$. The color bar represents the frequency spectral amplitude, with red the highest and blue the lowest.

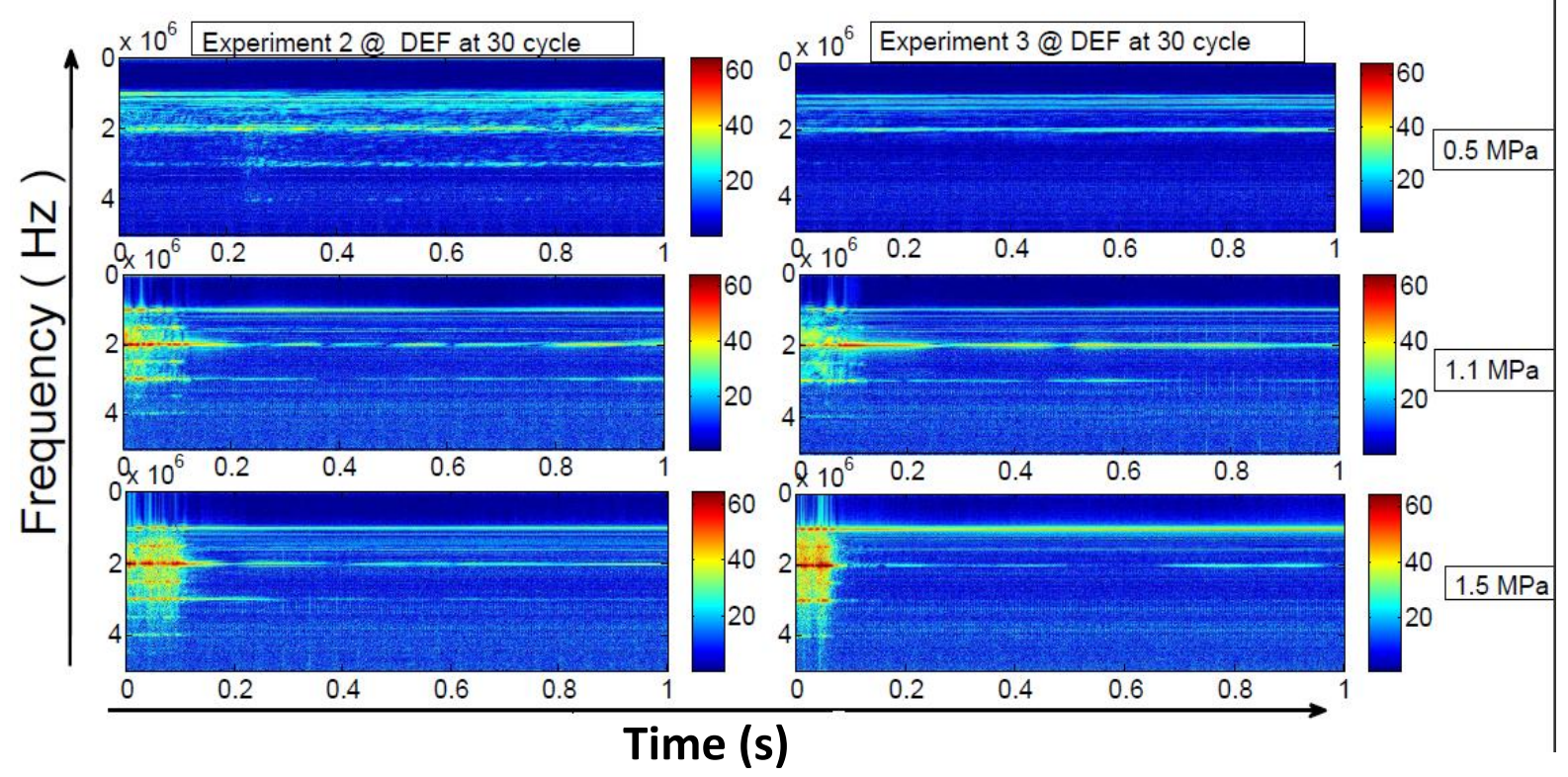

Figure B.2: DEF MB spectrograms as a function of PNP for signals received with 2.25 $\mathrm{MHz}$ receiver transducer. Results of two different experiments are shown for $0.5,1.1,1.5$ $\mathrm{MPa}$ at 30 cycle pulse duration. The vertical axis on spectrogram represents frequency $(\mathrm{Hz})$ and horizontal axis represents time (s). The color bar represents the frequency spectral amplitude, with red the highest and blue the lowest. 


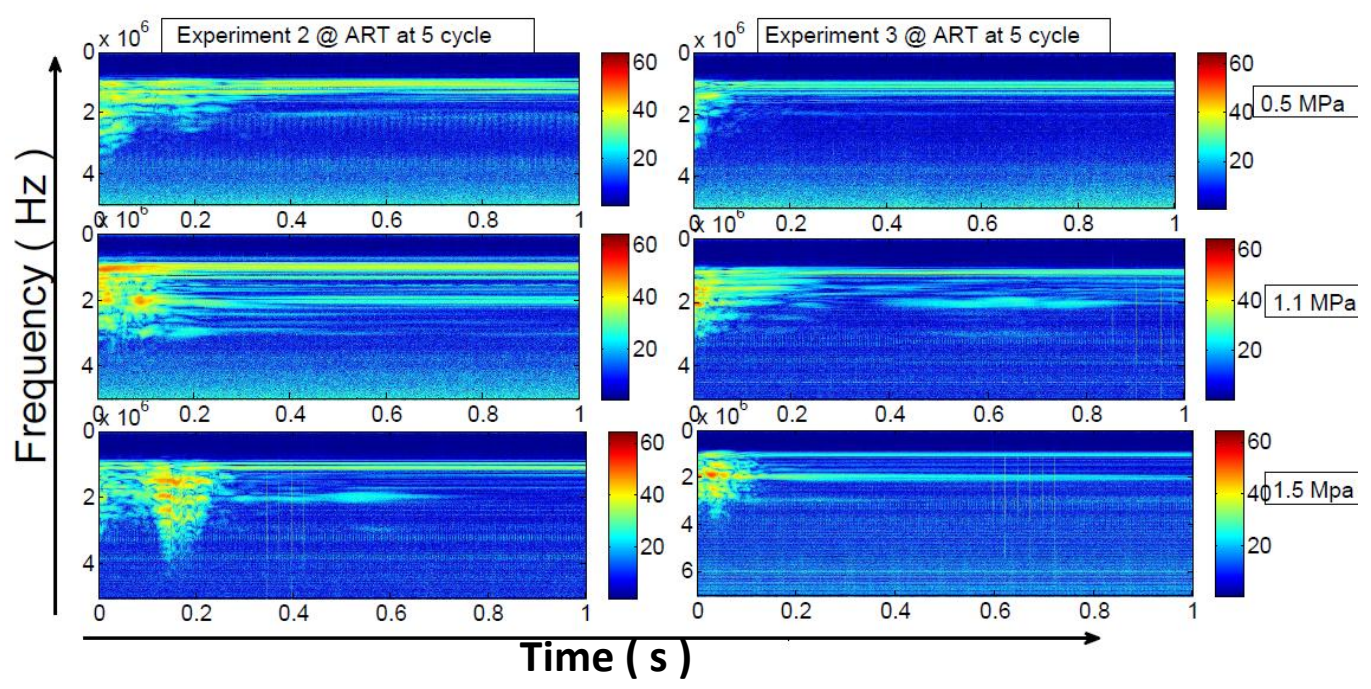

Figure B.3: ART MB spectrograms as a function of PNP for signals received with 2.25 $\mathrm{MHz}$ receiver transducer. Results of two different experiments are shown for $0.5,1.1,1.5$ $\mathrm{MPa}$ at 5 cycle pulse duration. The vertical axis on spectrogram represents frequency $(\mathrm{Hz})$ and horizontal axis represents time $(\mathrm{s})$. The color bar represents the frequency spectral amplitude, with red the highest and blue the lowest.

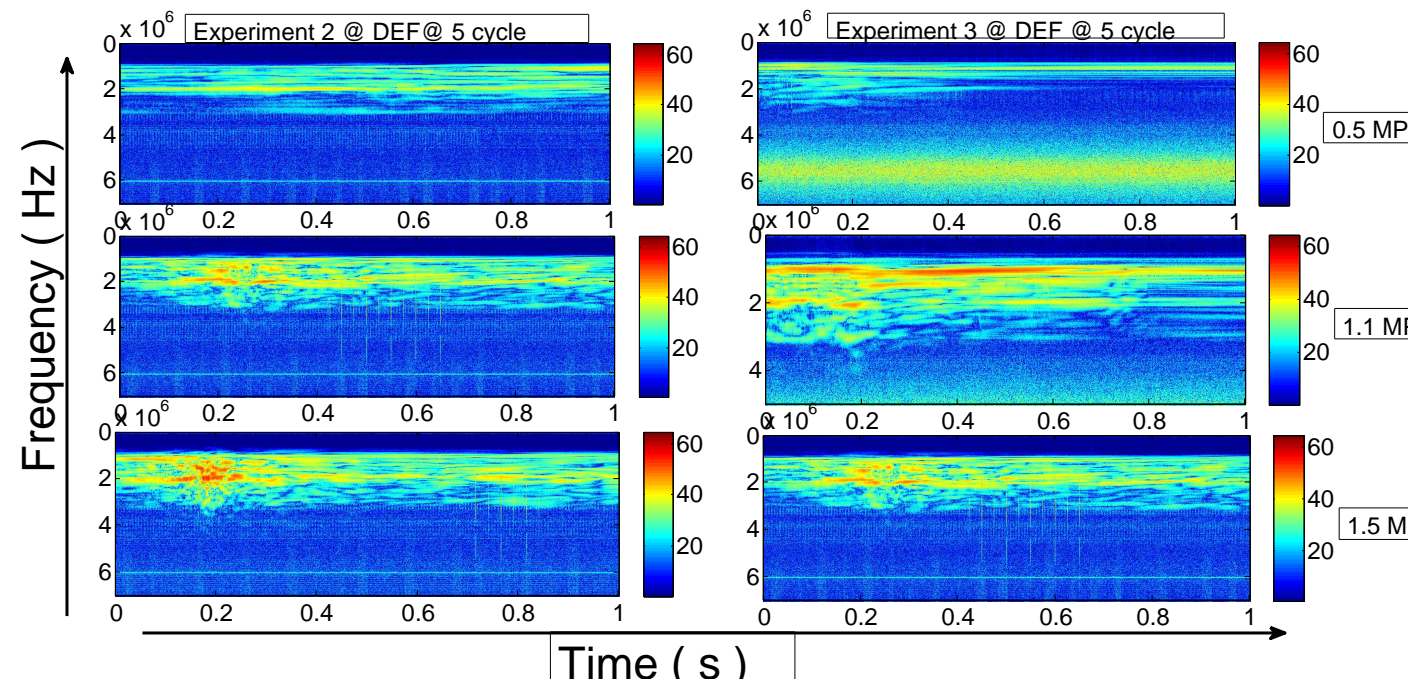

Figure B.4: DEF MB spectrograms as a function of PNP for signals received with 2.25 $\mathrm{MHz}$ receiver transducer. Results of two different experiments are shown for $0.5,1.1,1.5$ $\mathrm{MPa}$ at 5 cycle pulse duration. The vertical axis on spectrogram represents frequency $(\mathrm{Hz})$ and horizontal axis represents time (s). The color bar represents the frequency spectral amplitude, with red the highest and blue the lowest. 


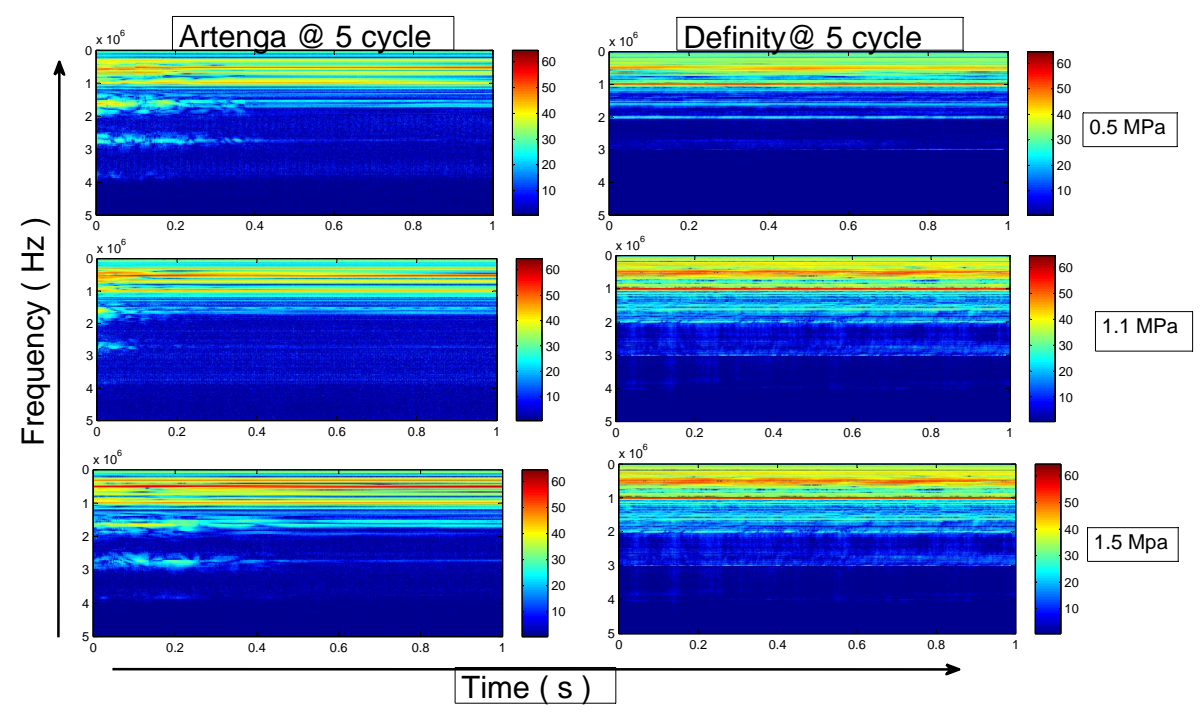

Figure B.5: ART and DEF MBs spectrograms as a function of PNP for signals received with $0.5 \mathrm{MHz}$ receiver transducer. Results of two different bubbles are shown for $0.5,1.1,1.5 \mathrm{MPa}$ at 5 cycle pulse duration. The vertical axis on spectrogram represents frequency $(\mathrm{Hz})$ and horizontal axis represents time (s). The color bar represents the frequency spectral amplitude, with red the highest and blue the lowest.

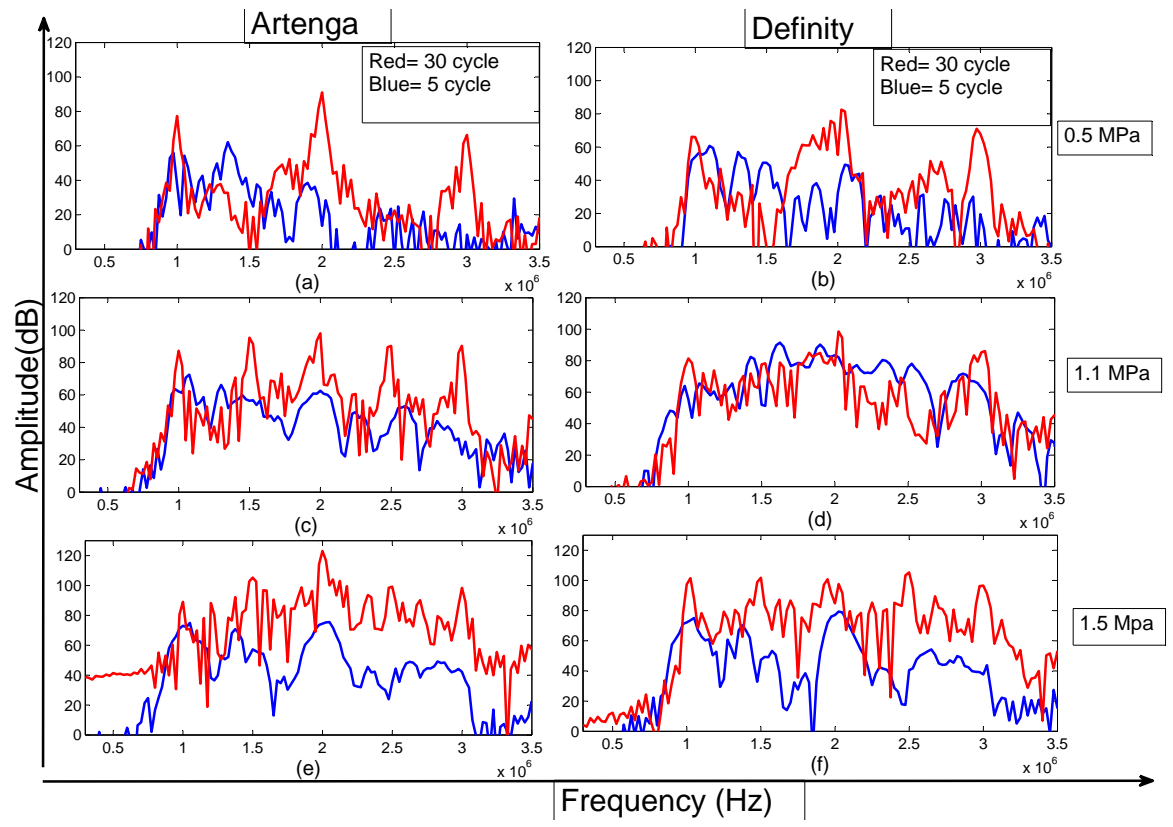

Figure B.6: ART and DEF MBs FFT spectra for 10ms insonation time from second set of experiment as a function of PNP for signals received with $2.25 \mathrm{MHz}$ receiver transducers. Results of two different bubbles are shown for $0.5,1.1,1.5 \mathrm{MPa}$ at 30 and 5 cycle pulse duration. The vertical axis represents amplitude (dB) and horizontal axis represents frequency $(\mathrm{Hz})$. 


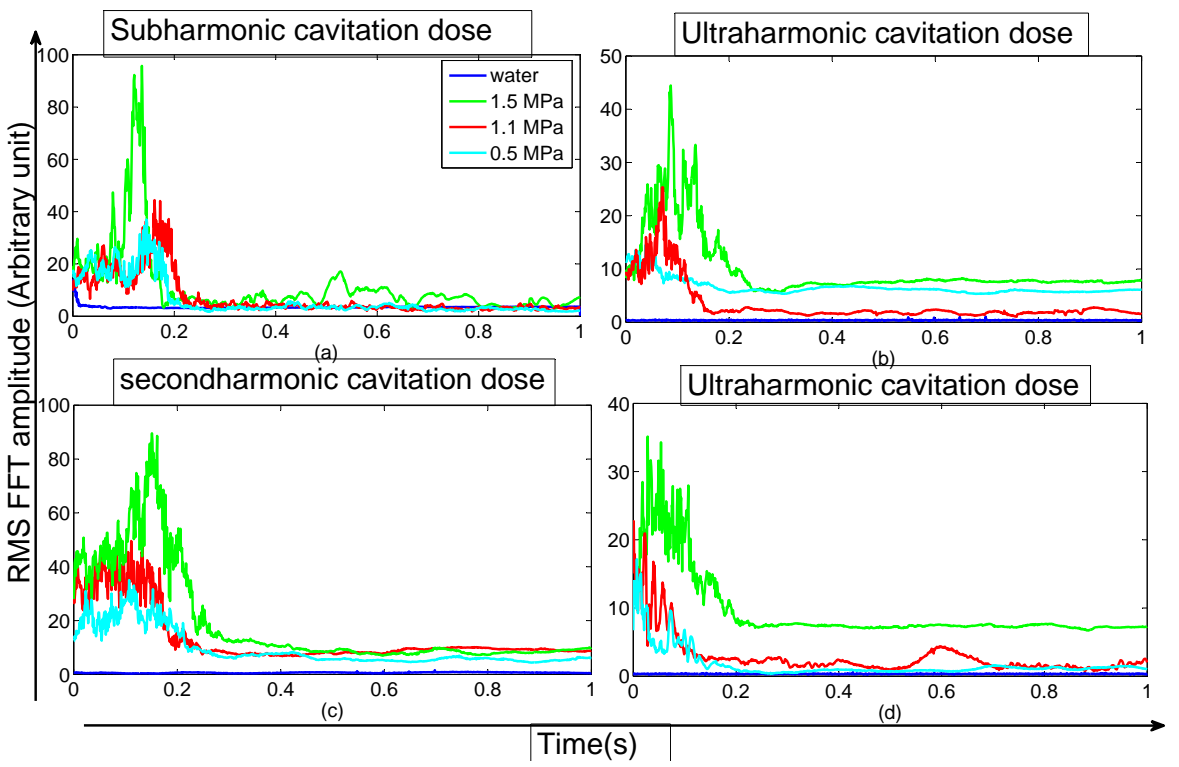

Figure B.7: RMS FFT amplitude vs time plots for DEF MB at 30 cycles pulse duration from signals of experiment 2. (a), (b), ( c) and (d) represent the cavitation dose for subharmonic $(0.5 \pm 0.2 \mathrm{MHz})$, ultraharmonic $(1.5 \pm 0.2 \mathrm{MHz})$, harmonic $(2 \pm 0.2 \mathrm{MHz})$ and ultraharmonic $(2.5 \pm 0.2 \mathrm{MHz})$ respectively.The vertical axis represents the RMS FFT amplitude (arbitrary unit) and horizontal axis represents the time (s).

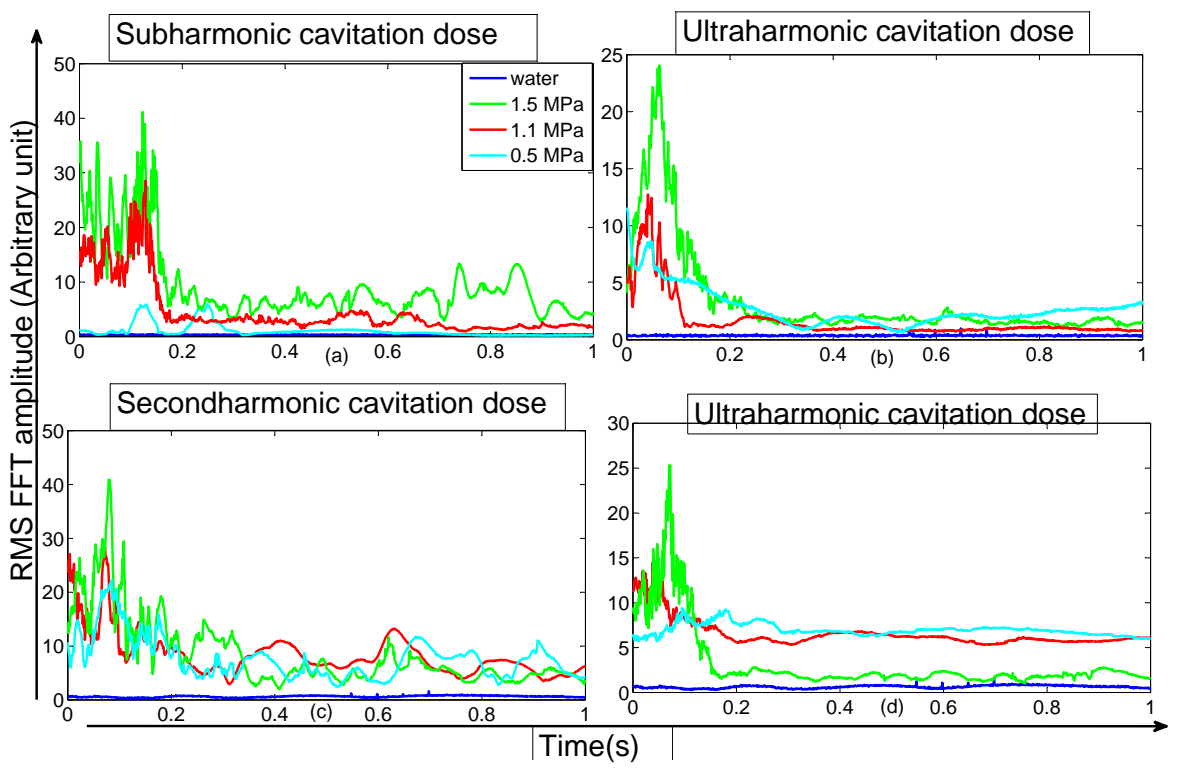

Figure B.8: RMS FFT amplitude vs time plots for ART MB at 30 cycles pulse duration from signals of Experiment 2. (a), (b), ( c) and (d) represent the cavitation dose for subharmonic $(0.5 \pm 0.2 \mathrm{MHz})$, ultraharmonic $(1.5 \pm 0.2 \mathrm{MHz})$, harmonic $(2 \pm 0.2 \mathrm{MHz})$ and ultraharmonic $(2.5 \pm 0.2 \mathrm{MHz})$ respectively.The vertical axis represents the RMS FFT amplitude (arbitrary unit) and horizontal axis represents the time (s). 


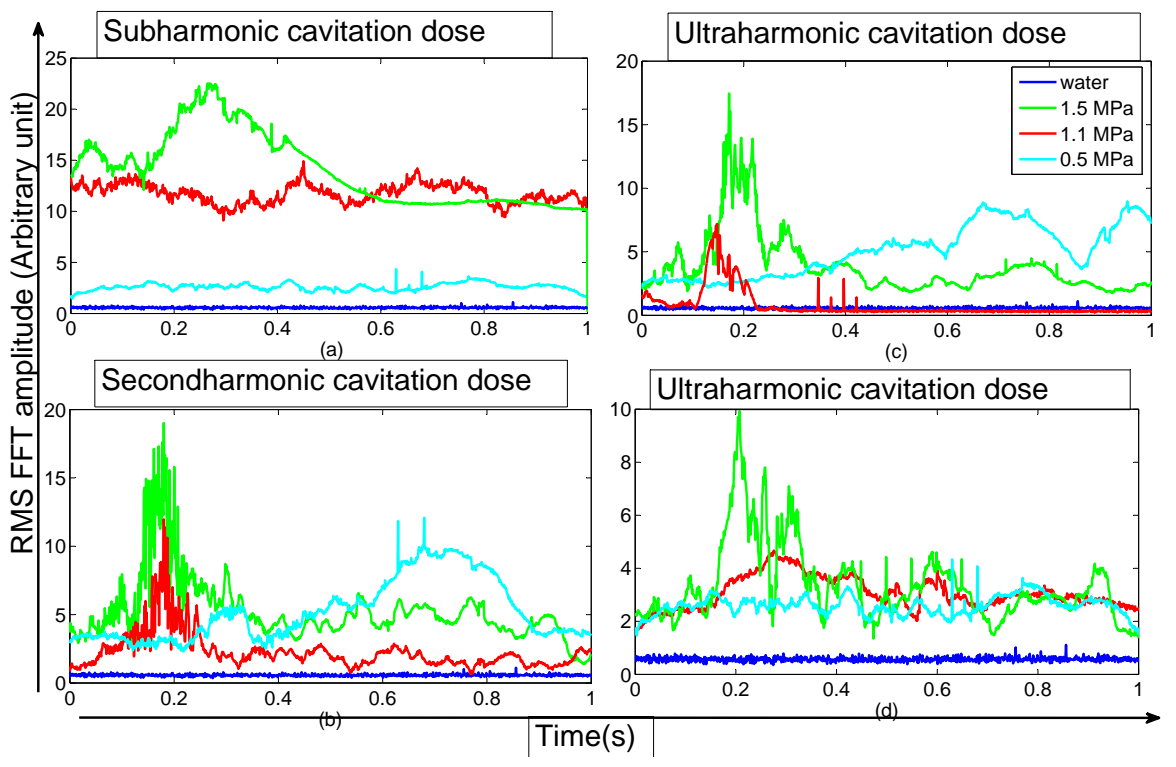

Figure B.9: RMS FFT amplitude vs time plots for DEF MB at 5 cycles pulse duration. (a), (b), (c) and (d) represent the cavitation dose for subharmonic $(0.5 \pm 0.2 \mathrm{MHz})$, ultraharmonic $(1.5 \pm 0.2 \mathrm{MHz})$, harmonic $(2 \pm 0.2 \mathrm{MHz})$ and ultraharmonic $(2.5 \pm 0.2 \mathrm{MHz})$ respectively.The vertical axis represents the RMS FFT amplitude (arbitrary unit) and horizontal axis represents the time (s).

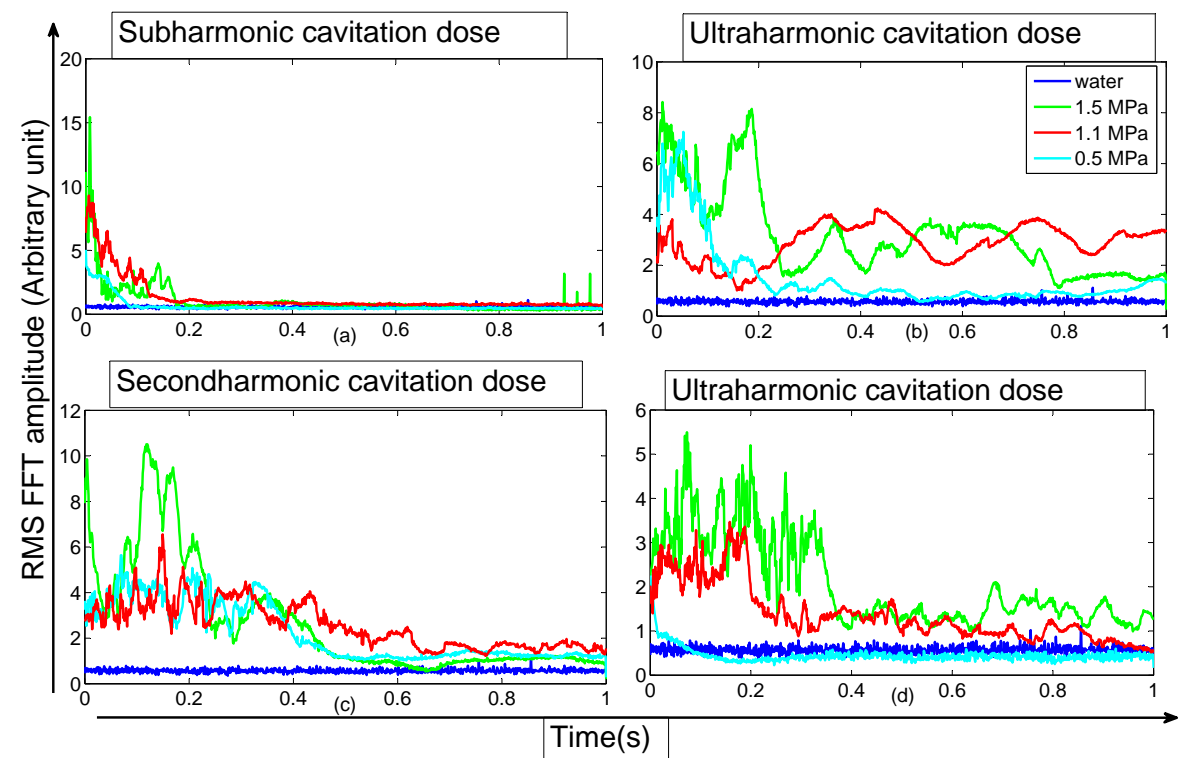

Figure B.10: RMS FFT amplitude vs time plots for ART MB at 5 cycles pulse duration. (a), (b), (c) and (d) represent the cavitation dose for subharmonic $(0.5 \pm 0.2 \mathrm{MHz})$, ultraharmonic $(1.5 \pm 0.2 \mathrm{MHz})$, harmonic $(2 \pm 0.2 \mathrm{MHz})$ and ultraharmonic $(2.5 \pm 0.2 \mathrm{MHz})$ respectively.The vertical axis represents the RMS FFT amplitude (arbitrary unit) and horizontal axis represents the time (s). 


\section{Bibliography}

[1] M Halliwell and Peter N T Wells. A tutorial on ultrasonic physics and imaging techniques. Proceedings of the Institution of Mechanical Engineers, Part H: Journal of Engineering in Medicine, 224(2):127-142, February 2010.

[2] William D. O'Brien Jr. Ultrasound-Biophysicsl Mechanism. Progress in Biophysics and Molecular Biology, 93:212-255, 2007.

[3] Richard S C Cobbold. Foundations of Biomedical Ultrasound. Oxford University Press Inc, 2004.

[4] Nico de Jong, Marcia Emmer, Annemieke van Wamel, and Michel Versluis. Ultrasonic characterization of ultrasound contrast agents. Medical $\& 3$ biological engineering $\mathcal{E}$ computing, 47(8):861-73, August 2009.

[5] H. Uemura, F. Sano, A. Nomiya, T. Yamamoto, M. Nakamura, Y. Miyoshi, K. Miki, K. Noguchi, S. Egawa, Y. Homma, and Y. Kubota. Usefulness of perflubutane microbubble-enhanced ultrasound in imaging and detection of prostate cancer: phase ii multicenter clinical trial. World Journal of Urology, pages 1-6, 2012. cited By (since 1996) 3; Article in Press.

[6] L. J. M. Juffermans, a. Wamel, R. H. Henning, K. Kooiman, M. Emmer, N. Jong, W. H. Gilst, R. Musters, W. J. Paulus, a. C. Rossum, L. E. Deelman, and 
O. Kamp. Ultrasound and microbubble-targeted delivery of therapeutic compounds. Netherlands Heart Journal, 17(2):82-86, February 2009.

[7] Chien-Yu Ting, Ching-Hsiang Fan, Hao-Li Liu, Chiung-Yin Huang, Han-Yi Hsieh, Tzu-Chen Yen, Kuo-Chen Wei, and Chih-Kuang Yeh. Concurrent blood-brain barrier opening and local drug delivery using drug-carrying microbubbles and focused ultrasound for brain glioma treatment. Biomaterials, 33(2):704-12, January 2012 .

[8] Raffi Karshafian, Sanya Samac, Peter D Bevan, and Peter N Burns. Microbubble mediated sonoporation of cells in suspension: clonogenic viability and influence of molecular size on uptake. Ultrasonics, 50(7):691-7, June 2010.

[9] Sophie Hernot and Alexander L Klibanov. Microbubbles in ultrasound-triggered drug and gene delivery. Advanced drug delivery reviews, 60(10):1153-66, June 2008.

[10] J.M. Tsutsui, F. Xie, and R.T. Porter. The use of microbubbles to target drug delivery. Cardiovascular Ultrasound, 2(23):1-7, 2004.

[11] Raffi Karshafian. On the Permeabilisation and Disruption of Cell Membranes by Ultrasound and Microbubbles. Ph.D Thesis, University of Toronto, 2010.

[12] Sophie Mehier-Humbert, Thierry Bettinger, Feng Yan, and Richard H Guy. Plasma membrane poration induced by ultrasound exposure: implication for drug delivery. Journal of controlled release : official journal of the Controlled Release Society, 104(1):213-22, May 2005.

[13] Nahyoung G Lee, Jesse L Berry, Tom C Lee, Annie T Wang, Scott Honowitz, a Linn Murphree, Neeta Varshney, David R Hinton, and Amani a Fawzi. Sonoporation 
enhances chemotherapeutic efficacy in retinoblastoma cells in vitro. Investigative ophthalmology $\&$ visual science, 52(6):3868-73, May 2011.

[14] Chung-Ren Lin, Kuan-Hung Chen, Chien-Hui Yang, Jiin-Tsuey Cheng, Shyr-Ming Sheen-Chen, Chih-Hsien Wu, Wei-Dih Sy, and Yi-Shen Chen. Sonoporationmediated gene transfer into adult rat dorsal root ganglion cells. Journal of biomedical science, 17:44, January 2010.

[15] Raffi Bekeredjian, Helmut F Kuecherer, Richard D Kroll, Hugo a Katus, and Stefan E Hardt. Ultrasound-targeted microbubble destruction augments protein delivery into testes. Urology, 69(2):386-9, February 2007.

[16] Nickolai Sheikov, Nathan McDannold, Natalia Vykhodtseva, Ferenc Jolesz, and Kullervo Hynynen. Cellular mechanisms of the blood-brain barrier opening induced by ultrasound in presence of microbubbles. Ultrasound in medicine 8 biology, 30(7):979-89, July 2004.

[17] Seyedeh Moosavi Nejad, S Hamid R Hosseini, Hidenori Akiyama, and Katsuro Tachibana. Optical observation of cell sonoporation with low intensity ultrasound. Biochemical and biophysical research communications, 413(2):218-23, September 2011.

[18] M.M. Forbes, R.L. Steinberg, and W.D. O'Brien Jr. Frequency-dependent evaluation of the role of definity in producing sonoporatior of chinese hamster ovary cells. Journal of Ultrasound in Medicine, 30(1):61-69, 2011.

[19] Daniel M Hallow, Anuj D Mahajan, Todd E McCutchen, and Mark R Prausnitz. Measurement and correlation of acoustic cavitation with cellular bioeffects. Ultrasound in medicine $\&$ biology, 32(7):1111-22, July 2006. 
[20] Vassilis Sboros. Response of contrast agents to ultrasound. Advanced drug delivery reviews, 60(10):1117-36, June 2008.

[21] Victor F Humphrey. Ultrasound and matter-physical interactions. Progress in biophysics and molecular biology, 93(1-3):195-211, 2007.

[22] Ghaleb A. Husseini William G. Pitt and Bryant J. Staples. Ultrasonic Drug Delivery A General Review. Expert Opin Drug Delivery, 1(1):37-56, November 2006.

[23] Werner Lauterborn and Thomas Kurz. Physics of bubble oscillations. Reports on Progress in Physics, 73(10):106501, October 2010.

[24] W L Nyborg. Ultrasonic microstreaming and related phenomena. The British journal of cancer. Supplement, 5:156-60, March 1982.

[25] Christy K Holland and Robed E Apfel. Thresholds for transient cavitation produced by pulsed ultrasound in a controlled nuclei environment. The Journal of the Acoustical society of America, 88(November):2059-2069, 1990.

[26] A.Y. Ammi, R.O. Cleveland, J. Mamou, G.I. Wang, S.L. Bridal, and W.D. O'Brien Jr. Ultrasonic contrast agent shell rupture detected by inertial cavitation and rebound signals. IEEE Transactions on Ultrasonics, Ferroelectrics, and Frequency Control, 53(1):126-135, 2006.

[27] Ahmet Tezel and Samir Mitragotri. Interactions of inertial cavitation bubbles with stratum corneum lipid bilayers during low-frequency sonophoresis. Biophysical journal, 85(6):3502-12, December 2003.

[28] C.-D. Ohl and B. Wolfrum. Detachment and sonoporation of adherent hela-cells by shock wave-induced cavitation. Biochimica et Biophysica Acta - General Subjects, 1624(1-3):131-138, 2003. 
[29] Philippe Marmottant, Sander van der Meer, Marcia Emmer, Michel Versluis, Nico de Jong, Sascha Hilgenfeldt, and Detlef Lohse. A model for large amplitude oscillations of coated bubbles accounting for buckling and rupture. The Journal of the Acoustical Society of America, 118(6):3499, 2005.

[30] Nico de Jong, Marcia Emmer, Chien Ting Chin, Ayache Bouakaz, Frits Mastik, Detlef Lohse, and Michel Versluis. "Compression-only" behavior of phospholipidcoated contrast bubbles. Ultrasound in medicine $\mathscr{E}$ biology, 33(4):653-6, April 2007.

[31] Shin Yoshizawa, Teiichiro Ikeda, Akira Ito, Ryuhei Ota, Shu Takagi, and Yoichiro Matsumoto. High intensity focused ultrasound lithotripsy with cavitating microbubbles. Medical \& biological engineering \& computing, 47(8):851-60, August 2009 .

[32] Jeroen Slikkerveer, Sebastiaan a Kleijn, Yolande Appelman, Thomas R Porter, Gerrit Veen, Albert C van Rossum, and Otto Kamp. Ultrasound enhanced prehospital thrombolysis using microbubbles infusion in patients with acute ST elevation myocardial infarction: pilot of the Sonolysis study. Ultrasound in medicine $\&$ biology, 38(2):247-52, February 2012.

[33] Saurabh Datta, Constantin-C Coussios, Louis E Mcadory, Jun Tan, Tyrone Porter, Gabrielle De Courten-myers, and Christy K Holland. Correlation of cavitation with ultrasound. Ultrasound in medicine \& biology, 32(8):1257-1267, 2007.

[34] C. C. Coussios, C. H. Farny, G. Ter Haar, and R. A. Roy. Role of acoustic cavitation in the delivery and monitoring of cancer treatment by high-intensity focused ultrasound (HIFU). International Journal of Hyperthermia, 23(2):105-120, January 2007. 
[35] E C Unger, E Hersh, M Vannan, T O Matsunaga, and T McCreery. Local drug and gene delivery through microbubbles. Progress in cardiovascular diseases, 44(1):45-54, 2001.

[36] David E. Goertz, Raffi Karshafian, and Kullervo Hynynen. Investigating the effects of pulsed low intensity ultrasound and microbubbles in mouse tumors. 2009 IEEE International Ultrasonics Symposium, pages 89-92, September 2009.

[37] Katherine Ferrara, Rachel Pollard, and Mark Borden. Ultrasound microbubble contrast agents: fundamentals and application to gene and drug delivery. Annual review of biomedical engineering, 9:415-47, January 2007.

[38] Monica Mary Forbes. The role of ultrasound contrast agents in producing Sonoporation. Ph.D Thesis, University of Illinois at Urbana-Champaign, 2009.

[39] Wen-Shiang Chen, Andrew A. Brayman, Thomas J. Matula, and Lawrence A. Crum. Inertial cavitation dose and hemolysis produced in vitro with or without Optison. Ultrasound in Medicine $\mathscr{E}$ Biology, 29(5):725-737, May 2003.

[40] Nico de Jong, Ayache Bouakaz, and Peter Frinking. Basic acoustic properties of microbubbles. Echocardiography (Mount Kisco, N.Y.), 19(3):229-40, April 2002.

[41] W.T. Shi and F. Forsberg. Ultrasonic characterization of the nonlinear properties of contrast microbubbles. Ultrasound in Medicine and Biology, 26(1):93-104, 2000.

[42] Mathieu Santin, Alexander Haak, Lori Bridal, and William D. O'Brien, Jr. Comparison of Spectral and Temporal Criteria for Inertial Cavitation Collapse. The Journal of the Acoustical Society of America, 123(5):3218, 2008.

[43] Elena Biagi, Luca Breschi, Enrico Vannacci, and Leonardo Masotti. Stable and transient subharmonic emissions from isolated contrast agent microbubbles. IEEE 
transactions on ultrasonics, ferroelectrics, and frequency control, 54(3):480-97, March 2007.

[44] Brandon L Helfield, Emmanuel Cherin, F Stuart Foster, and David E Goertz. Investigating the subharmonic response of individual phospholipid encapsulated microbubbles at high frequencies: a comparative study of five agents. Ultrasound in medicine $\&$ biology, 38(5):846-63, May 2012.

[45] K. Hensel, R. Haagen, G. Schmitz, A. Maghnouj, and S. A. Hahn. Evaluation of subharmonic emission from encapsulated microbubbles as an indicator for sonoporation of cell monolayers. 2009 IEEE International Ultrasonics Symposium, pages 19-22, September 2009.

[46] James McLaughlan, Ian Rivens, Timothy Leighton, and Gail Ter Haar. A study of bubble activity generated in ex vivo tissue by high intensity focused ultrasound. Ultrasound in medicine $\&$ biology, 36(8):1327-44, August 2010.

[47] Ayache Bouakaz, Michel Versluis, and Nico de Jong. High-speed optical observations of contrast agent destruction. Ultrasound in medicine $\mathscr{E}$ biology, 31(3):391-9, March 2005.

[48] Benjamin Dollet, Sander M van der Meer, Valeria Garbin, Nico de Jong, Detlef Lohse, and Michel Versluis. Nonspherical oscillations of ultrasound contrast agent microbubbles. Ultrasound in medicine E biology, 34(9):1465-73, September 2008.

[49] S I Madanshetty, R A Roy, and R E Apfel. Acoustic microcavitation: its active and passive acoustic detection. The Journal of the Acoustical Society of America, 90(3):1515-26, September 1991.

[50] Daniel A. King, Mathieu Santin, Michael J. Malloy, Alayna C. Roberts, Alexander 
Haak, Josquin Foiret, Sylvain Haupert, Sara Jafari, Lori Bridal, and William D. O'Brien. Using passive cavitation detection to observe postexcitation response of ultrasound contrast agents. 2009 IEEE International Ultrasonics Symposium, pages 1286-1289, September 2009.

[51] A Bailey M R Crum Cleveland R, Sapozhnikov O. A dual passive cavitation detector for localized detection of lithotripsy-induced cavitation in vitro. The Journal of the Acoustical Society of America, 107(3):1745-58, March 2000.

[52] Claudio Rota, Carol H. Raeman, Sally Z. Child, and Diane Dalecki. Detection of acoustic cavitation in the heart with microbubble contrast agents in vivo: A mechanism for ultrasound- induced arrhythmias. The Journal of the Acoustical Society of America, 120(5):2958, 2006.

[53] J Hancock, H Dittrich, D E Jewitt, and M J Monaghan. Evaluation of myocardial, hepatic, and renal perfusion in a variety of clinical conditions using an intravenous ultrasound contrast agent (Optison) and second harmonic imaging. Heart (British Cardiac Society), 81(6):636-41, June 1999.

[54] Jeroen Sijl, Benjamin Dollet, Marlies Overvelde, Valeria Garbin, Timo Rozendal, Nico de Jong, Detlef Lohse, and Michel Versluis. Subharmonic behavior of phospholipid-coated ultrasound contrast agent microbubbles. The Journal of the Acoustical Society of America, 128(5):3239-52, November 2010.

[55] M D Santin, D a King, J Foiret, a Haak, W D O'Brien, and S L Bridal. Encapsulated contrast microbubble radial oscillation associated with postexcitation pressure peaks. The Journal of the Acoustical Society of America, 127(2):1156-64, February 2010.

[56] Yuanyuan Qiu, Yi Luo, Yanli Zhang, Weicheng Cui, Dong Zhang, Junru Wu, Jun- 
feng Zhang, and Juan Tu. The correlation between acoustic cavitation and sonoporation involved in ultrasound-mediated DNA transfection with polyethylenimine (PEI) in vitro. Journal of controlled release : official journal of the Controlled Release Society, 145(1):40-8, July 2010.

[57] Chun-Yen Lai, Chia-Hsuan Wu, Chia-Chun Chen, and Pai-Chi Li. Quantitative relations of acoustic inertial cavitation with sonoporation and cell viability. $U l-$ trasound in medicine $\&$ biology, 32(12):1931-41, December 2006.

[58] H. Tang, C.C.J. Wang, D. Blankschtein, and R. Langer. An investigation of the role of cavitation in low-frequency ultrasound-mediated transdermal drug transport. Pharmaceutical Research, 19(8):1160-1169, 2002.

[59] Joo Ha Hwang, Juan Tu, Andrew A. Brayman, Thomas J. Matula, and Awrence A. Crum. Correlation between inertial cavitation dose and endothelial cell damage in vivo. Ultrasound in medicine \& biology, 32(10):1611-1619, 2006.

[60] Juan Tu, Thomas J. Matula, Andrew A. Brayman, and Lawrence A. Crum. Inertial cavitation dose produced in ex vivo rabbit ear arteries with optison by $1-\mathrm{MHz}$ pulsed ultrasound. Ultrasound in Medicine $\&$ Biology, 32(2):281-288, February 2006.

[61] Jameel A Feshitan, Cherry C Chen, James J Kwan, and Mark A Borden. Microbubble size isolation by differential centrifugation. Journal of colloid and interface science, 329(2):316-24, January 2009.

[62] G. Renaud, J. G. Bosch, A. F. W. van der Steen, and N. de Jong. An acoustical camera for in vitro characterization of contrast agent microbubble vibrations. Applied Physics Letters, 100(10):101911, 2012. 